\title{
Characterization of neuroligin 4, a protein involved in autism spectrum disorders
}

\author{
Dissertation \\ for the award of the degree \\ Doctor rerum naturalium (Dr. rer. nat.)
}

Division of Mathematics and Natural Sciences

of the Georg-August-Universität Göttingen

\author{
submitted by \\ Matthieu Hammer \\ from Haguenau, France
}

Göttingen, May 2012 
Thesis committee members

Prof. Dr. Nils Brose (reviewer)

Molecular Neurobiology, Max Planck Institute for Experimental Medicine

\section{Dr. Oliver Schlüter (reviewer)}

Molecular Neurobiology, European Neurosciences Institute Göttingen

\section{Prof. Dr. Swen Hülsmann}

Neuro- and Sensory Physiology, University of Göttingen

\section{Extended committee members}

Dr. Camin Dean

Trans-synaptic Signalling, European Neuroscience Institute Göttingen

\section{Prof. Dr. Siegrid Löwel}

System Neurosciences, University of Göttingen

\section{Prof. Dr. Jochen Staiger}

Neuroanatomy, University of Göttingen

Date of the oral examination: June 13th, 2012 


\section{Declaration}

I hereby declare that this thesis "Characterization of neuroligin 4, a protein involved in autism spectrum disorders" has been written independently, with no other help than those quoted.

Matthieu Hammer 
"La science n'a pas de patrie, parce que le savoir est le patrimoine de l'humanité." Louis Pasteur 


\section{Acknowledgements}

I would like to express my thanks to my supervisors Dr. Frédérique Varoqueaux and Prof. Nils Brose for having integrated me as $\mathrm{PhD}$ student in this lab and having supervised me throughout these years in a great scientific atmosphere letting me develop my scientific skills throughout the world of synapses.

I thank Dr. Oliver Schlüter and Prof. Dr. Swen Hülsmann who, as members of my thesis committee, provided input and reflection on the progress of my work.

I am very thankful to the additional committee members, Dr. Camin Dean, Prof. Dr. Siegrid Löwel and Prof. Dr. Jochen Staiger for evaluating my thesis work.

I would like to thank my $\mathrm{PhD}$ program, the CMPB. I was honoured to be the representative student from this program during one year and it gave me great pleasure to intensively interact with Prof. Dr. Michael Hörner. I thank the Göttingen graduate school GGNB to provide such a good scientific training and atmosphere to us.

All this work is also the result of a lot of interaction and collaboration with the members of the Molecular Neurobiology Department. It was an honour working with Dilja, Ben, Cordelia, Liam, Christoph, Marylin, Fritz, Klaus, Martin, Anja, Alex, Tolga and Tina. I would like to express my special gratefulness to: Dilja and Liam. We formed an extraordinary neuroligin team that through the last two years worked in a professional and at the same time supportive, helpful and enjoyable atmosphere and this work is the result of a lot of passionate discussion and support. I would also like to express my special gratefulness to Ben, for his limitless patience and to introduce me to the fabulous world of imagery; and Christoph for giving me the opportunity to make my first steps in the field of electrophysiology. 
The scientific community present in Göttingen gave me the opportunity to meet so many wonderful people, some of which would ultimately become my friends and were always present for the best and the worst times that every graduate student has to go through. I would like to thank Dilja and Liam for being more than just lab colleagues, but very good friends. I thank Anneuh, Stéphanie, Andrea, Derya, Alwaleed, Sanaz, Natalia, Jonas, Andrew and many more... for the wonderful "Leute group" that we made together and giving us the opportunity to learn a lot from each other and for all the discussions that we had all together.

Je tiens également à remercier ceux que j'ai quittés pour poursuivre mes études à Göttingen. Katuschia et Vincent, un grand merci pour toutes ces années d'amitiés sans failles que nous vivons depuis de nombreuses années. Même avec la distance, nous avons réussi à garder cette précieuse complicité.

Et puis il y a ma famille, tout d'abord je tiens à embrasser ma petite nièce Ava qui a vu le jour pendant ma thèse et qui reste au centre de toute les attentions et discussion depuis. C'est un veritable rayon de soleil qui nous illumine. Je tiens également à remercier ma soeur Sandrine, elle qui a été toujours une source d'inspiration, un guide et d'une précieuse aide à chaque étape de ma vie.

Maman, Papa, sans vous, sans votre soutien rien de tout ça n'aurait été possible. Merci pour votre support inconditionnel durant ces années d'études, je sais que ca n'a pas toujours été facile et pourtant vous avez été toujours présent à mes côtés. 


\section{Table of Contents}

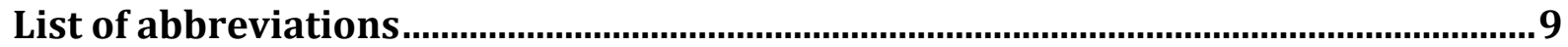

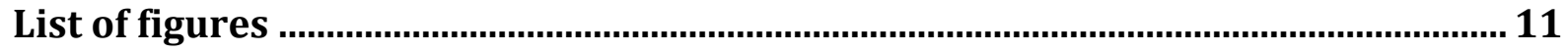

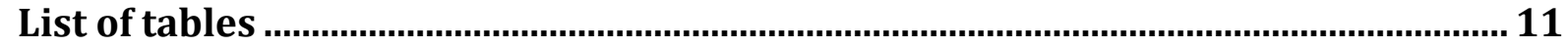

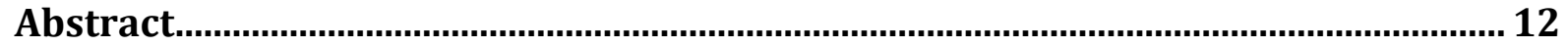

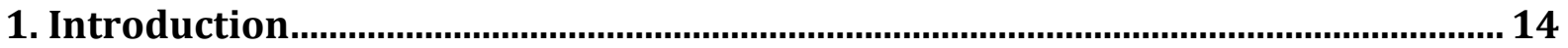

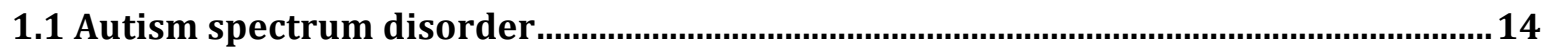

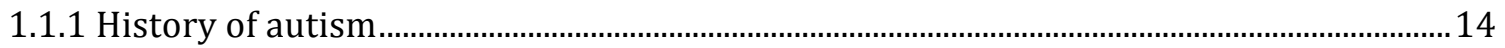

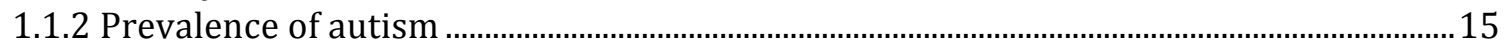

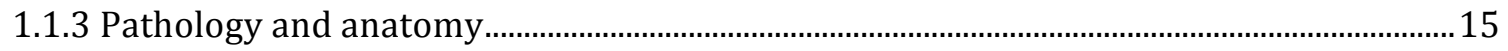

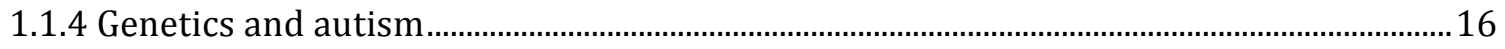

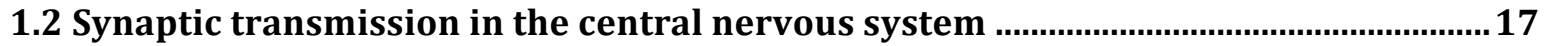

1.2.1 Neurons communicate through synapses...........................................................................

1.2.2 Excitatory and inhibitory postsynaptic architecture …….................................................... 19

1.2.3 Synaptogenesis and synaptic maturation …………................................................................. 20

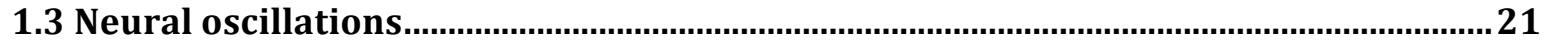

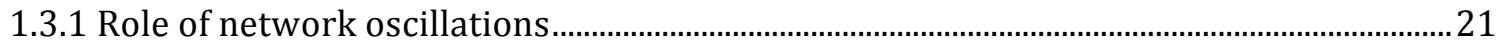

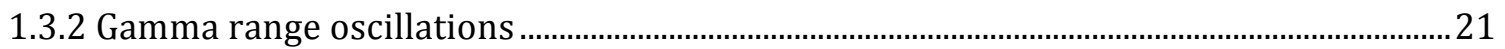

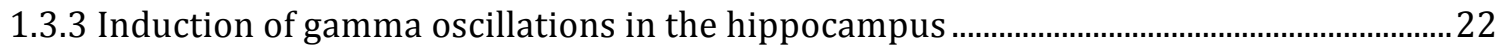

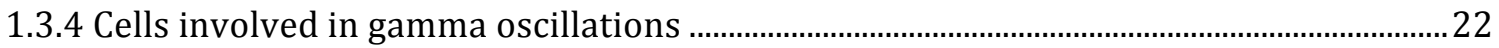

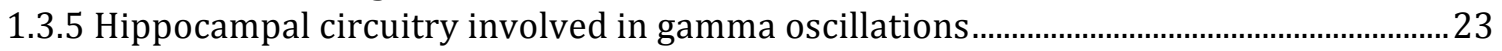

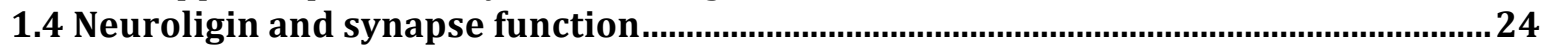

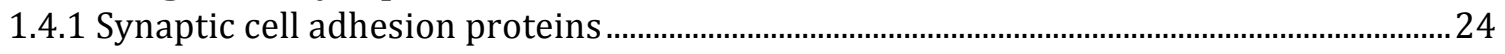

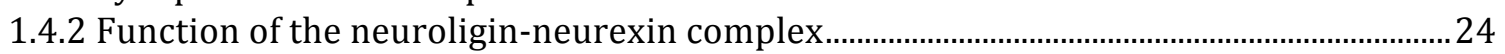

1.4.3 Role of neuroligins at mature synapses …………….......................................................... 30

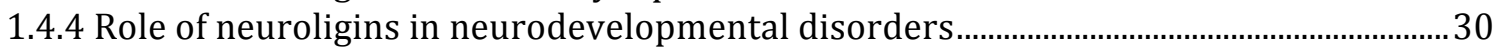

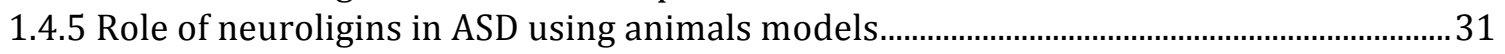

1.5 Aim of the present study

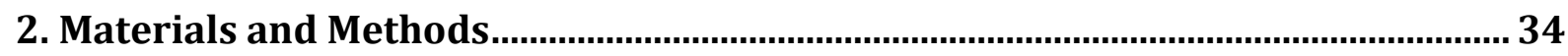

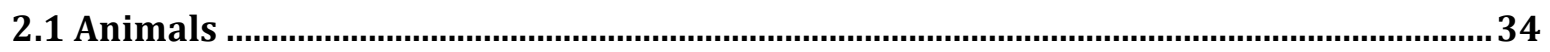

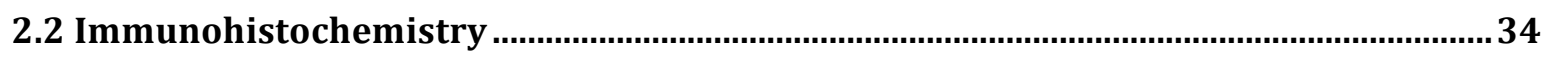

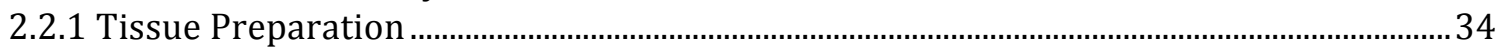

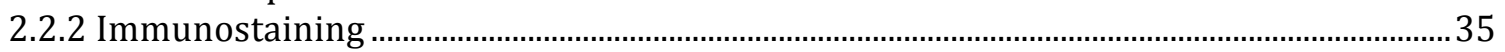

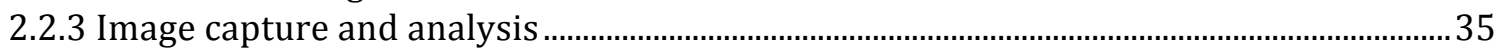

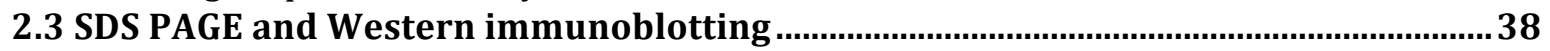

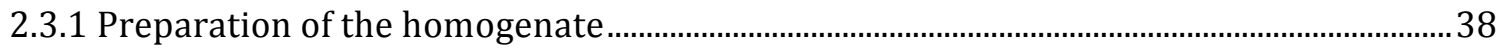

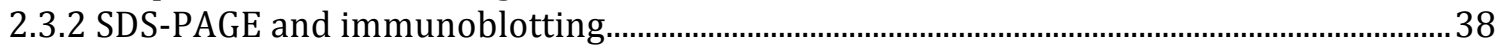

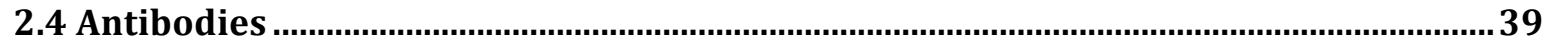

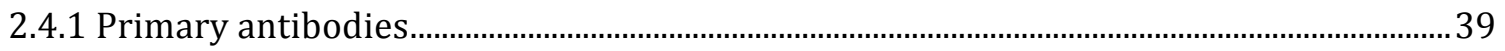

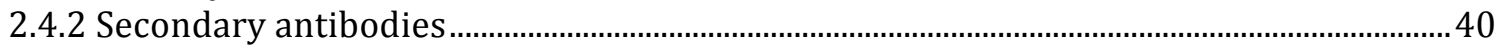

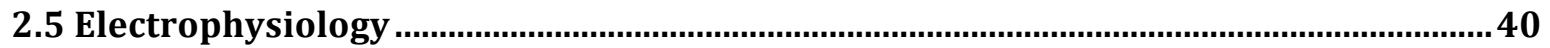

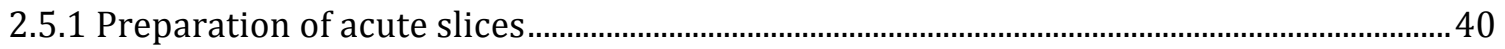

2.5.2 Gamma oscillations under interface conditions ....................................................................... 41

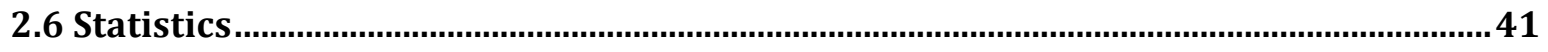

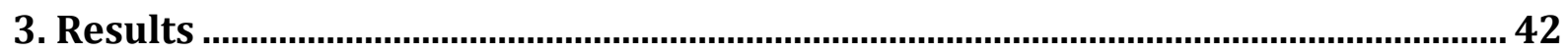

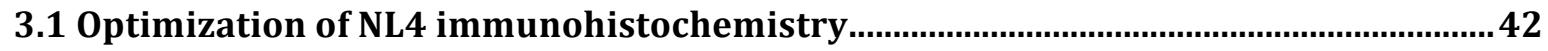

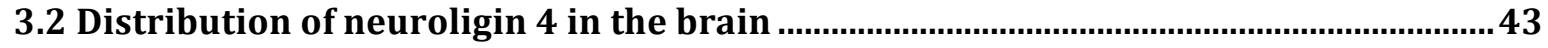

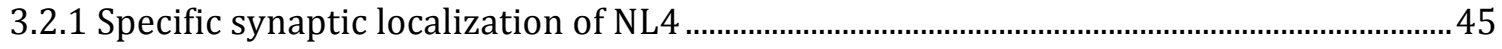

3.2.2 Characterization of NL4-containing synapses ……......................................................... 49 
3.2.2.1 NL4 is present at inhibitory synapses in the globus pallidus and brainstem ..........................50

3.2.2.2 NL4 is present at excitatory synapses in the barrel cortex ........................................................55

3.2.2.3 NL4 is present at excitatory and inhibitory synapses in the hippocampus ...............................57

3.3 Characterization of NL4 deletion mutant mice...........................................................59

3.3.1 NL4 knockout mice show no alteration in synaptic circuitry.............................................59

3.3.2 Upregulation of neuroligins isoforms as a consequence of the NL4 knockout...............62

3.3.3 NL4-KO mice have strongly reduced gamma oscillations in CA3 ......................................63

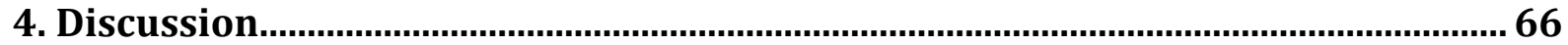

4.1 Characterization of NL4 distribution by immunohistochemistry ..................................67

4.1.1 Methodological considerations for NL4 immunohistochemistry .....................................67

4.1.2 Distribution of neuroligin 4 in regions of the central nervous system that are relevant

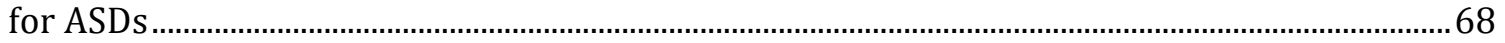

4.1.3 Correlation between localization of NL4 protein and mRNA ............................................. 72

4.2 Postsynaptic architecture of NL4 positive synapses ................................................ 73

4.2.1 Global immunoreactivity pattern of NL4 in relationship to gephyrin and PSD95 ........74

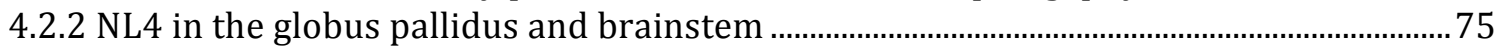

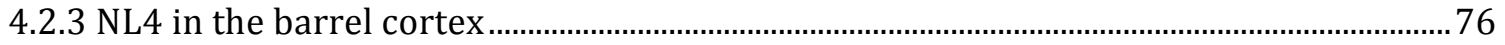

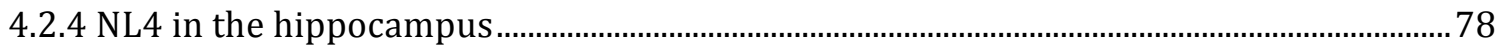

4.3 Effect of the loss of NL4 on synapse number and distribution ..................................... 79

4.3.1 Gephyrin and PSD-95 containing synapses are unchanged in the hippocampus and the barrel cortex in NL4-KO mice .................................................................................................... 79

4.3.2 Unchanged number of glycine receptor containing synapses in the spinal cord of NL4-

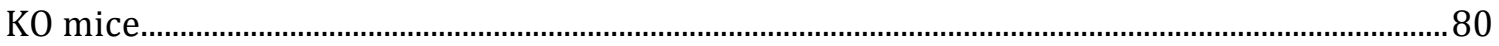

4.3.3 Functional redundancy of NLs isoforms in NL4-KO mice ..................................................81

4.4 Decreased gamma oscillations in NL4-KO mice ............................................................8 82

5. Summary and Conclusions................................................................................ 86

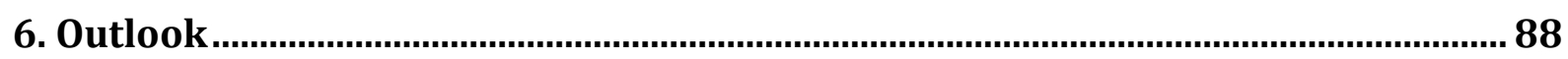

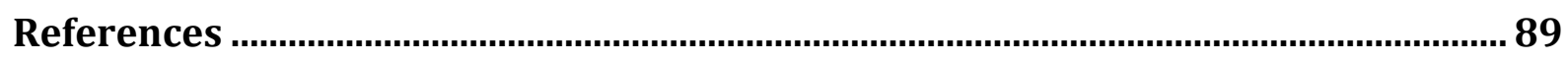

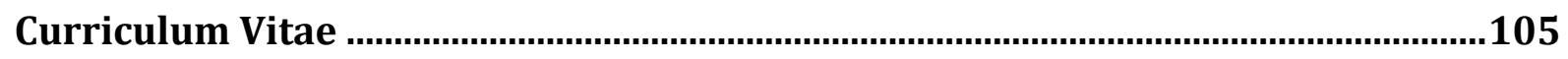

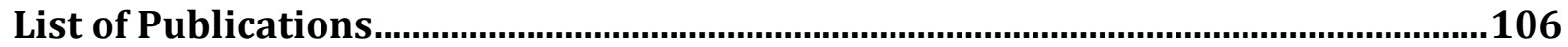




\section{List of abbreviations}

\begin{tabular}{|l|l|}
\hline ACSF & artificial cerebral spinal fluid \\
\hline ADDM & Autism and Developmental Disabilities Monitoring \\
\hline AMPA & $\alpha$-amino-3-hydroxy-5-methyl-4-isoxazolepropionic acid \\
\hline ASD & autism-spectrum-disorder \\
\hline CA & Cornu Ammonis \\
\hline CASK & calcium/calmodulin-dependent serine protein kinase \\
\hline CCK & cholecystokinin \\
\hline CDC & Centers for Disease Control and Prevention \\
\hline CNS & central nervous system \\
\hline DHPG & ( (s)-3,5-dihydroxyphenylglycine \\
\hline E/I & excitatory/inhibitory \\
\hline EGF & epidermal growth factor \\
\hline EPSCs & spontaneous excitatory postsynaptic currents \\
\hline EPSP & excitatory postsynaptic potential \\
\hline fMRI & functional magnetic resonance imaging \\
\hline GABA & $\gamma$-aminobutyric acid \\
\hline IHC & immunohistochemistry \\
\hline IPSP & inhibitory postsynaptic potential \\
\hline KO & knock out \\
\hline LGP & lateral globus pallidus \\
\hline LNS & laminin, NX, sex-hormone-binding protein \\
\hline LRRTMs & leucine-rich repeat transmembrane proteins \\
\hline mAChRs & muscarinic acetylcholine receptors \\
\hline mGluRs & metabotropic glutamate receptors \\
\hline NL & neuroligin \\
\hline NL4-KO & neuroligin 4 knockout \\
\hline NMDA & N-methyl-D-aspartic acid \\
\hline NX & neurexin \\
\hline PBS & phosphate buffered saline \\
\hline PDZ & PSD-95/disc large/zona-occludens-1 \\
\hline PFA & paraformaldehyde \\
\hline PSD-95 & postsynaptic density protein of 95 kDa \\
\hline PSDs & postsynaptic densities \\
\hline PV & parvalbumin \\
\hline REM & rapid eye movement \\
\hline Rt & reticular thalamic nucleus \\
\hline S-SCAM & synaptic scaffolding molecule \\
\hline SNR & substantia nigra \\
\hline str. l-m & stratum lacunosum moleculare \\
\hline str. or & stratum oriens \\
\hline str.pyr & stratum pyramidale \\
\hline str. rad & stratum radiatum \\
\hline TARPs & Transmembrane AMPA regulatory proteins \\
\hline VGluT & vesicular glutamate transporter \\
\hline
\end{tabular}




\begin{tabular}{|l|l|}
\hline VIAAT & vesicular inhibitory amino acid transporter \\
\hline VP & ventral pallidum \\
\hline VPL & ventral posterior nucleus \\
\hline WT & wild type \\
\hline$\alpha 7$ nAChRs & $\alpha 7$ nicotinic acetylcholine receptor subunit \\
\hline
\end{tabular}




\section{List of figures}

Figure 1.1: Synaptic organization of neurexin neuroligin interactions

Figure 1.2: Schematic diagram of the anatomical connectivity with the neuroligins isoforms

Figure 2.1: Quantification of puncta numbers and percentage of colocalization between two synaptic markers

Figure 3.1: Immunoreactivity of neuroligin 4 in the brain and the spinal cord

Figure 3.2: NL4 distribution in coronal brain sections

Figure 3.3: NL4 localization matches gephyrin labeling

Figure 3.4: NL4 is associated with excitatory and inhibitory synapses depending on the brain region

Figure 3.5: Localization of NL4 in the globus pallidus

Figure 3.6: Localization of NL4 in the brainstem

Figure 3.7: NL4 is associated with $\mathrm{GABA}_{\mathrm{A}} \mathrm{R} \gamma 2$ and GlyR $\alpha 1$ receptor subunits in brainstem

Figure 3.8: Localization of NL4 in the barrel cortex

Figure 3.9: Localization of NL4 in the hippocampus (CA1 stratum lacunosum moleculare)

Figure 3.10: No alterations in excitatory and inhibitory synapse density in the NL4-KO barrel cortex or hippocampus

Figure 3.11: No alterations in glycinergic synapse density in the NL4-KO spinal cord

Figure 3.12: Upregulation of NL2 and NL3 in the absence of NL4

Figure 3.13: Reduced hippocampal gamma oscillations in NL4 knockout mice

\section{List of tables}

Table 2.1: Primary antibodies used for this project

Table 2.2: Secondary antibodies used in this study 


\section{Abstract}

Neuroligins (NLs) are ubiquitous postsynaptic adhesion proteins that are essential for synaptic maturation and function. Of the four NL isoforms expressed in the central nervous system (CNS), NL4 is of particular interest since loss-of-function mutations of NL4 have been associated with autism-related disorders in humans (Jamain et al., 2003). Furthermore, NL4 knockout mice were found to show specific behavioral phenotypes that mimic those observed in autism (Jamain et al., 2008). In the present study, the distribution of NL4 protein was characterized in the mouse central nervous system (CNS) using a NL4 specific antibody.

The highest NL4 protein expression levels were observed in brainstem, spinal cord and globus pallidus. At the synaptic level, NL4 is localized to a majority of inhibitory postsynapses in the globus pallidus, brainstem and spinal cord, and to glycinergic synapses in the brainstem and spinal cord (Hoon et al., 2011). Interestingly, NL4 is not exclusively restricted to inhibitory postsynapses in the forebrain. In the hippocampus, NL4 equips both inhibitory and excitatory synapse types, whereas in the barrel cortex NL4 seems to be preferentially associated with excitatory synapses.

In order to study the role of NL4 at synapses, the consequences of NL4 deletion on synapse number was examined. In the barrel cortex and hippocampus, the loss of NL4 did not influence the density of excitatory or inhibitory synapses. Similarly, no differences were observed in the number of glycinergic synapses in the spinal cord. The absence of an effect of NL4-loss on the number of synapses in the CNS could potentially be explained by a compensatory effect of one of the other NL isoforms. In line with this hypothesis, an upregulation of NL2 and NL3 levels was observed in the somatosensory cortex of the NL4$\mathrm{KO}$ mice.

Interestingly, in the absence of NL4, the power of kainate-induced gamma oscillations in area CA3 of the hippocampus was found to be substantially reduced. This phenotype indicates a defect in the synaptic network between pyramidal cells and inhibitory interneurons 
in the hippocampus. Together, these data provide new insights into the physiological consequences of NL4 loss in autism spectrum disorders, which may lead to an imbalance of excitatory and inhibitory synaptic transmission in various brains regions, including the hippocampus. 


\section{Introduction}

\subsection{Autism spectrum disorder}

\subsubsection{History of autism}

Autism was first described during the first half of the twentieth century. Physicians working on child developmental abnormalities tried to classify the different child psychoses. One of these physicians, Leo Kanner, outlined the classic description of a so-called "early infantile autism" (Kanner, 1943). He described eight-year-old children who were socially impaired, mute and had automatic repetition of vocalization. In addition, these children were intensively resistant to change in their own repetitive routines. One year later, Hans Asperger described older children who were naïve and inappropriate in their social interaction, had good speech but used it for monologues on their own special interests and were absorbed in their circumscribed interests. These children were close to being considered normal or superiorly intelligent, but often had significant difficulties in specific learning tasks. Asperger used the term "autistic" to describe the behavior he saw (Asperger, 1944). Approximately twenty years later, these two types of "autism" were amalgamated into the same group of neurodevelopmental disorders based on their overlapping symptoms.

Despite the extensive description of disease symptoms, no physical pathology was observed in the brain of these children. Kanner therefore suggested that the children were initially normal from a pathological point of view, but that they became emotionally damaged due to parental detachment, lack of humor and strictness. Thus, autism became classified as a psychiatric disorder that was caused by environmental factors during the development of the child.

It was not until the 1980's that autism became classified as a neurodevelopmental disorder that may originate from or be facilitated by physiological dysfunctions. Today, the descriptions of Kanner and Asperger have been greatly expanded upon, and autism is considered to be composed of a wide spectrum of overlapping and non-overlapping symptoms. "The Diagnostic and Statistical Manual of Mental Disorders, fourth edition" 
defined autism-spectrum-disorder (ASD) as a neurodevelopmental disorder that is diagnosed based on three main symptoms that are present at varying degrees of intensity:

(1) An impairment in social interaction skills, (2) a lack of appropriate language and communication skills and (3) repetitive behavior and lack of imagination.

In other words, autism is not a single entity but a combination of clinical symptoms. In addition to the three key elements of ASD as mentioned above, other clinical dysfunctions can be observed, such as sensory and motor abnormalities, gastrointestinal dysfunction, and sleep disturbance.

\subsubsection{Prevalence of autism}

About 1/88 children has been identified with ASDs in the USA according to the Centers for Disease Control and Prevention (CDC) and the Developmental Disabilities Monitoring (ADDM) network in 2008 (Baio, 2012). The prevalence of ASDs has been increasing worldwide for unknown reasons. One possible explanation is that reporting of the disorder has risen due to increasing awareness and an extension of clinical symptoms belonging to the ASDs. Diagnosis of ASDs, as with many other psychiatric diseases, is dependent on observations of behavior and cognition that can sometimes be subjective. Similarly, clinical manifestations such as cognitive impairments can differ widely between children suffering from the same disorder. This variability may lead to greatly varying numbers of diagnoses depending on observer awareness. In addition, it has been suggested that the increased prevalence could be due to modern day living practices. For example, it has been reported that environmental toxins or parental age were associated with autism (Kolevzon et al., 2007; Herbert, 2010; Landrigan, 2010), however there is currently insufficient evidence to support this notion. Interestingly, the prevalence also differs across the sexes. According to the last CDC report, one in 54 boys and one in 252 girls were identified as having an ASD (Baio, 2012).

\subsubsection{Pathology and anatomy}

Brain development seems to be affected in ASD patients, especially brain volume (Courchesne et al., 2003; Hazlett et al., 2005). An increase in brain volume was observed in the frontal lobes and the anterior temporal regions (Courchesne et al., 2007). One theory 
proposes that in autism, short-range connections are increased, whereas longer-range connections are reduced, as it has been observed that the volume of the corpus callosum is decreased (Just et al., 2006). In terms of cellular abnormalities, some studies showed a disruption of the cortical layer in the frontal lobe and a disorganization of the mini columnar organization (Buxhoeveden et al., 2006). Furthermore, a decrease in the number of neurons was observed in the amygdala, a region believed to be involved in emotion (Schumann and Amaral, 2006).

\subsubsection{Genetics and autism}

Epidemiologic studies provide evidence for a genetic basis of ASDs. In the past ten years, there has been an explosion of new potential gene candidates linked to ASDs that are helping to understand the genetic components that contribute to the physiopathology of the disease. Although the underlying genetic architecture of ASDs is still not well known, the literature demonstrates that it is not a monogenic disorder with Mendelian inheritance, but rather a group of complex genetic syndromes with risk deriving from genetic variations in multiple genes, although in some rare cases, single gene mutations have been correlated with ASDs. Interestingly, a noticeable correlation was observed between genes involved in ASDs and those that code for synaptic proteins. A 1999 study on patients with ASD highlighted de novo deletions of the Xp22 region on the $\mathrm{X}$ chromosome (Thomas et al., 1999), a region including the neuroligin $4 \mathrm{X}$ gene. The synaptic protein neuroligin and its binding partner neurexin function as synaptic cell adhesion molecules that also interact with intracellular proteins. Mutations in neurexin or neuroligin function lead to cognitive disorders such as ASDs (described in detail in section 1.4.4). Other synaptic candidates were observed in patients and some familial cases of ASDs, such as neurexins or SHANK (described in section 1.4.3). These studies demonstrate a strong link between specific cases of ASD and proteins involved in synaptic function. The second pathway of genes associated with ASDs are genes encoding proteins that play a role in regulating synaptic protein synthesis, such as TSC1/TSC2, NF1, FMRP or PTEN. Mutations in these proteins lead to syndromic disorders associated with ASDs, including tuberous sclerosis, neurofibromatosis, fragile X syndrome or ASD with macrocephaly (Dölen and Bear, 2008). These studies emphasized an involvement of the synaptic architecture and activity in ASDs. The fact that loss or dysfunction of these proteins in the brain can be directly associated with neurological disorders emphasizes the 
importance of synaptic integrity in the brain development process, especially during early brain development.

\subsection{Synaptic transmission in the central nervous system}

\subsubsection{Neurons communicate through synapses}

The human brain is capable of higher cognitive processes like memory, emotions, performance of precise and highly controlled movements and logical reasoning. There are mainly two types of cells in the brain, neurons and glial cells. Due to their physiological properties, neurons can generate electrical signals, known as action potentials, and these signals can be propagated to others cells via contact points known as synapses ffrom the Greek "syn-“ ("together") and "haptein" ("to clasp"), described by Sir Charles Scott Sherrington in 1897\}. Hundreds of billions of neurons are estimated to be interconnected by a 100 trillion synapses in the human brain. The degree of complexity present in the brain requires the correct development and wiring of all of these neurons in the central nervous system. Any perturbation in brain integrity can lead to severe functional impairment. Indeed, from the earliest age of the development until death, neurons are programmed to make connections with others cells from short to really long distances. Any alteration of the connectivity can lead to neurodevelopmental disorders such as autism, epilepsy or schizophrenia.

The function of synapses is to convert the presynaptic action potential into a signal that the postsynaptic neuron or muscle fiber can identify. Based on the mechanism by which this signal is transmitted, synapses are classified into two groups, chemical and electrical. Electrical synapses consist of a mechanical and conductive junction between two neurons. The electric signal is not transformed into a chemical signal as in the chemical synapse, but travels from one cell to the other without processing by a narrow gap called the gap junction. The chemical synapse is an asymmetrical cell contact area separated by a $20 \mathrm{~nm}$ wide synaptic cleft. Signal transmission across these synapses is a unidirectional process, during which an electrical signal arriving at the presynaptic terminal is converted into a chemical signal or neurotransmitter, which then diffuses across the synaptic cleft to the postsynaptic side, where it is converted back into an electrical signal. The pre-synaptic and the post- 
synaptic sides are each characterized by specific protein complexes that are required for their particular function. Since this process and the accompanying protein complexes are central to the experiments conducted in this thesis, they are described in more detail below.

The axonal electrical stimulation arrives in the presynaptic side, the active zone, where the vesicles containing the neurotransmitters are present (Couteaux and Pécot-Dechavassine, 1973; Landis, 1988). This induces the opening of voltage-gated calcium channels, and the resulting calcium influx triggers the exocytosis of vesicles containing neurotransmitters at the presynaptic membrane (Sudhof, 2004). Neurotransmitter exocytosis requires specific mechanisms and proteins involved in the vesicle release process and its regulation (Wojcik and Brose, 2007). Neurotransmitters are commonly described as "Excitatory", "Inhibitory" or modulatory, depending on the outcome they trigger at the postsynaptic membrane. Glutamate is the main excitatory neurotransmitter, while there are two main inhibitory neurotransmitters, $\gamma$-aminobutyric acid (GABA) and Glycine. At excitatory synapses, the vesicles that are filled with glutamate contain the specific vesicular glutamate transporter (VGluT) (reviewed in Takamori, 2006) and at inhibitory synapses, the vesicular inhibitory amino acid transporter (VIAAT) transports GABA and Glycine (Sagné et al., 1997; Wojcik et al., 2006).

The released neurotransmitters diffuse through the synaptic cleft, and bind to specific receptors on the postsynaptic side. The postsynaptic side is electron-dense and composed of clustered receptors associated with cytoplasmic proteins scaffolding proteins, and cell adhesion proteins such as neuroligins (NLs) (Sheng and Hoogenraad, 2007). The binding of neurotransmitter activates the receptors by modifying their conformation. The response induced by the GABA or glutamate release at the synaptic cleft is processed at the postsynaptic membrane by the ionotropic receptors (involving a transmembrane ion channel) for the fast response and/or by the metabotropic receptors (involving a series of intracellular events) for the modulatory response. The activation of ionotropic receptors (ligand-gated ion channels) by glutamate or GABA determines if the synaptic response is excitatory or inhibitory, respectively. At excitatory synapses, the activation of ionotropic receptors by glutamate triggers a cation influx, leading to a membrane depolarization known as an excitatory postsynaptic potential (EPSP). At inhibitory synapse, a chloride influx triggered in response to the binding of GABA causes a membrane hyperpolarization known as an inhibitory postsynaptic potential (IPSP) (Fritschy and Brünig, 2003; Mody and Pearce, 2004; 
Okabe, 2007). In addition, neurotransmitters can bind to metabotropic (G-protein coupled) receptors, which then activate a series of intracellular signaling cascades. These cascades can then for example indirectly result in the opening of an ion channel via a chemical second messenger, the release of calcium from intracellular stores, and many other effects.

\subsubsection{Excitatory and inhibitory postsynaptic architecture}

The ultrastructure of excitatory and inhibitory synapses differs substantially, such that they can be identified by electron microscopy using a classification according to Gray (Gray, 1959). Type 1 synapses (excitatory) are asymmetric with an extended postsynaptic electron dense domain. Type 2 synapses (inhibitory) are symmetric and do not present this postsynaptic electron dense domain.

Excitatory postsynaptic densities (PSDs) contain two types of ionotropic glutamate receptors, NMDA (N-methyl-D-aspartic acid) and AMPA ( $\alpha$-amino-3-hydroxy-5-methyl-4isoxazolepropionic acid) receptors, named after the specific chemical agonist that they are activated by (Moon et al., 1994; Okabe, 2007). AMPA receptors are composed of four types of subunits, GluA1, GluA2, GluA3, GluA4, that combine to form a tetramer. NMDA receptors are composed of three subunits GluN1, GluN2, GluN3, and form a heterotetramer between two obligatory GluN1 subunits and two GluN2 subunits. Transmembrane AMPA regulatory proteins (TARPs) that have been shown to interact with AMPA receptors are also highly concentrated in the PSDs. Stargazin, one of the members of the TARP family, is responsible for the clustering and the regulation of AMPA receptors at the postsynaptic membrane (Chen et al., 2000). Furthermore, the PSD contains scaffolding proteins such as the postsynaptic density protein of $95 \mathrm{kDa}$ (PSD-95). PSD-95 is composed of PSD-95/disc large/zona-occludens-1 (PDZ) domains followed by an src homology (SH3) and a guanylate kinase domain (reviewed in Kim and Sheng, 2004). The first two PDZ domains mediate the interaction with NMDA receptors and voltage-gated potassium channels, and the third domain can interact with members of the neuroligin family of synaptic adhesion molecules (Kornau et al., 1995; Kim et al., 1995a; Irie et al., 1997).

The inhibitory postsynaptic ultrastructure is composed of the ionotropic $\mathrm{GABA}_{\mathrm{A}}$ and glycine receptors that form chloride-permeable channels and are both heteropentamers. In 
mature neurons, the activation of the chloride channel produces a hyperpolarization of the postsynaptic membrane. $\mathrm{GABA}_{\mathrm{A}} \mathrm{R}$ subunits are encoded by 19 different genes that have been grouped into eight subclasses based on sequence homology: Six $\alpha$, three $\beta$, three $\gamma$, one $\delta, \varepsilon$, $\theta, \pi$ and three $\varrho$ subunits (Luscher et al., 2011). The large number of $\mathrm{GABA}_{\mathrm{A}} \mathrm{R}$ subunits gives them an immense structural and physiological diversity. The subunit $\gamma 2$ is required for the clustering of $\mathrm{GABA}_{\mathrm{A}} \mathrm{Rs}$ at the postsynaptic membrane (Essrich et al., 1998). Glycinergic receptors are composed of five subunits, i.e. four $\alpha$ and one $\beta$ subunit. The heteromeric $\alpha 1 \beta$ glycine receptors are the most widely distributed in the brain and the spinal cord (Lynch, 2008). Glycine receptors act in the same manner as $\mathrm{GABA}_{\mathrm{A}} \mathrm{Rs}$ by creating a postsynaptic IPSP. At inhibitory synapses, there are no electron dense regions at the postsynaptic membrane, although certain proteins have been shown to be specifically present there. One of these proteins, gephyrin, clusters glycine receptors at the postsynaptic membrane (Meyer et al., 1995). Collybistin, a brain specific intracellular GDP/GTP-exchange factor, is able to attach gephyrin to the membrane by linking gephyrin and membrane lipids (Kins et al., 2000; Harvey et al., 2004; Kalscheuer et al., 2009). Gephyrin colocalizes specifically with $\mathrm{GABA}_{\mathrm{A}} \mathrm{R}$ in different brain regions and moreover, deletion of gephyrin results in a reduction of $\mathrm{GABA}_{\mathrm{A}} \mathrm{R}$ clusters in cultured neurons and brain sections (Kneussel et al., 1999).

\subsubsection{Synaptogenesis and synaptic maturation}

The formation of synapses, termed synaptogenesis, is the final step in the early development of the central nervous system. Synaptogenesis consists of several different steps. The axon growth cone seeks its target; a process controlled by chemoattraction or chemorepulsion stimuli such as Ephrins or Netrin. The next step is the differentiation of preand postsynaptic membrane specializations following the initial contact between the growth cone and its target. This synapse maturation consists of an upregulation of proteins targeted to the presynaptic side to assemble the presynaptic release machinery, as well as clustering of postsynaptic receptors by synaptic cell adhesion proteins. These cell adhesion proteins, which are present on both sides of the synapse, are required for synapse assembly and maintenance of synaptic cleft integrity (Gerrow and El-Husseini, 2006). 


\subsection{Neural oscillations}

\subsubsection{Role of network oscillations}

Neurons in the brain belong to a network and receive information from many different sources, all of which influence the response that each cell then produces. Neurons receive information through the different synapses present at their dendrites, cell body and axon. Depending on the type and the localization of these synapses, they can differentially influence the responses that the neurons are producing. Moreover, neurons can induce feedback connections that result in a synchronization of the neurons firing. A synchronous activity of a large population of neurons can lead to neural oscillations that can be observed by electroencephalography.

Consciousness, attention, perception and memory are thought to be dependent on this oscillatory network activity. Neurons are able to synchronize while firing and induce a rhythm. Oscillations can be detected in several brain regions such as the cortex, hippocampus, olfactory bulb and thalamus. In some cases, an ultra-synchronization of the neurons can lead to pathological disorders such as epilepsy. The neural oscillations are divided into frequency bands from the slow delta range $(0,5-3 \mathrm{~Hz})$ and theta range $(3-8 \mathrm{~Hz})$, to medium range alpha $(8-12 \mathrm{~Hz})$ and beta $(13-30 \mathrm{~Hz})$ oscillations, fast gamma oscillations $(30-80 \mathrm{~Hz})$ and ultrafast ripple oscillations $(80-200 \mathrm{~Hz})$. Each frequency range has been correlated with different brain states, and a band can characterize more than one state of consciousness. For example, the theta range is characteristic for an exploratory behavior as well as for rapid eye movement (REM) sleep.

\subsubsection{Gamma range oscillations}

The gamma oscillations $(30-80 \mathrm{~Hz})$ are closely correlated with higher brain functions. They are proposed to be involved in sensory processing and memory formation (Lisman and Idiart, 1995). Gamma oscillations can be detected in all cortical areas and in the hippocampus, where their amplitude is higher than in any other brain region. In the hippocampus, gamma oscillations have been correlated with specific behavior conditions such as exploratory behavior (Bragin et al., 1995), and performance during working memory tasks is modulated by the gamma oscillations (Fuchs et al., 2007). 


\subsubsection{Induction of gamma oscillations in the hippocampus}

Gamma oscillations can be induced by several methods in the hippocampus in vitro. These include electrical stimulation (Whittington et al., 1995) and chemical stimulation with agonists of metabotropic glutamate receptors (mGluRs) (such as ((s)-3,5dihydroxyphenylglycine (DHPG)), muscarinic acetylcholine receptors (mAChRs) (such as carbachol) and kainate receptors (such as kainate) (Whittington et al., 1995; Fisahn et al., 1998; Gillies et al., 2002; Fisahn et al., 2004). Multiple mechanistic pathways appear to be involved in the generation of gamma oscillations in vitro. Oscillations induced by activation of kainate receptors and mGluRs are dependent principally on GABAergic inhibition mediated by $\mathrm{GABA}_{\mathrm{A}}$ receptors, since they can be blocked by application of bicuculline but cannot be inhibited by AMPA receptor antagonists (Whittington et al., 1995; Fisahn et al., 2004). In contrast, the oscillations induced by Carbachol can be blocked by both AMPA receptor and $\mathrm{GABA}_{\mathrm{A}}$ receptor antagonists, implying that they are dependent on both excitatory and inhibitory pathways (Fisahn et al., 1998). This difference may be explained by the receptor distribution in the network, since mGluRs and kainate receptors are present on interneurons, whereas the mAChRs are localized on hippocampal pyramidal cells (van Hooft et al., 2000; Fisahn et al., 2002; 2004).

\subsubsection{Cells involved in gamma oscillations}

The hippocampus is divided between the dentate gyrus and the Ammon's horn field (Cornu Ammonis), which is subdivided into four parts the Cornu Ammonis 1-4 (CA1-4). The hippocampal circuit consists of a tri-synaptic neuronal circuit. In the first part of this circuit, axons arrive from the entorhinal cortex and form synapses onto the granule cells of the dentate gyrus (perforant path). The granule cell axons project to area CA3 and form synapses onto the dendrites of CA3 pyramidal cells (mossy fiber path). The axons from these pyramidal cells in turn project to and synapse onto the dendrites of the CA1 pyramidal cells (Schaeffer collateral path). Finally, these cells send axons into the subiculum or the entorhinal cortex, where they exit the hippocampal circuitry. The CA3 and CA1 network architecture is similar to that of the cortex, with excitatory principal neurons that process, store and retrieve 
the information and local inhibitory interneurons that modulate this processing (Treves and Rolls, 1994).

Inhibitory interneurons are classified according to the soma shape and localization and to specific protein expression such as parvalbumin (PV) or calbindin. The generation of gamma oscillations depends on the inhibitory function of certain subsets of these GABAergic interneurons (Fisahn et al., 2004), including the fast spiking PV positive basket cells. These cells are abundant in the hippocampus (Freund and Buzsáki, 1996), present in the pyramidal cell layer of CA1 and CA3, and connected to the perisomatic region of the pyramidal cells (Cobb et al., 1995; Miles et al., 1996). The fast spiking phenotype of the PV basket cells allows them to fire at similar frequencies as their excitatory input (Jonas et al., 2004). Other PV positive interneurons with a different shape can fire in the gamma range, such as the axoaxonic cells and the bistratified cells (Hájos et al., 2004; Gloveli et al., 2005; Gulyás et al., 2010). Other GABAergic interneurons such as the oriens/lacunosum-moleculare cells fire in a slower range (Hájos et al., 2004; Gloveli et al., 2005). Further GABAergic cell types might be involved in the propagation of oscillations in the hippocampus, such as the trilaminar cell (Gloveli et al., 2005), and cholesystokinin positive cells can modulate oscillations (Freund and Katona, 2007; Tukker et al., 2007).

\subsubsection{Hippocampal circuitry involved in gamma oscillations}

The CA3 region is a useful model system to study gamma oscillations in vitro due to the amplitude of the oscillations, which is significantly higher than in CA1. CA3 pyramidal cells receive afferents from the dentate gyrus, the entorhinal cortex and from other CA3 pyramidal cells, and they are also closely connected to and modulated by local inhibitory circuits. Generation of oscillations is dependent on the synaptic contact and the axonal collaterals between the pyramidal cells and the inhibitory interneurons that allow feedforward and feedback inhibition mechanisms, which are required for the generation of the oscillation (Mann et al., 2005b; Hájos and Paulsen, 2009). The application of kainate depolarizes inhibitory interneurons and causes them to fire in a range that synchronizes the pyramidal cells (Fisahn et al., 2004). The activated pyramidal cells, in turn, excite the interneurons (Glickfeld and Scanziani, 2006). The excitatory input from the pyramidal cells is located on the dendritic spines, whereas the inhibitory inputs can be present on the soma, axon or the 
dendritic shaft. The different localization of inhibitory synapses reflects the variety of inhibitory interneurons in the hippocampus: PV positive baskets cells preferentially form synapses onto the soma of the pyramidal cells, whereas somatostatin positive interneurons preferentially synapse onto the axon initial segment of these cells for a modularity effect on the neuron response to the stimulus (Freund, 2003; Mann and Paulsen, 2007).

\subsection{Neuroligin and synapse function}

\subsubsection{Synaptic cell adhesion proteins}

The synaptic communication required to induce neural oscillations depends on close interaction between the excitatory pyramidal cells and the inhibitory interneurons. Synapse integrity, in turn, is necessary for efficient neurotransmission and depends on the proper functioning of processes like synaptogenesis and synapse maturation. Cell adhesion proteins have been identified to play key roles in these processes. Synaptic cell adhesion proteins are pre- and postsynaptic transmembrane proteins that bridge the synaptic cleft and interact with each other to form direct contacts between pre- and postsynaptic neurons. These molecules are believed to be essential for the establishment, maturation and plasticity of synapses, and they are able to activate and recruit synaptic proteins required for synapse function (Missler et al., 2012). Several adhesion proteins have been identified at synapses, including the neurexin (NX) neuroligin (NL) complex (Ichtchenko et al., 1995; 1996), Ig-domain proteins such as the SynCAMs, leucine-rich repeat proteins (LRRTMs), and receptor tyrosine kinases and phosphatases. The importance of studying NX-NL complexes became particularly clear when mutations in genes coding for these proteins were found to be associated with several neuropsychiatric disorders such as autism (Jamain et al., 2003; Sudhof, 2008).

\subsubsection{Function of the neuroligin-neurexin complex}

NL and NX are type I transmembrane proteins, composed of an N-terminal extracellular domain and a transmembrane domain followed by a C-terminal intracellular domain. Three NX genes are present in the mammalian genome, with two promoters that induce the synthesis of a long $\alpha$-isoform and a short $\beta$-isoform (Tabuchi and Sudhof, 2002). NXs can be spliced into over a thousand isoforms (Boucard et al., 2005). The extracellular 
domain of $\alpha-\mathrm{NX}$ is composed of six laminin, NX, sex-hormone-binding protein (LNS) domains (Rudenko et al., 1999) and three epidermal growth factor (EGF)-like domains, while $\beta$-NX only contains the sixth LNS domain without EGF-like sequences (Missler and Sudhof, 1998). NXs are considered to be presynaptic but one study emphasized a potential role at the postsynaptic side (Taniguchi et al., 2007). The intracellular domain of NX contains a PDZbinding domain that can interact with presynaptic scaffolding proteins (Craig and Kang, 2007; Sudhof, 2008; Krueger et al., 2012). The intracellular complex formed by NX induces the stabilization of nascent presynaptic terminals (Sankaranarayanan et al., 2003). Indeed, $\beta$-NX binds to calcium/calmodulin-dependent serine protein kinase (CASK), which forms a macromolecular complex with voltage-gated $\mathrm{Ca}^{2+}$ channels (Butz et al., 1998; Maximov et al., 1999).

NLs were initially discovered as NX ligands (Ichtchenko et al., 1995) and are composed of an extracellular N-terminal acetylcholinesterase-like domain, a glycosylated linker region that connects to the transmembrane domain and a short $\mathrm{C}$-terminal region with a PDZ-binding domain. The acetylcholinesterase-like domain is inactive in NL because of the lack of the complete catalytic triad in the active site (Comoletti et al., 2003; Dean et al., 2003). NL1-3 are alternatively spliced at a single conserved position (site A), and NL1 can be spliced at a second site (site B) (Ichtchenko et al., 1996; Boucard et al., 2005). NLs form dimers through their extracellular domain (Comoletti et al., 2003), and this dimerization is required for the association with neurexins (Dean et al., 2003).

NL and NX interact with each other through their extracellular domain. This interaction takes place between the acetylcholinesterase-like domain of NL and the membrane-proximal LNS domain of NX (the sixth LNS domain in $\alpha-\mathrm{NX}$ and the only one in $\beta$-NX) (Krueger et al., 2012). The details of the interaction between NX and NL have been elucidated by the analysis of crystal structures of NX-NL complexes. NLs create a central dimer and two NX monomers bind to two identical surfaces of the NL dimer to form a heterotetramer (Arac et al., 2007; Comoletti et al., 2007; Fabrichny et al., 2007). The binding between $\mathrm{NX}$ and $\mathrm{NL}$ is $\mathrm{Ca}^{2+}$-mediated and can be modulated by $\mathrm{pH}$ or ionic strength (Chen et al., 2007; Reissner et al., 2008). 
In rodents, there are four NLs isoforms, NL1-4 (Ichtchenko et al., 1995; 1996; Jamain et al., 2008) whereas in humans, there are five, NL1-3, NL4X and NL4Y (Jamain et al., 2003). Localization of NL1-3 mRNA by in situ hybridization demonstrated a ubiquitous expression throughout the brain (Varoqueaux et al., 2006). This mRNA expression, like the expression of NL4 protein, is detectable from the early postnatal stages until adult stages (Varoqueaux et al., 2006; Jamain et al., 2008). Interestingly, the NL isoforms in rodents are localized to different subtypes of synapses. NL1 is localized specifically to glutamatergic synapses (Song et al., 1999), whereas NL2 is present at inhibitory synapses (Varoqueaux et al., 2004). The distribution of NL3 is still unclear. It has been show that this isoform can be present at excitatory and inhibitory synapses in cultured neurons (Budreck and Scheiffele, 2007; Levinson et al., 2010), but no in vivo data have been published to support this. The distribution and the function of NL4 have not yet been studied at central nervous system synapses.

To understand the function of the NL-NX complex, several studies involving overexpression of NLs in non-neuronal cells showed that NLs induce the formation of presynapses in contacting co-cultured neurons (Scheiffele et al., 2000; Graf et al., 2004). In cultured neurons, overexpression of NXs and NLs induces an increase in synapse number (Prange et al., 2004; Chubykin et al., 2007). However, the analysis of triple knockout mice lacking all three $\alpha$-NXs (Missler et al., 2003) or NL1-3 (Varoqueaux et al., 2006) did not reveal any dramatic changes in synapse numbers in the brain in vivo. Rather, genetic deletion of NL1-3 impaired synapse function by reducing GABAergic/glycinergic and glutamatergic synaptic transmission in the brainstem (Varoqueaux et al., 2006). These findings indicated that the NX-NL complex is not essential for synapse formation but crucial for synapse maturation. Accordingly, single deletion of NL1 impairs spontaneous excitatory postsynaptic currents (EPSCs) (NMDA and AMPA receptor) (Chubykin et al., 2007; Mondin et al., 2011), (Shipman et al., 2011) and the lack of NL2 resulted in a reduction of IPSCs (Chubykin et al., 2007; Fu and Vicini, 2009; Poulopoulos et al., 2009; Jedlicka et al., 2011); (Gibson et al., 2009). On the other hand, overexpression of NL1 or NL2 in vitro induces an increase of NMDAR EPSCs (Chubykin et al., 2007; Ko et al., 2009) and IPSCs, respectively (Hines et al., 2008; Fu and Vicini, 2009). These data indicate that NLs are important for synapse maturation, plasticity and maintenance (Krueger et al., 2012). 
All NL isoforms contain a C-terminal intracellular domain that consists of a conserved cytoplasmic PDZ-binding motif that is able to recruit postsynaptic scaffold proteins such as PSD-95, synaptic scaffolding molecule (S-SCAM) and thus, indirectly, glutamate receptors (Figure 1.1) (Baudouin and Scheiffele, 2010; Krueger et al., 2012). At inhibitory synapses, NLs can recruit the inhibitory scaffolding protein gephyrin and collybistin, and, through the activation of this complex, $\mathrm{GABA}_{\mathrm{A}} \mathrm{R}$ and GlyR (Figure 1.1) (Meyer et al., 1995; Graf et al., 2004; Poulopoulos et al., 2009). The NX-NL complex also plays a role in synapse stabilization, physically stabilizing the nascent synapse (Chen et al., 2010; Arstikaitis et al., 2011). The early interaction between $\beta$-NX and NL1 can recruit further NL1 molecules that diffuse in the plasma membrane (Barrow et al., 2009), which in turn stabilizes dendritic filopodia (Chen et al., 2010; Arstikaitis et al., 2011). The scaffolding protein PSD-95 is then recruited to sites of contact between $\beta$-NX and NL1 by a conserved cytoplasmic PDZ-binding motif present in all NLs (Figure 1.1) (Irie et al., 1997; Hirao et al., 1998; Meyer et al., 2004; Heine et al., 2008; Barrow et al., 2009; Mondin et al., 2011). NL1 and PSD-95 are mutually dependent with regard to their localization, since the knockdown of PSD-95 decreases NL1 at the synapse (Levinson et al., 2010). The binding of NL1 to PSD-95 induces the recruitment of functional AMPARs by trapping them from the membrane diffusible AMPAR pool. The clustering of receptors at the postsynapse probably involves auxiliary proteins associated with AMPARs, such as stargazin (Figure 1.1) (Heine et al., 2008; Mondin et al., 2011). Interestingly, NMDARs are recruited to the synapse by a mechanism that does not involve PSD-95 but instead depends on NMDARs pre-clustered with NL1. This mechanism involves PDZ-domain scaffolding proteins such as S-SCAM (Figure 1.1) (Barrow et al., 2009). In certain experimental settings, the $\beta$-NX-NL1 complex is sufficient to recruit AMPARs and NMDARs to the nascent synapses and to contribute to their maturation. 
a

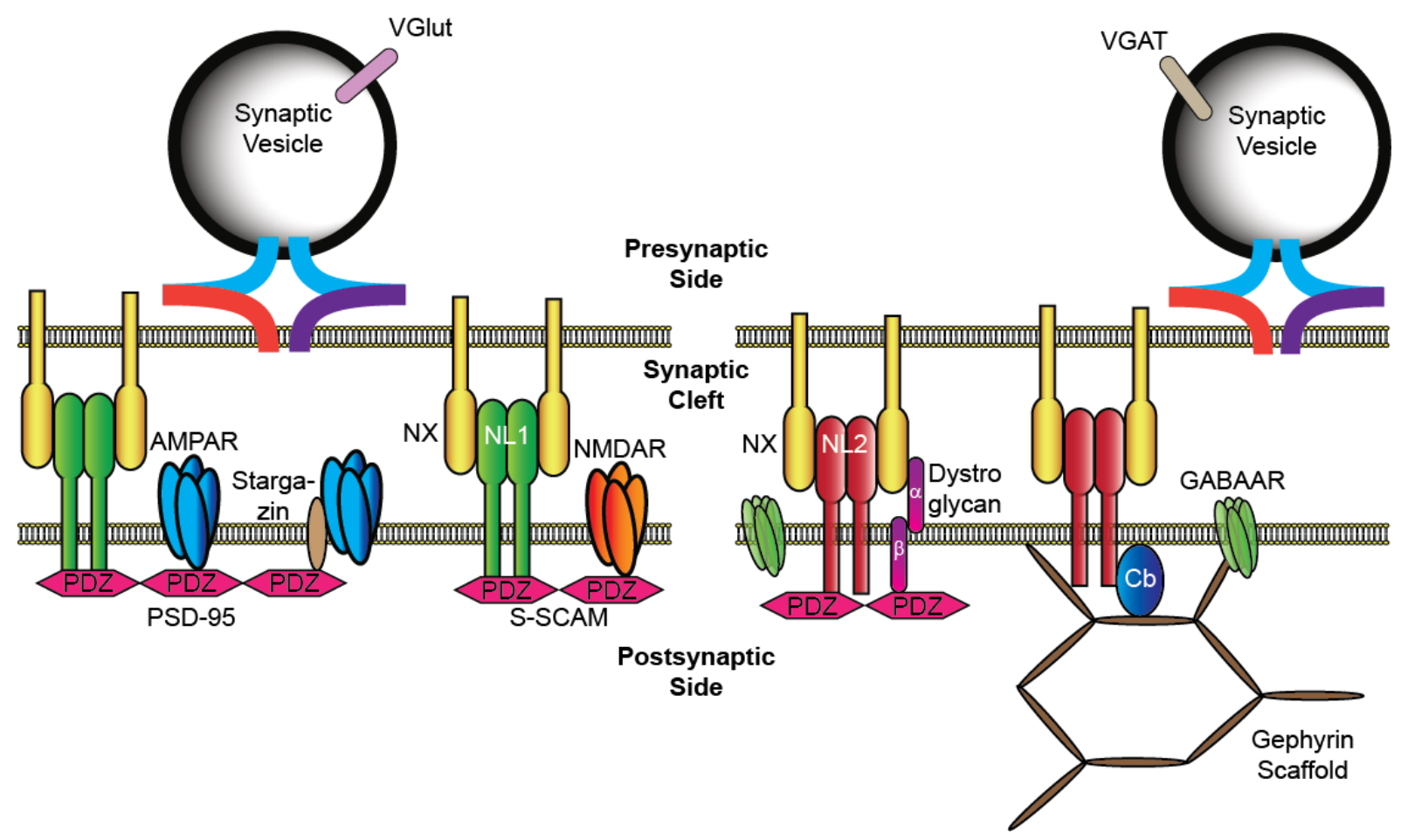

Figure 1.1: Synaptic organization of neurexin neuroligin interactions at (a) excitatory synapses and (b) inhibitory synapses (adapted from Krueger et al., 2012). The neurexin neuroligin bridging is shown in yellow (neurexin) and red or green (neuroligin). The scaffolding proteins that interact with neuroligin are shown in purple, pink, blue and brown. Abbreviations: AMPAR, AMPA receptor; $\mathrm{Cb}$, collybistin; GAGAAR, GABA $\mathrm{A}_{\mathrm{A}}$ receptor; NL, neuroligin; NMDAR, NMDA receptor; NX, neurexin; PDZ, PSD-95/disclarge/zonaoccludens-1 domain; PSD-95, postsynaptic density protein of $95 \mathrm{kDa}$; S-SCAM, synaptic scaffolding molecule; VIAAT, vesicular GABA/glycine transporter; VGluT, vesicular glutamate transporter.

At inhibitory synapses, the interaction between NXs and NL2 induces clustering of NL2 (Papadopoulos et al., 2008), which then leads to recruitment of the inhibitory scaffolding protein gephyrin (Figure 1.1) (Graf et al., 2004). NL2 is further capable of interacting with an intracellular membrane protein called collybistin, which binds and accumulates gephyrin at the synapses (Papadopoulos et al., 2008; Poulopoulos et al., 2009). The complex formed by NL2-gephryin-collybistin can recruit $\mathrm{GABA}_{\mathrm{A}} \mathrm{Rs}$ and GlyRs to the inhibitory postsynaptic membrane for synaptic maturation (Figure 1.1). NL2 can also cluster $G_{A B} A_{A} R$ at synapses in the absence of gephyrin or collybistin, but the underlying mechanism is still unknown (Dong et al., 2007; Fu and Vicini, 2009; Hoon et al., 2009). The NL2-gephyrin-collybistin complex was observed at inhibitory perisomatic synapses (Figure 1.2) (Poulopoulos et al., 2009) and on inhibitory axo-dendritic synapses. NL2 can bind the scaffolding protein S-SCAM, which in turn can recruit $\beta$-dystroglycan (Figure 1.2) (Sumita et al., 2007). Indeed, GABA $A_{A}$ 
subtypes differ between perisomatic and dendritic synapses, and $\beta$-dystroglycan could play a key role in the $\mathrm{GABA}_{\mathrm{A}} \mathrm{R}$ clustering at different synapses (Panzanelli et al., 2011). NL2 is not present at all inhibitory postsynapses, and for some synapses, such as the inhibitory distal axo-dendritic synapses in pyramidal cells, loss of NL2 does not affect their function (Gibson et al., 2009). It is possible that other NL isoforms or some other adhesion molecules are involved in the formation and function of these synapses.

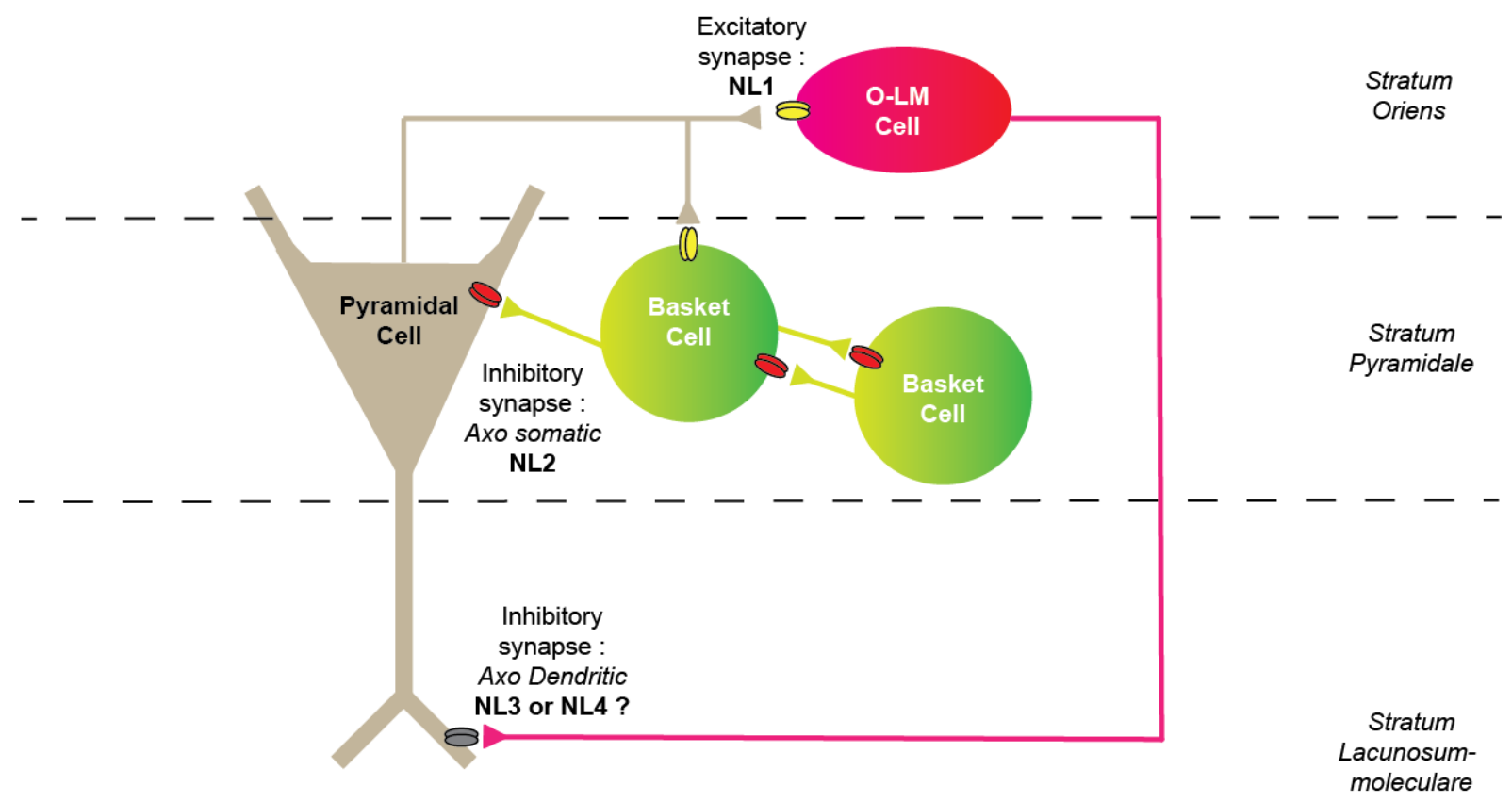

Figure 1.2: Schematic diagram of the anatomical connectivity with the neuroligins (NLs) isoforms present at each synapse between pyramidal cells (grey) and selected interneuron subtypes (pink and green) within the CA3 network.

Concerning NL3, the density of synapses and their physiological function have been studied using NL3 knockout mice, and no changes were observed in synapse number or synaptic transmission in the hippocampus and the barrel cortex (Tabuchi et al., 2007).

These studies cumulatively indicate that although NX-NL complexes are sufficient to trigger synapses formation in vitro, they are not essential for synapse formation in vivo, where they may be more relevant for the maturation and maintenance of synapses (Ichtchenko et al., 1996; Irie et al., 1997; GARNER et al., 2002). 


\subsubsection{Role of neuroligins at mature synapses}

In addition to its role in synapse development, the NX-NL complex is believed to be functionally important for mature synapses. In the amygdala, during normal development and plasticity, NMDAR-mediated transmission is dependent on NL1 (Kim et al., 2008; Jung et al., 2010). Further, the deletion of NL1 impairs LTP in the CA1 region of the hippocampus (Blundell et al., 2010; Dahlhaus et al., 2010). The deficit of NL1 in the amygdala of rat is accompanied by behavioral deficits in learning and memory (Kim et al., 2008), and a deficit was observed in a memory task in the NL1 knockout mice (Blundell et al., 2010).

\subsubsection{Role of neuroligins in neurodevelopmental disorders}

Mutations in the NL3 and NL4 genes have been identified in patients with ASD, Asperger syndrome and mental retardation (Jamain et al., 2003; Laumonnier et al., 2004; Yan et al., 2005). In one family, a single amino acid substitution was observed in NL3, which modifies an arginine residue into a cysteine (R451C) in the extracellular domain of the protein (Jamain et al., 2003). This mutation has been shown to induce the partial retention of the protein at the endoplasmic reticulum (Jamain et al., 2003; Comoletti et al., 2004).

While to date only one mutation in NL3 has been directly associated with autism, at least ten separate mutations in the NL4 gene were linked to ASDs (Jamain et al., 2003; Laumonnier et al., 2004; Yan et al., 2005; Zhang et al., 2009). One of these mutations is a frameshift mutation (1186insT) that induces a premature termination of the protein (Jamain et al., 2003). Several other studies highlighted missense mutations in the acetylcholinesterase and cytoplasmic domains (Yan et al., 2005). Another study found that a two base pair deletion of the NL4 gene was discovered in ASD patient, and this mutation led to a truncation of the C-terminal transmembrane domain (Laumonnier et al., 2004). A further study based on a NL4 human mutation $(\mathrm{R} 87 \mathrm{~W})$ associated with classical ASD showed that this mutation induced the retention of the NL4 protein in the endoplasmic reticulum in non-neuronal cells and neurons, and this abolished the functionality of NL4 in synapse formation and function (Zhang et al., 2009). Finally, one NL4 mutation was found in a case of mental retardation without any correlation with ASD (Laumonnier et al., 2004). 
Overall, mutations in NL3 and NL4 explain less than $0.1 \%$ of all ASD diagnoses (Jamain et al., 2003; Laumonnier et al., 2004; Vincent et al., 2004; Gauthier et al., 2005; Yan et al., 2005; Buxbaum, 2009). Interestingly, however additional genes encoding synaptic proteins seem to be mutated in ASD (Persico and Bourgeron, 2006; Sudhof, 2008; Bourgeron, 2009), including the genes encoding the postsynaptic scaffolding proteins SHANK2 and SHANK3 (Durand et al., 2007; Berkel et al., 2010), as well as members of the presynaptic neurexin family (Kim et al., 2008; Gauthier et al., 2011). These findings imply that abnormalities in synapse development and function may be a common mechanism in ASDs, and that the study of synaptic proteins, and particularly of NL4, is likely to provide useful insights into the general principles underlying autism.

\subsubsection{Role of neuroligins in ASD using animals models}

Further insights into the role of neuroligins in ASD can be obtained by using NL mutant mouse models to assess the effect of alterations in NL expression on morphological, physiological and behavioral phenotypes related to autism.

One such model is the NL3 knockout mouse. These mice showed a deregulation of ultrasound vocalization, as well as a lack of social novelty preference that is characteristic of some forms of ASDs (Radyushkin et al., 2009). Moreover, A NL3 Arg451Cys (R451C) knock-in mouse was generated to mimic the NL3 mutation described in individuals with an ASD (Tabuchi et al., 2009). Interestingly, this mutation was found to be a NL3 gain of function mutation in mouse (Tabuchi et al., 2009). These mice showed an impairment in social interaction (Tabuchi et al., 2009), an increased synaptic inhibition in the somatosensory cortex and strikingly, as well as an enhancement of excitatory synaptic transmission and a change in the glutamate receptor composition of synapses in the hippocampus, together with an increase in the branching in the stratum radiatum (str. rad) of the hippocampus CA1 region (Tabuchi et al., 2007; Etherton et al., 2011). These findings provided the first evidence that a NL mutation can act by different mechanisms at different synapses, depending on the synaptic context. 
Like the NL3 mutant mice, NL4-KO mice provide a valuable model for studying the role of NL4 in autism-related phenotypes. Indeed, NL4-KO mice show selective perturbations in social interaction and ultrasonic vocalizations, which are thought to mimic the impairments in social interaction and social communication observed in ASDs (Jamain et al., 2008). By Western blot, it was observed that the NL4 protein is expressed everywhere throughout the rodent central nervous system, and that the total volume of the brain, especially of the brainstem and the cerebellum, is slightly decreased in the NL4-KO mice (Jamain et al., 2008). The expression level of NL4 protein in the central nervous system is substantially lower than that of the three others isoforms, making up only $3 \%$ of the total NLs in adult mice (Varoqueaux et al., 2006). However, prior to this thesis work, the detailed localization of the NL4 protein in the central nervous system, as well as the consequences of NL4 deletion on synapse density and physiology, was unknown.

The NL4 gene is conserved in most vertebrates, but the mouse NL4 gene is highly divergent from other species, although it maintains the same properties, such as its ability to bind NX (Bolliger et al., 2008). This phenomenon may be explained by the fact that NL4 is less stringently controlled by evolution, since the NL4-KO has rather mild consequences, even in humans where its loss causes comparatively moderate neurodevelopmental deficits (Bolliger et al., 2008).

In summary, the function of NL4 in the brain is still rather enigmatic. Indeed, no in vivo studies have been performed so far to determine the localization and synaptic distribution of NL4 in the brain, and even less is known concerning the effects of the deletion of NL4 on synapse, neuron and brain function.

\subsection{Aim of the present study}

The primary aim of the present study was to explore the specific expression and distribution of NL4 in the central nervous system, and to examine the role of this protein in the establishment, function and maintenance of neural networks.

Specifically, the localization of NL4 in the rodent brain was studied using immunostaining methods and the functional consequences of NL4 deletion in mice were 
examined, focusing on those brain regions that are relevant for the study of autism. In different brain areas, the effects of NL4 loss on synapse density and the levels of others synaptic proteins were investigated. Moreover, the involvement of NL4 in modulating neural network function was studied in the hippocampus.

Taken together, this study is the first to investigate the localization and the role of NL4 in a morphologically intact model system. 


\section{Materials and Methods}

\subsection{Animals}

For this study, neuroligin 4 knockout (NL4-KO) mice and wild type (WT) littermate controls (Jamain et al., 2008) were used and bred on a C57BL/6 background in the animal facility of the Max Planck Institute for Experimental Medicine. Mice used in experiments were age and sex matched. Tail biopsies were taken from each animal used in experiments to ensure correct allocation of genotype. This investigation was carried out in agreement with the guidelines for the welfare of experimental animals issued by the Federal Government of Germany and the Max Planck Society.

\subsection{Immunohistochemistry}

\subsubsection{Tissue Preparation}

Immunohistochemistry was carried out on 8 to 12 week old WT and NL4-KO mice. Animals were deeply anesthetized with Isofluorane (DeltaSelect) and then rapidly decapitated. The whole brain was removed carefully from the skull. For spinal cord dissection, hydraulic expulsion was used (Chéry and de Koninck, 1999). Briefly, the spinal cord was expelled out of the lumbar region using a syringe containing phosphate buffered saline (PBS). Sections of the extracted spinal cord were taken from the thoracic part to the sacral part.

Spinal cord and brain were then quickly frozen in a $-35^{\circ} \mathrm{C}$ isopentane bath (Carl-Roth). Brains were subsequently mounted on a specimen disc and embedded in Tissue-Tek ${ }^{\circledR}$ using a cryostat (Leica), and sagittal or coronal brains sections $(14 \mu \mathrm{m})$ were cut. The sections were directly mounted on glass slides and dried for $30 \mathrm{~min}$ at room temperature.

Fixation was performed using two different methods. In order to label postsynaptic proteins, slides were submerged in a $-20^{\circ} \mathrm{C}$ methanol bath for 5 min (Schneider Gasser et al., 2006). In order to co-label post- and presynaptic proteins, the sections were immersed in $4 \%$ 
paraformaldehyde (PFA, SERVA Electrophoresis) in $0.1 \mathrm{M}$ PB for $10 \mathrm{~min}$ and then heated to $95^{\circ} \mathrm{C}$ for $30 \mathrm{~min}$ in a sodium citrate buffer (10 mM Sodium Citrate, $0.05 \%$ Tween 20, $\mathrm{pH} 8$ ) (Papadopoulos et al., 2008). Each antibody was tested with both methods and thereafter used with the method yielding the better specific staining.

\subsubsection{Immunostaining}

Following fixation and subsequent washing steps, the sections were blocked with 5\% goat serum and 0.1\% Triton X-100 (Roche Applied Science) for $1 \mathrm{~h}$ at room temperature. This blocking solution was also used for the primary and secondary antibody incubations. The dilutions that were used for the primary and the secondary antibodies are indicated in Table 2.1 and Table 2.2. Incubation of the primary antibodies was carried out at $4^{\circ} \mathrm{C}$ overnight, followed by incubation with secondary antibodies for $2 \mathrm{~h}$ at room temperature, with $3 \times 5 \mathrm{~min}$ washes using PBS between every step. Lastly, sections were mounted using a water basedmounting medium (Polysciences Inc.).

\subsubsection{Image capture and analysis}

Global NL4 localization in the brain was characterized by capturing lowmagnification brain overview images with a binocular (Leica MZ16) (Figure 3.1, 3.2, 3.3). High magnification single-plane confocal images were recorded using a TCS-SP2 or TCSSP5 inverted confocal microscope (Leica Microsystems) and taken with a 63X objective (N.A 1.4). A digital zoom factor of 1 was used for protein localization within a brain region area, while a zoom factor of 8 was used for the high magnification images that were necessary for synaptic quantification or analysis of colocalization with other synaptic markers.

The figures presented in this work were processed using Photoshop CS5 (Adobe). The only modification from the original data was to readjust the brightness, contrast and tonal range of the images. The same settings were always applied to matched pairs of WT and NL4-KO mice.

For quantification and colocalization experiments, images were processed using Image J (http://rsbweb.nih.gov/ij/) (Figure 2.1). For each picture, a threshold was first defined to generate a binary image. This threshold was defined by determining two points from a 
histogram illustrating the distribution of the pixel intensity. The first point, B, was the background pixel intensity (defined as the intensity with the largest number of pixels). The second point, M, was the maximum pixel intensity. The following mathematical formula was then applied to calculate the individual image threshold:

Threshold $=\mathrm{B}+((\mathrm{M}-\mathrm{B}) \mathrm{x}$ Factor $)$

The factor present in the formula was defined based on empirical initial observations, but always remained the same across experiments for a given antibody labeling.

Once the binarized images had been obtained, an algorithm called Watershed Segmentation was applied, which automatically separates particles that are touching after application of the threshold. Finally, the command 'Analyze Particles' was used to count the number of particles per image that were considered to be synapses. A synapse was defined to be a particle that consisted of 30 or more adjoining pixels $(1.75 \mu \mathrm{m})$.

For colocalization experiments, the threshold was evaluated separately for each channel (488 or $555 \mathrm{~nm}$ ) according to the method describe above. An Image J plugin called Colocalization was used. This plugin made it possible to manually set the threshold for every channel. Pixels were considered to be colocalized if the intensity value in both channels corresponded to the value of the threshold or higher. Colocalized pixels were indicated in white on the image, with the maximum intensity value for an 8 bit image (255). The number of colocalized particles consisting of at least 15 adjoining pixels was counted.

Since the number of synaptic puncta in many images was very high, a certain number of puncta were likely to colocalize by random coincidence. To control for this effect, one of the images (488 or $555 \mathrm{~nm}$ channel) was rotated by $90^{\circ}$, and the number of randomly colocalized particles was determined as above. To obtain the final number of colocalized particles, the number of colocalized particles due to random coincidence after rotation was subtracted from our obtained calculated value to assess the true colocalization. 
A. Determination of the threshold

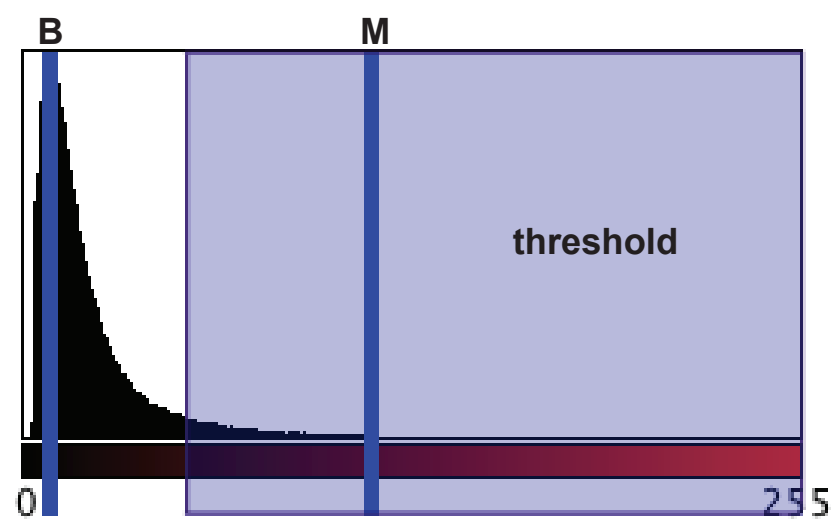

Count: $262144 \quad$ Min: 1

Mean: 22.212
Threshold $=B+((M-B) X$ factor $)$

B. Number of cluster per picture

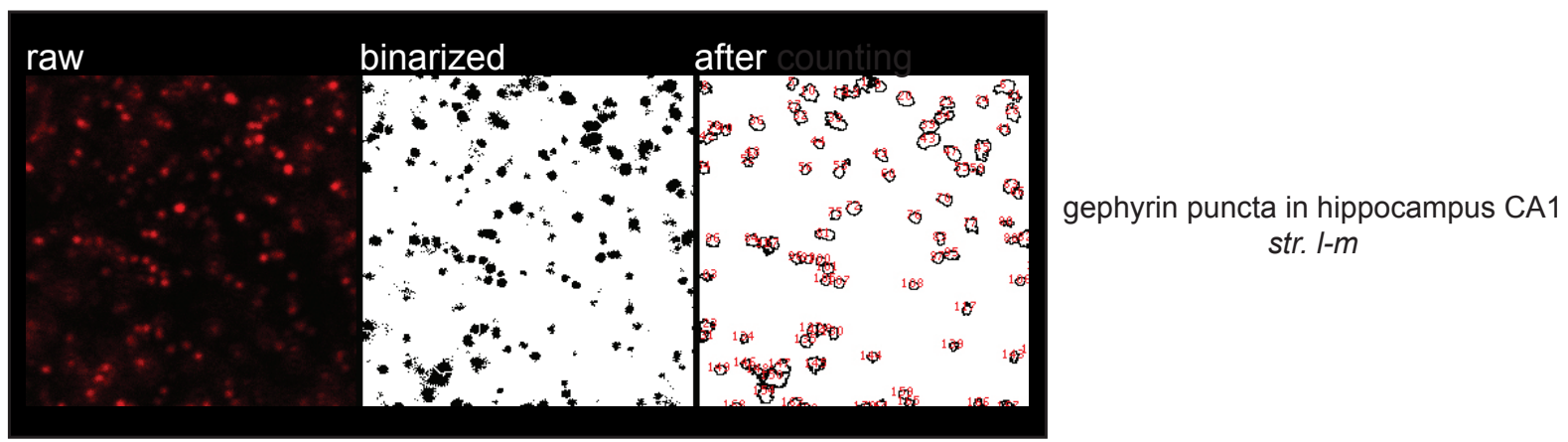

C. Colocalization counting

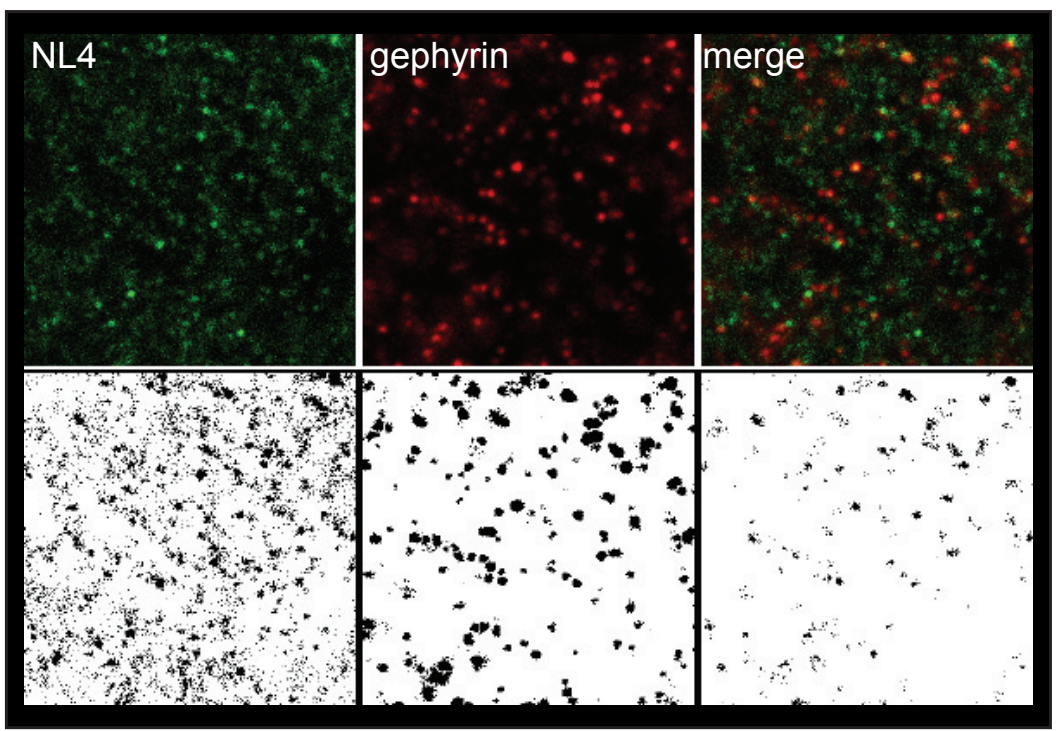

Figure 2.1: Quantification of puncta numbers and percentage of colocalization between two synaptic markers. (A) Histogram of the pixel intensity distribution used to set a threshold (left) with the mathematical formula used to calculate this threshold (right). (B) Illustration of the method used for puncta quantification. Single channel images were binarized according to the threshold set above, and particle numbers were then evaluated. (C) illustration of the method used for colocalization of two channels. Both channels are binarized according to the threshold set previously. Subsequently, the two channels are merged, and a 
binarized image is generated from the merged image. This binarized image includes all pixels that are positive in both individual channels in the same location of the image.

\subsection{SDS PAGE and Western immunoblotting}

\subsubsection{Preparation of the homogenate}

Mice were sacrificed by rapid decapitation, brains were removed from the skull and placed in ice-cold high sucrose dissection buffer $(87 \mathrm{mM} \mathrm{NaCl}, 2.5 \mathrm{mM} \mathrm{KCl}, 1.25 \mathrm{mM}$ $\mathrm{KH}_{2} \mathrm{PO}_{4} ; 25 \mathrm{mM} \mathrm{NaCO} 3,0.5 \mathrm{mM} \mathrm{CaCl}_{2}, 7 \mathrm{mM} \mathrm{MgCl}$, $75 \mathrm{mM}$ Sucrose, $10 \mathrm{mM}$ Glucose, 1.3 $\mathrm{mM}$ ascorbic acid, supplemented with protease inhibitors, Calbiochem). The brains were then transferred into a mouse brain matrix (Braintree Scientific) and $1 \mathrm{~mm}$ coronal sections were sectioned and immediately frozen on dry ice. The brain sections were then thawed and specific regions of interest were rapidly isolated (somatosensory cortex, hippocampus, striatum and brainstem). The brain regions were then frozen in liquid nitrogen and stored at $80^{\circ} \mathrm{C}$ until they were homogenized in SDS lysis buffer (containing $1 \%$ protease inhibitors) using an all-glass dounce homogenizer (0.013-0.064 mm clearance, Kontes Glass Co.).

\subsubsection{SDS-PAGE and immunoblotting}

Total protein concentration of each brain sample was determined using a BCA assay (Pierce). A protein concentration corresponding to 5-10 $\mu \mathrm{g}$ per sample was loaded for SDSPAGE. Proteins were denatured by heating to $95{ }^{\circ} \mathrm{C}$ in Laemmli buffer (2\% SDS, $62.5 \mathrm{mM}$ Tris, $10 \%$ glycerol, $1 \% \beta$-mercaptoethanol, $0.01 \%$ bromophenol blue, $\mathrm{pH} \mathrm{6.8)} \mathrm{for} 5 \mathrm{~min}$. Samples were resolved on $10 \%$ SDS-PAGE gels, transferred onto nitrocellulose membranes by wet transfer and stained for total protein using a Memcode assay (Pierce). After evaluation of the total protein concentration per sample, nitrocellulose membranes were blocked in 50\% LiCor blocking buffer (LiCor Biosciences) in PBS for $1 \mathrm{~h}$ and were incubated with primary antibodies in primary antibody dilution buffer (50\% LiCor blocking buffer and $0.1 \%$ Tween20 in PBS) under agitation at $4^{\circ} \mathrm{C}$ overnight. After washing using $0.1 \%$ Tween-20 in PBS, membranes were incubated with secondary antibodies in secondary antibody dilution buffer (50\% LiCor blocking buffer, 0.1\% Tween-20 and 0.01\% SDS in PBS) under agitation for $1 \mathrm{~h}$ at room temperature. Blots were then washed as previously and scanned on an Odyssey Infrared Imager (LiCor Biosciences). Signal intensity for each sample was quantified using 
the Odyssey 2.0 software. Sample intensity value was normalized to the total protein loading value of that sample lane by comparison to the Memcode assay data. In addition, results were then normalized to the average blot value to correct for the variance between blots.

\subsection{Antibodies}

\subsubsection{Primary antibodies}

Table 2.1: Primary antibodies used for this project: 'Antigen' refers to the protein against which the antibody was raised. 'Type' refers to whether the antibody was polyclonal (i.e. containing a heterogeneous complex mixture of antibodies of different affinity) or monoclonal (i.e. a obtained from a single hybridoma cell line and therefore containing only antibodies with an identical epitope affinity). 'Host' refers to the species from which the antibody was taken via serum (for the polyclonal antibodies) or immune cells (for the monoclonal). The columns 'immunohistochemistry' (IHC) and 'western blots' describe the working dilution used for each type of experiment in this study. The last column gives the name of the company from which the antibodies were purchased.

\begin{tabular}{|c|c|c|c|c|c|}
\hline Antigen & Type & Host & IHC & $\begin{array}{l}\text { Western } \\
\text { Blot }\end{array}$ & Company \\
\hline NL4 & Polyclonal & Rabbit & $1 / 1000$ & $1 / 2000$ & \\
\hline Gephyrin & $\begin{array}{l}\text { Monoclonal } \\
\text { (3b11) }\end{array}$ & Mouse & $1 / 1000$ & & Synaptic Systems \\
\hline PSD-95 & $\begin{array}{l}\text { Monoclonal } \\
\text { (K28/43) }\end{array}$ & Mouse & $1 / 3000$ & & Neuromab \\
\hline GlyR & $\begin{array}{l}\text { Monoclonal } \\
(\mathrm{mAb} 4 \mathrm{a})\end{array}$ & Mouse & $1 / 600$ & & Synaptic Systems \\
\hline GlyR $\alpha 1$ subunit & $\begin{array}{l}\text { Monoclonal } \\
\text { (mAb2b) }\end{array}$ & Mouse & $1 / 800$ & & Synaptic Systems \\
\hline $\begin{array}{l}\text { GABAaR } \gamma 2 \\
\text { subunit }\end{array}$ & Polyclonal & $\begin{array}{l}\text { Guinea } \\
\text { Pig }\end{array}$ & $1 / 3000$ & & $\begin{array}{l}\text { Kindly provided by } \\
\text { Prof. Dr. Fritschy }\end{array}$ \\
\hline GluR6/7 subunit & $\begin{array}{l}\text { Monoclonal } \\
\text { (NL904) }\end{array}$ & Rabbit & $1 / 1000$ & & Millipore \\
\hline GluA2 & $\begin{array}{l}\text { Monoclonal } \\
(6 \mathrm{C} 4)\end{array}$ & Mouse & $1 / 1000$ & & Millipore \\
\hline GluN1 (NMDA- & Monoclonal & Mouse & $1 / 1000$ & & Synaptic Systems \\
\hline
\end{tabular}




\begin{tabular}{lllll}
\hline Receptor 1) & (M68) & & & \\
VIAAT & Polyclonal & Guinea & $1 / 500$ & Synaptic Systems \\
& & Pig & & \\
VGluT1 & Polyclonal & Guinea & $1 / 2000$ & Synaptic Systems \\
& & Pig & & \\
VGluT2 & Polyclonal & Guinea & $1 / 1000$ & Synaptic Systems \\
& & Pig & \\
\hline
\end{tabular}

\subsubsection{Secondary antibodies}

Table 2.2: Secondary antibodies used in this study

\begin{tabular}{llllll}
\hline Antigen & Conjugated dye & Host & IHC & Westerns Blots & Company \\
\hline Rabbit & Alexa fluor $488 \mathrm{~nm}$ or & Goat & $1 / 1200$ & & Molecular Probes \\
& $555 \mathrm{~nm}$ & & & & Molecular Probes \\
Mouse & Alexa fluor $488 \mathrm{~nm}$ or & Goat & $1 / 1200$ & & Molecular Probes \\
& $555 \mathrm{~nm}$ & & & & Invitrogen \\
Guinea Pig & Alexa fluor $488 \mathrm{~nm}$ or & Goat & $1 / 1200$ & & Rockland \\
\hline & $555 \mathrm{~nm}$ & & & $1 / 2000$ & $1 / 2000$ \\
\hline
\end{tabular}

\subsection{Electrophysiology}

\subsubsection{Preparation of acute slices}

Postnatal day 18-23 mice (P18-23) were anesthetized with Isofluorane (DeltaSelect) and decapitated. Brains were removed from the skull and placed into an ice-cold sucrosebased slicing solution (230 mM Sucrose, $26 \mathrm{mM} \mathrm{NaHCO}_{3}, 2 \mathrm{mM} \mathrm{KCl}, 1 \mathrm{mM} \mathrm{KH} \mathrm{OP}_{4}, 1 \mathrm{mM}$ $\mathrm{MgCl}_{2} \times 6 \mathrm{H}_{2} \mathrm{O}, 10 \mathrm{mM}$ Glucose, $0.5 \mathrm{mM} \mathrm{CACl}_{2}$ ) and oxygenated with carbogen gas. Axial brain sections (400 $\mu$ m thick) were obtained using a vibratome (Leica VT1200S). During the cutting procedure, the tissue was oxygenated with carbogen and immersed in ice-cold 
sucrose-based solution. The sections were transferred to a holding chamber and maintained under interface conditions with recording solution (ACSF: $120 \mathrm{mM} \mathrm{NaCl}, 26 \mathrm{mM} \mathrm{NaHCO} 3,1$ $\mathrm{mM} \mathrm{KH} \mathrm{PO}_{4}, 2 \mathrm{mM} \mathrm{KCl}, 2 \mathrm{mM} \mathrm{MgCl}_{2} \times 6 \mathrm{H}_{2} \mathrm{O}, 10 \mathrm{mM}$ Glucose, $2 \mathrm{mM} \mathrm{CaCl}$ ). Before recording, sections were allowed to recover for $1 \mathrm{~h}$.

\subsubsection{Gamma oscillations under interface conditions}

After slicing, brain sections were kept floating on the surface of the extracellular recording solution saturated with carbogen gas. Network activity was evaluated by recording extracellular field potentials. Hippocampal slices were placed in an interface recording chamber (BSC-BU Base Unit with the BSC-HT Haas Top, Harvard Apparatus). Slices were placed on a nylon mesh at the interface of a stream of extracellular recording solution and a stream of warm, moist carbogen gas. The temperature of the stream was controlled to be at $33^{\circ} \mathrm{C}$. Extracellular recording electrodes with a resistance between 1.5-2.5 $\mathrm{M} \Omega$ were filled with extracellular recording solution and placed in the hippocampal pyramidal cell layers of CA3 and CA1. Extracellular field potentials were recorded with a 700B amplifier (Axon, Molecular Devices) and the Digidata 1440 A data acquisition system (Axon, Molecular Devices). During the recordings, a Bessel filter at $3 \mathrm{kHz}$ and a gain of 100 was applied. To induce gamma oscillations, $100 \mathrm{nM}$ kainate was applied in extracellular recording solution. Oscillatory activity could be recorded 10 min after the start of kainate application. For each slice, baseline field potentials were recorded for 30 min during ACSF application, followed by recording of gamma-oscillation field-potentials during application of ACSF $+100 \mathrm{nM}$ kainate for $30 \mathrm{~min}$. Data were analyzed using Axograph X software (Axon Instruments). Traces were filtered at $100 \mathrm{kHz}$ and the power spectrum was calculated for 10-min epochs (last $10 \mathrm{~min}$ of each recording) of recorded field activity. The baseline power spectrum was subtracted from the power spectrum induced by kainate. Frequency and the power of the maximum peak were evaluated. The value determined by the peak in the power spectrum was calculated according to the gamma oscillatory frequency band $(25-45 \mathrm{~Hz})$.

\subsection{Statistics}

Statistical analyses were performed using unpaired, two-tailed Student's T-test. Variance is expressed as the standard error of the mean. 


\section{Results}

\subsection{Optimization of NL4 immunohistochemistry}

In order to detect NL4 in the CNS, we generated an anti-NL4 rabbit polyclonal antibody. The antibody is targeted against the extracellular fragment of NL4 between the esterase domain and the transmembrane domain (Jamain et al., 2008). The specificity of the antibody was tested by comparing the labeling obtained from WT brain and spinal cord sections with corresponding samples from the NL4 deletion mutant. A detection threshold was set for each brain region in order to eliminate any remaining non-specific background as seen in the NL4-KO mice. Using this procedure, conditions were obtained in which immunohistochemical NL4 staining was reproducibly observed in the different brain regions of the WT but not in the NL4-KO control (see methods Materials and Methods, section 2.2). As illustrated in Figure 3.1, the antibody conditions used for this project allowed for specific and reproducible staining in the WT that was not detected in the KO sections (Figure 3.1 AC). 


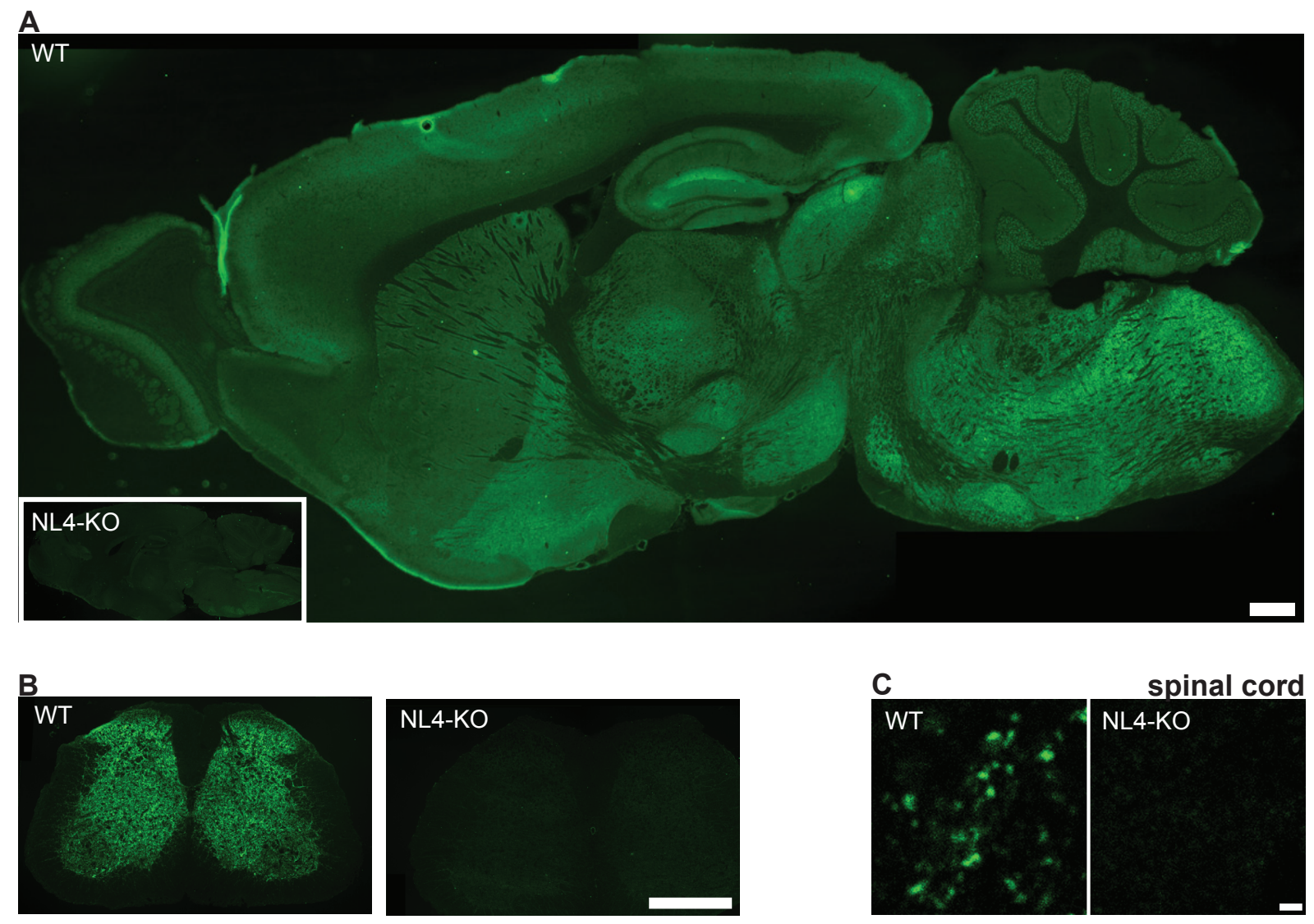

Figure 3.1: Immunoreactivity of neuroligin 4 in the brain (A) and the spinal cord (B-C). (A) Sagittal section of an adult mouse brain immunostained for NL4; showing that NL4 is widely immunoreactive throughout the brain. NL4 staining is more intense in some areas e.g. the brainstem. This staining is absent in the NL4-KO mouse (inset). (B) NL4 is also immunoreactive in the spinal cord, except for the most superficial laminae of the dorsal horn. Again, NL4 staining is absent in NL4-KO. (C) Under high magnification, punctate NL4 staining is observed in the spinal cord, reminiscent of the staining pattern observed with classical synaptic labeling. Puncta observed in WT mice are absent in NL4-KO mice, indicating specific NL4 staining.

Scale bars: $\mathrm{A}$ and $\mathrm{B}=500 \mu \mathrm{m}, \mathrm{C}=1 \mu \mathrm{m}$

\subsection{Distribution of neuroligin 4 in the brain}

NL4 mapping is a crucial step in understanding the role and function of the protein in the CNS. In the mouse CNS, NL4 is detected in spinal cord, superior colliculus, thalamus, substantia nigra, brainstem, hippocampus, and cortex at different degrees of intensity (Figure 3.1A). In contrast, no specific staining was detected in the olfactory bulb, cerebellum (Figure 3.1A) and amygdala (Figure 3.2D). The highest labeling of NL4 in the brain is observed in the brainstem (Figure 3.1A). The protein is widely immunoreactive in the spinal cord within laminae II to X (Figure 3.1B). At high magnification, NL4 labeling appears to be clustered in the brainstem (Figure 3.1C). 

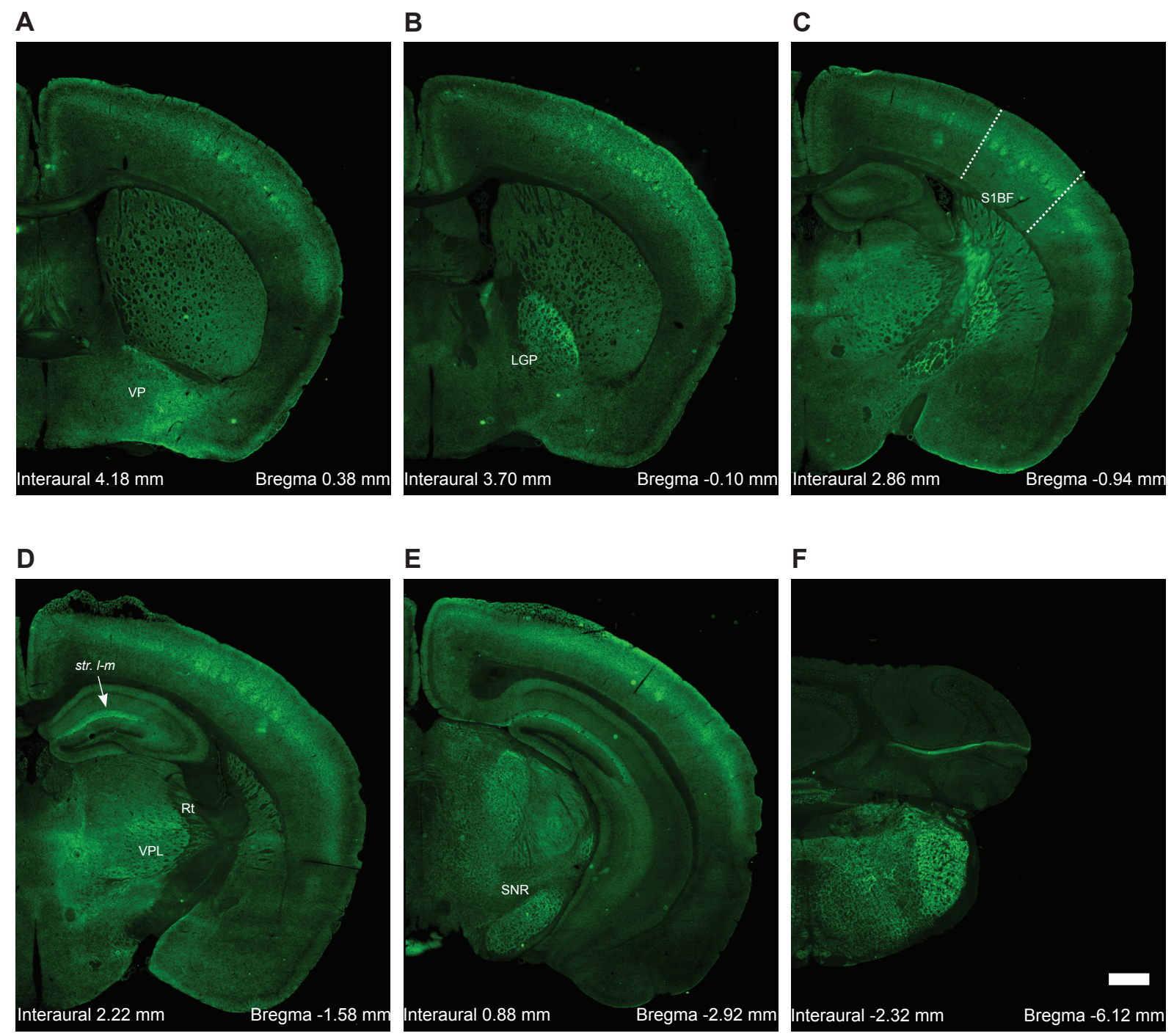

Figure 3.2: NL4 distribution in coronal brain sections. (A-E) NL4 labeling is detectable in the cortical layer IV with increased intensity in the primary somatosensory cortex that corresponds to the barrel field (B-D). NL4 is present in the basal ganglia, and specifically in the ventral pallidum (A), globus pallidus (B) and the substantia nigra (E). In the hippocampus (D), NL4 is present in CA1, more precisely in the stratum lacunosum moleculare. The strongest staining intensity was found in the brainstem (F), where NL4 is widely immunoreactive.

Abbreviations: VP, ventral pallidum; LGP, lateral globus pallidus; S1BF, primary somatosensory cortex barrel field; str. 1-m, CA1 stratum lacunosum moleculare; Rt, reticular thalamic nucleus; VPL, ventral posterior nucleus; SNR, substantia nigra reticular part. Interaural and Bregma refer to the stereotaxic coordinate of each brain (Franklin, 2001).

Scale bar $=500 \mu \mathrm{m}$

NL4 protein immunoreactivity differs depending on the specific brain region examined (Figure 3.2). In the cortex, NL4 is localized primarily to layer IV, with a particularly prominent staining in the barrel field in layer IV of the primary somatosensory cortex (Figure 3.2A-D). In the hippocampus, NL4 is labeled mainly in a specific layer of CA1, the stratum lacunosum moleculare (str. l-m) (Figure 3.2D). Interestingly, NL4 is also 
localized to a group of nuclei that belong to the basal ganglia; indeed the protein is immunoreactive in the ventral pallidum (VP) (Figure 3.2A), lateral globus pallidus (LGP) (Figure 3.2B-C) and substantia nigra (SNR) (Figure 3.2E). NL4 protein is also present in some thalamic nuclei such as the reticular thalamic nucleus (Rt) and the ventral posterior nucleus (VPL) (Figure 3.2C-D). In the brainstem, NL4 labeling is ubiquitous (Figure 2F) and has the strongest staining intensity compared to the rest of the brain (Figure 1A).

\subsubsection{Specific synaptic localization of NL4}

Earlier studies have shown that in the brain, the NL1 isoform is specifically localized at excitatory postsynapses (Song et al., 1999) and the NL2 isoform is specifically localized at inhibitory postsynapses (Varoqueaux et al., 2004). However, the synaptic localization of NL4 remains to be defined, and this knowledge is necessary to understand the function of the protein.

For our synaptic localization experiments, four different brain regions were chosen according to the NL4 staining specificity and intensity, the types of synapses present and the potential correlation to behavioral deficits relevant to autism. (1) The barrel cortex was selected for the specificity of the staining, and because it is a useful model system to investigate plasticity in primary sensory cortex. (2) The CA1 str. l- $m$ of the hippocampus was selected for the specific NL4 labeling, the well-studied synaptic architecture and the role in behaviors such as memory consolidation. Finally, (3) the globus pallidus and (4) the brainstem were selected because they both strongly express NL4 and mainly contain inhibitory synapses, and recent work showed that NL4 plays a functional role at glycinergic synapses in the retina (Hoon et al., 2011).

A series of colocalization experiments were carried out with postsynaptic scaffolding proteins to determine if NL4 is specifically segregated to certain synaptic subtypes.

Gephyrin was used as a marker for inhibitory postsynapses and PSD-95 as a marker for excitatory postsynapses. For the majority of the brain regions, the global pattern of NL4 immunoreactivity resembled that of gephyrin labeling, indicating inhibitory synaptic localization (Figure 3.3). 

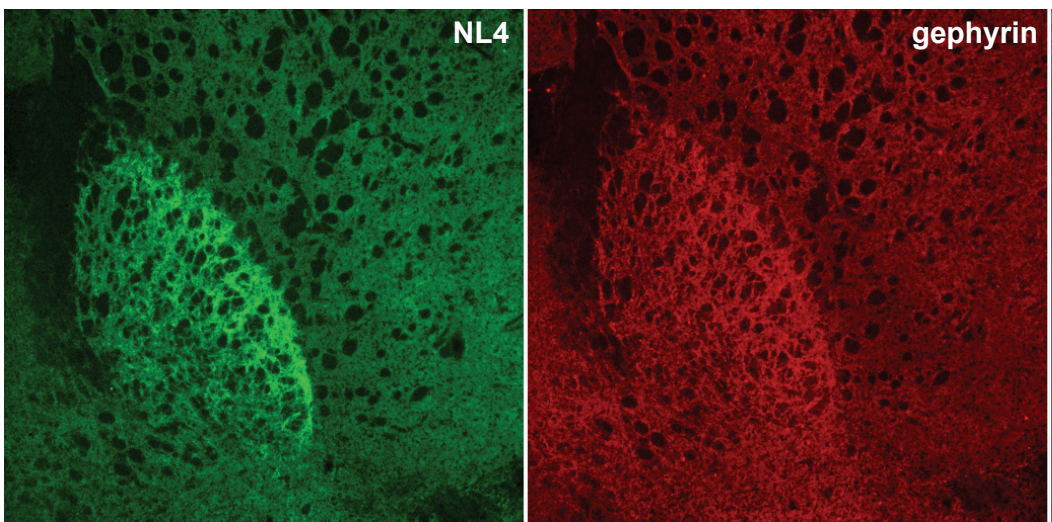

B

somatosensory primary cortex
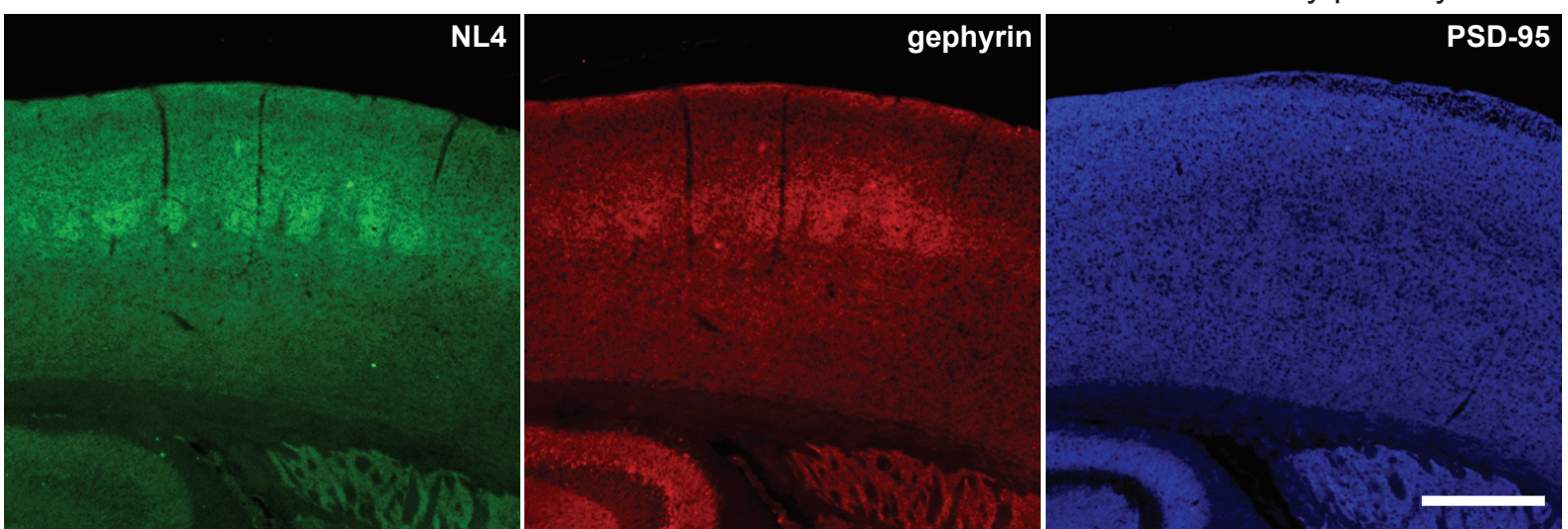

C

hippocampus

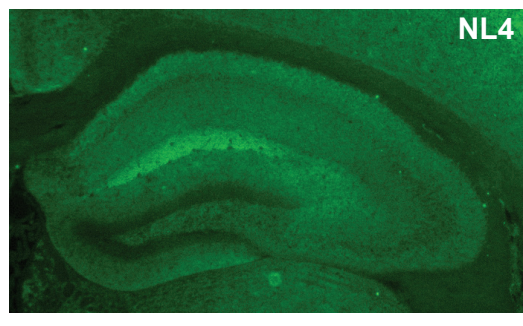

NL4

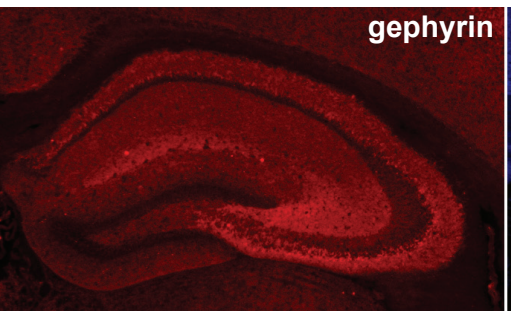

gephyrin
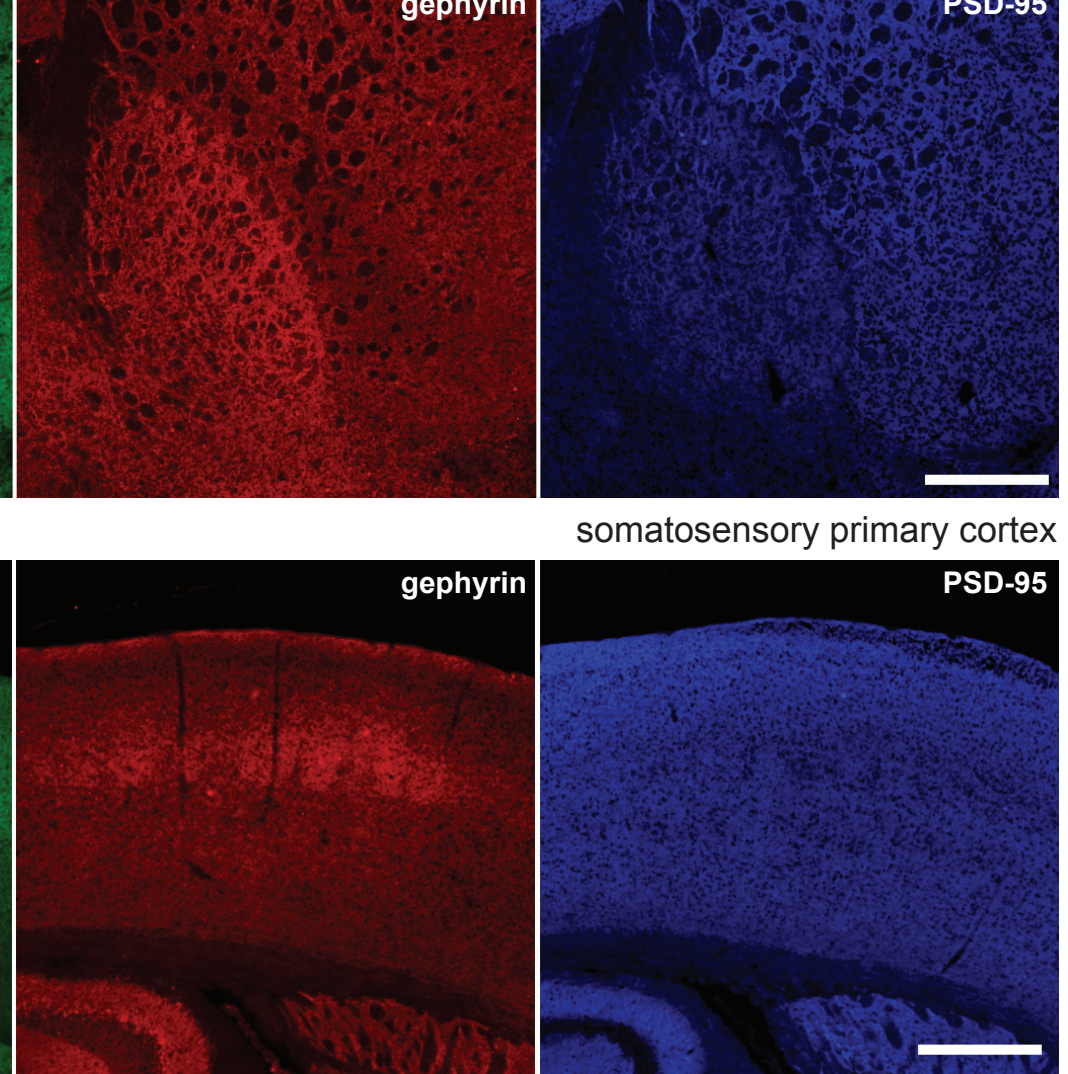

D

brainstem

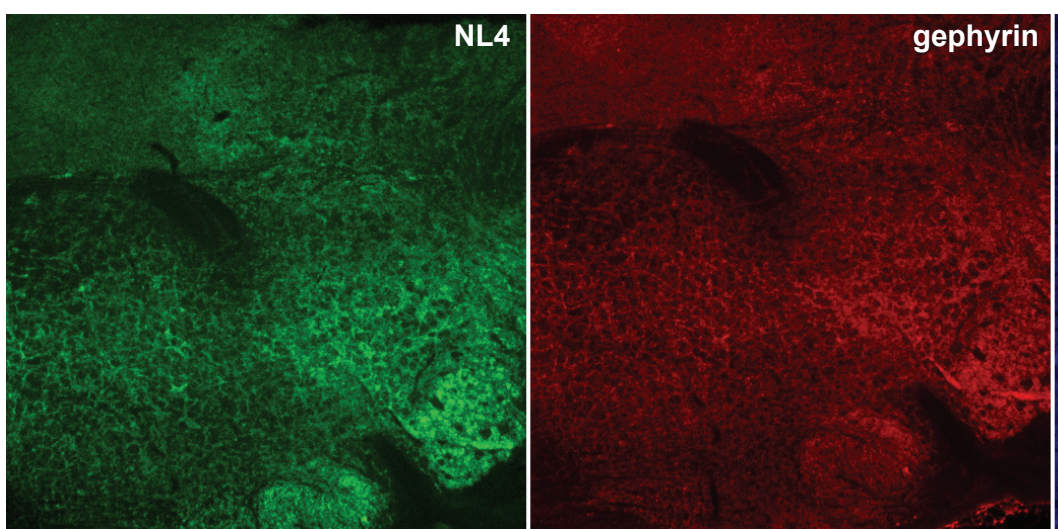

Figure 3.3: NL4 localization matches gephyrin labeling. Comparison of immunofluorescence detection of NL4 (green) with gephyrin (GABAergic postsynaptic marker) (red) and PSD-95 localization (glutamatergic postsynaptic marker) (blue). (A) NL4 immunoreactivity in the globus pallidus matches that of gephyrin, indicating that NL4 is immunoreactive preferentially in the same regions as gephyrin. The PSD-95 labeling in this region is weaker compared to the caudate putamen. (B) In the cortex, PSD-95 labeling is 
ubiquitous in the entire cortical layers, whereas gephyrin and NL4 labeling is observed predominantly in the barrel cortex. (C) The CA1 stratum lacunosum moleculare strongly contains NL4 and gephyrin, while the same layer in CA3 contains only gephyrin. PSD-95 is strongest in CA1 stratum oriens and radiatum. (D) In the whole brainstem, NL4 and gephyrin labeling also match in terms of intensity and localization, whereas PSD-95 staining is weaker. Scale Bar: $500 \mu \mathrm{m}$

In the globus pallidus (Figure 3.3A), the NL4 labeling matched precisely with the gephyrin localization. In contrast, the PSD-95 labeling was weaker in this region, especially compared to the caudate putamen, which strongly contains the excitatory synapse scaffolding protein. In the layer IV of the primary somatosensory cortex (Figure 3.3B), gephyrin and NL4 are labeled in the barrel field, whereas PSD-95 immunoreactivity is present in all cortical layers. In this area, NL4 labeling overlaps with both PSD-95 and gephyrin staining. In the hippocampus (Figure 3.3C), the NL4 localization did not correspond with the strongest PSD95 labeling, which is higher in the str. or and str. rad of CA1, whereas NL4 is weakly but widely labeled in the whole hippocampus with the strongest intensity in CA1 str. l-m. The NL4 immunoreactivity matches with that of gephyrin staining, but interestingly, gephyrin is labeled in the CA3 str. $l-m$ whereas NL4 is not. In the brainstem (Figure 3.3D), NL4 shares the same pattern of immunoreactivity as gephyrin. PSD-95 labeling was found to be relatively weak in the whole brainstem compared to the forebrain areas. Some sparse immunoreactivity was found in the brainstem, but this did not colocalize with the NL4 labeling.

Having observed colocalization between NL4 and gephyrin in all brain regions studied, it was crucial to know whether NL4 was present at inhibitory synapses and therefore colocalized with gephyrin at single synapses. Here, high magnification images of single synaptic density clusters or puncta were captured using the same postsynaptic scaffolding proteins as markers as described above.

The first observation at this magnification (63X zoom 8$)$ was that the pattern of NL4 labeling differs noticeably from one brain region to the other. Indeed, in the barrel cortex and the hippocampus (Figure 3.4A and B), the staining is much more diffuse and corresponds more to small puncta rather than dense clusters. In contrast, in the brainstem and the globus pallidus (Figure 3.4C and D) the NL4 staining corresponds to dense dispersed clusters.

In the barrel cortex (Figure 3.4A), 72\% $\pm 11.51 \%$ of NL4 puncta colocalize with PSD-95, whereas only $29.45 \% \pm 5.7 \%$ of the NL4 clusters are localized with the gephyrin puncta. Gephyrin and NL4 in the somatosensory cortex are both localized specifically in layer IV of 
cortex, whereas the PSD-95 staining was found in all the cortical layers (Figure 3.3A). Thus, the overlap of NL4 and gephyrin immunoreactivity at the global level does not necessarily predict a colocalization at the synaptic level, emphasizing the importance of NL4 localization at this level.

\section{A barrel cortex}
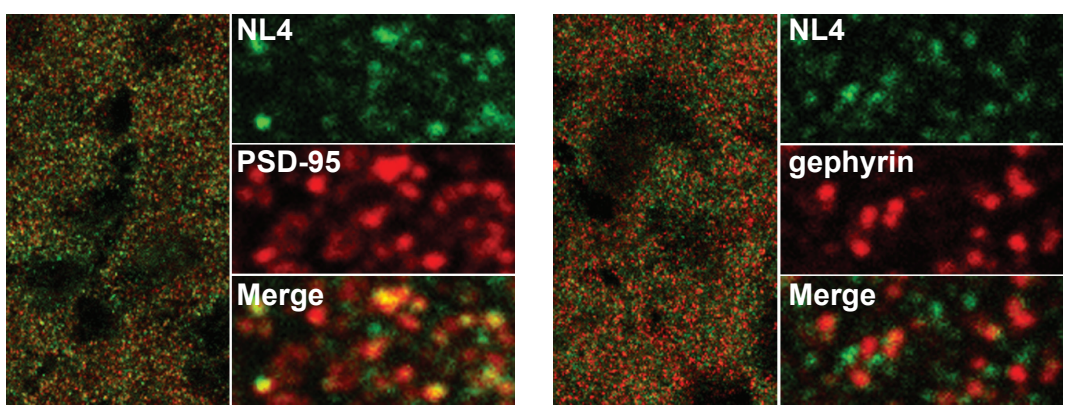

\begin{tabular}{|c|c|c|}
\hline $\begin{array}{c}\text { NL4 colocalized in the bar- } \\
\text { rel Cortex with }\end{array}$ \\
\hline & PSD-95 & gephyrin \\
\hline$\%$ & $\mathbf{7 2 . 3 5}$ & $\mathbf{2 9 . 4 5}$ \\
\hline SEM & 11.51 & 5.7 \\
\hline
\end{tabular}

B

hippocampus CA1 str. I-m
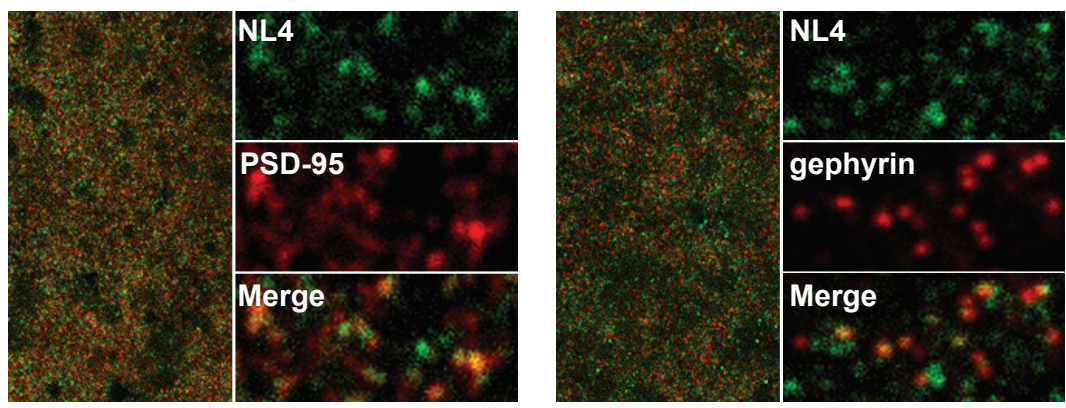

\begin{tabular}{|c|c|c|}
\hline \multicolumn{3}{|c|}{$\begin{array}{c}\text { NL4 colocalized in the str. } \\
\text { I- } \boldsymbol{m} \text { with }\end{array}$} \\
\hline & PSD-95 & gephyrin \\
\hline \% & $\mathbf{4 8 . 6 4}$ & $\mathbf{5 5 . 3 2}$ \\
\hline SEM & 4.52 & 9.89 \\
\hline
\end{tabular}

C globus pallidus
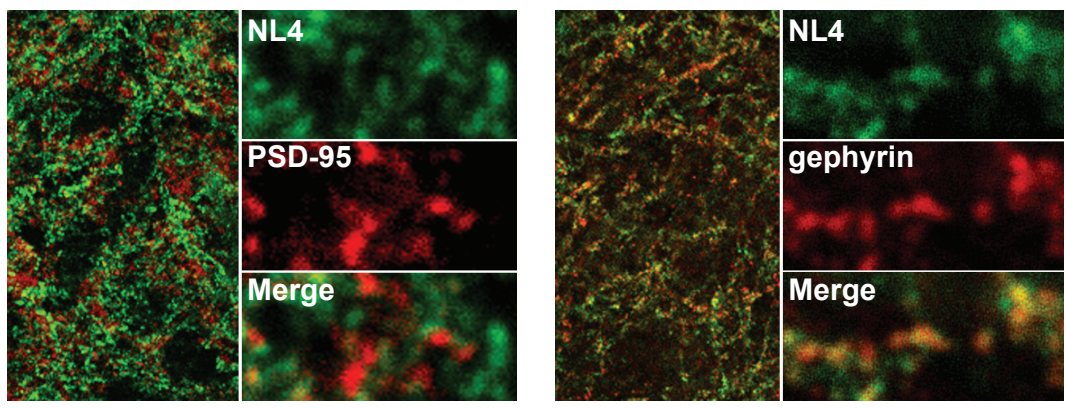

\begin{tabular}{|c|c|c|}
\hline \multicolumn{3}{|c|}{$\begin{array}{c}\text { NL4 colocalized in the glo- } \\
\text { bus pallidus with }\end{array}$} \\
\hline & PSD-95 & Gephyrin \\
\hline$\%$ & $\mathbf{1 4 . 9 7}$ & $\mathbf{7 4 . 1 8}$ \\
\hline SEM & 6.45 & 6.02 \\
\hline
\end{tabular}

D brainstem
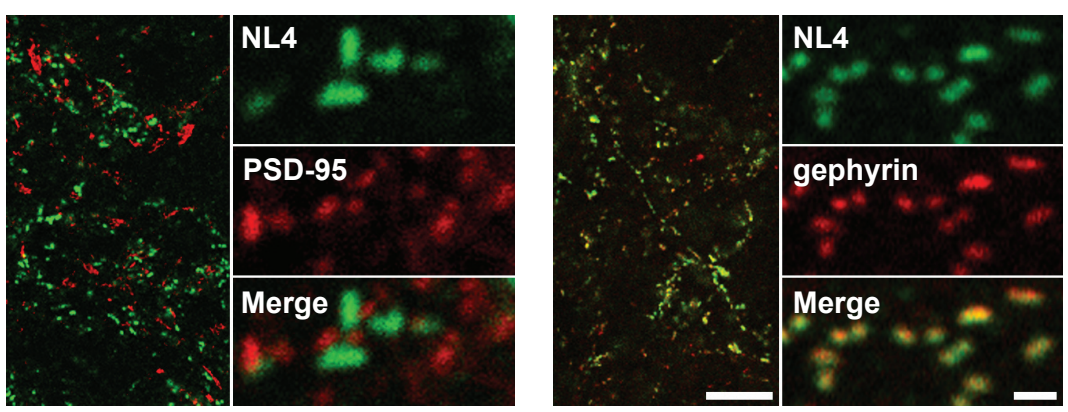

\begin{tabular}{|c|c|c|}
\hline \multicolumn{3}{|c|}{$\begin{array}{c}\text { NL4 colocalized in the } \\
\text { brainstem }\end{array}$} \\
\hline & PSD-95 & gephyrin \\
\hline$\%$ & $\mathbf{1 8 . 9 4}$ & $\mathbf{7 9 . 0 8}$ \\
\hline SEM & 2.82 & 0.92 \\
\hline
\end{tabular}

Figure 3.4: NL4 is associated with excitatory and inhibitory synapses depending on the brain region. Confocal images showing colocalization of NL4 (green) immunoreactivity in four different brain regions with PSD-95 (glutamatergic postsynaptic marker) (red) or 
gephyrin (GABAergic postsynaptic marker) (red). Tables on the right show quantification of the degree of colocalization, obtained by evaluating the NL4 clusters (set by a threshold) for colocalization with PSD-95 or gephyrin (set by a threshold) ( $\mathrm{n}=4$ pairs). (A) NL4 is present at excitatory postsynapses in the barrel cortex but not in the others brain regions. (B) NL4 is colocalized with PSD-95 and with gephyrin in the hippocampus. (C-D) NL4 is present at inhibitory postsynapses in the globus pallidus and the brainstem.

Scale bars: overview $=10 \mu \mathrm{m}$; detail $=1 \mu \mathrm{m}$

Interestingly, in the CA1 str. $l-m$ of the hippocampus, NL4 appears to be colocalized with both PSD-95 and gephyrin, indeed $48.64 \% \pm 4.52 \%$ of the NL4 puncta colocalize with PSD-95 and 55.32\% $\pm 9.89 \%$ with gephyrin. This indicates that NL4 protein in that particular region is localized at excitatory and inhibitory synapses (Figure 3.4B).

In the globus pallidus (Figure 3.4C), where the NL4 labeling is clustered, $74.18 \% \pm$ $6.02 \%$ of NL4 clusters colocalize with gephyrin. On the other hand, only $14.97 \% \pm 6.45 \%$ of NL4 puncta in the globus pallidus are colocalized with PSD-95.

In the brainstem (Figure 3.4D), 79.08\% $\pm 0.92 \%$ of NL4 puncta are localized with gephyrin, whereas only $18.94 \% \pm 2.82 \%$ of the puncta are localized with PSD-95. Gephyrin immunolabeling corresponds to that of NL4 in the globus pallidus (Figure 3.3A) and the brainstem (Figure 3.3D). It should be noted that both brain regions weakly express PSD-95, and therefore the analysis was carried out with relatively few PSD-95 puncta.

These data indicate that NL4 is present at both excitatory and inhibitory synapses, and demonstrates for the first time that NL4 may not only be present at inhibitory synapses, but also at excitatory synapses in specific brain regions.

\subsubsection{Characterization of NL4-containing synapses}

Having established that NL4 is colocalized with gephyrin and PSD-95 at inhibitory and excitatory postsynapses, the next step was to identify the pre- and post-synaptic machinery present at the NL4-positive synapses. Identification of the exact composition of the NL4-containing synapses is an essential step to understand the function of the protein.

Utilizing a series of antibodies (Table 2.1), the characterization of NL4-positive synapses was performed in the brain regions that were already mentioned above. 
The presynaptic element was identifiable by the presence of vesicle transporters which carry neurotransmitters into vesicles. The excitatory presynaptic staining was carried out using anti-VGluT1 and anti-VGluT2 antibodies. These antibodies showed a complementary degree of labeling, since the two transporters mark specific separate synaptic input pathways in several of the brain regions under study such as the barrel cortex (Liguz-Lecznar and Skangiel-Kramska, 2007). To label the inhibitory presynaptic side, an anti-VIAAT antibody was used.

In order to identify if NL4 was present at AMPAR, NMDAR or GABAR-containing synapses, receptor-specific immunohistochemistry was carried out. Specifically, AMPAR were labeled using an antibody raised against the subunit GluA2, and NMDAR were labeled with an antibody targeted against the GluN1 subunit. An antibody raised against the subunit $\gamma 2$ of the $\mathrm{GABA}_{A} \mathrm{R}$ was used to stain $\mathrm{GABA}_{\mathrm{A}} \mathrm{R}$, as the $\gamma 2$ subunit is a core component of a majority of $\mathrm{GABA}_{A} \mathrm{R}$ (Fritschy and Mohler, 1995) and is required for $\mathrm{GABA}_{\mathrm{A}} \mathrm{R}$ clustering at the synapse (Essrich et al., 1998).

In the following three sections, NL4 synaptic subtype is described according to the synaptic markers with which the protein colocalizes.

\subsubsection{NL4 is present at inhibitory synapses in the globus pallidus and brainstem}

In the globus pallidus, NL4 is ubiquitously immunoreactive (Figure 3.3). A series of colocalization experiments to determine if NL4 in this region is segregated to a synaptic subtype were carried out. NL4 was not observed to be apposing the excitatory presynaptic marker VGluT2 (11.7\%) (Figure 3.5B-C). It is important to note that no VGluT1 immunoreactivity was detected in the entire globus pallidus. At the postsynaptic side, NL4 was not observed to be colocalized with GluA2 (10.08\%) or GluN1 (8.08\%) (Figure 3.5E-F). These two observations support the notion that in the globus pallidus, NL4 is absent from excitatory synapses. NL4 was more apposed to VIAAT $(27.89 \% \pm 6.54 \%)$ (Figure 3.5A) than VGluT2 $\left(11,7 \% \pm 0.91 \%\right.$ ) (Figure 3.5C), and NL4 colocalizes extensively with the $\mathrm{GABA}_{\mathrm{A}} \mathrm{R}$ subunit $\gamma 2(71.01 \% \pm 4.97 \%)$ (Figure 3.5D). Together with the gephyrin experiments in which NL4 extensively colocalized with this inhibitory synapse marker (Figure 3.4C), these results further indicate the presence of NL4 at inhibitory postsynapses in the globus pallidus. 
In the brainstem, the region with the highest NL4 staining intensity (Figure 3.1A), NL4 staining was observed as big clusters (Figure 3.6). In this region NL4 is also mainly apposed to VIAAT $(48.01 \% \pm 2.92 \%$ ) (Figure $3.6 \mathrm{~A}$ ) as compared to VGluT1 $(8.39 \% \pm$ $4.11 \%$ ) (Figure 3.6B) and VGLuT2 $(19.82 \% \pm 5.45 \%$ ) (Figure 3.6C). At the postsynaptic side, NL4 was found mainly to be present at $\mathrm{GABA}_{\mathrm{A}} \mathrm{R}$ clusters $(55.6 \% \pm 5.66 \%)$ (Figure 3.6D) and almost no colocalization was observed with AMPAR $(5.26 \% \pm 0.62 \%)$ (Figure 3.6E) and NMDAR $(9.75 \% \pm 2.24 \%$ ) (Figure 3.6F). These data support the notion that NL4 is only present at inhibitory synapses in the brainstem. 
A
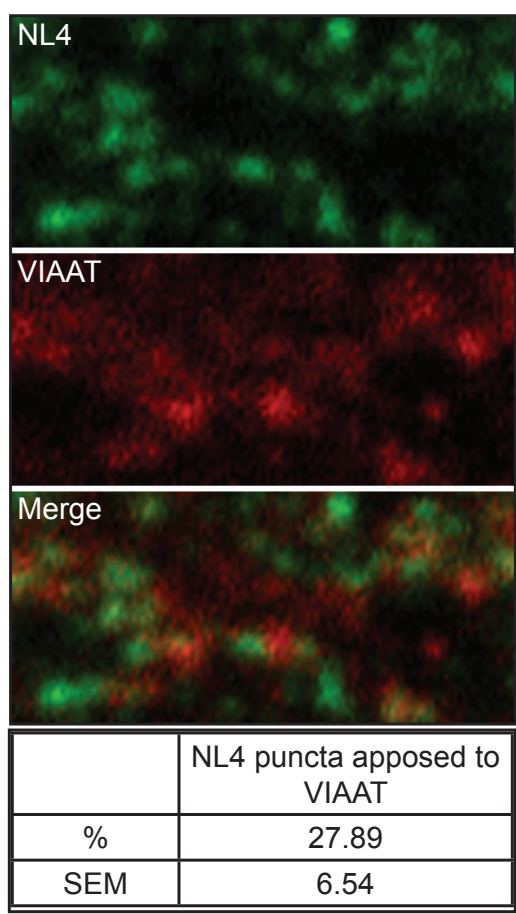

D

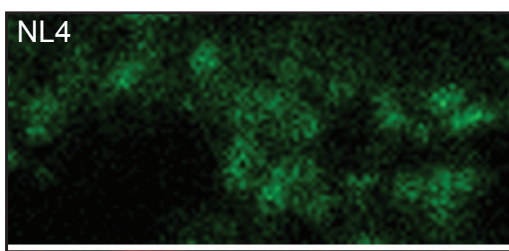

$\mathrm{GABA}_{\mathrm{A}} \mathrm{R} \gamma 2$
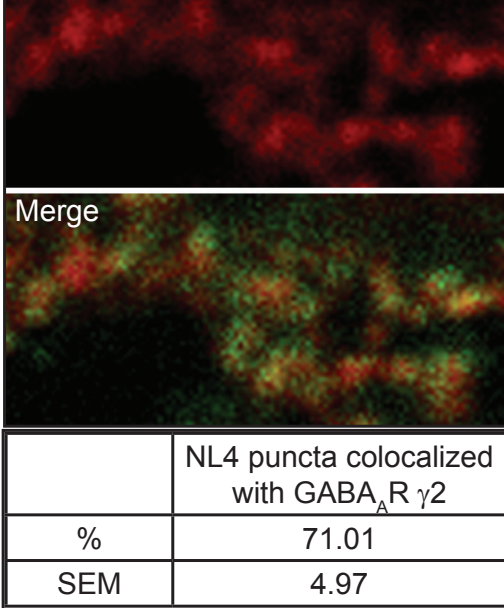

B
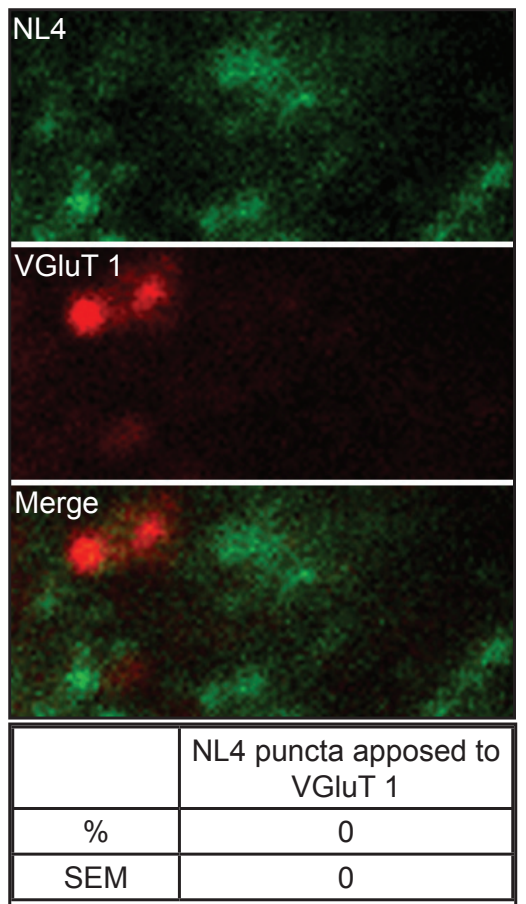

E
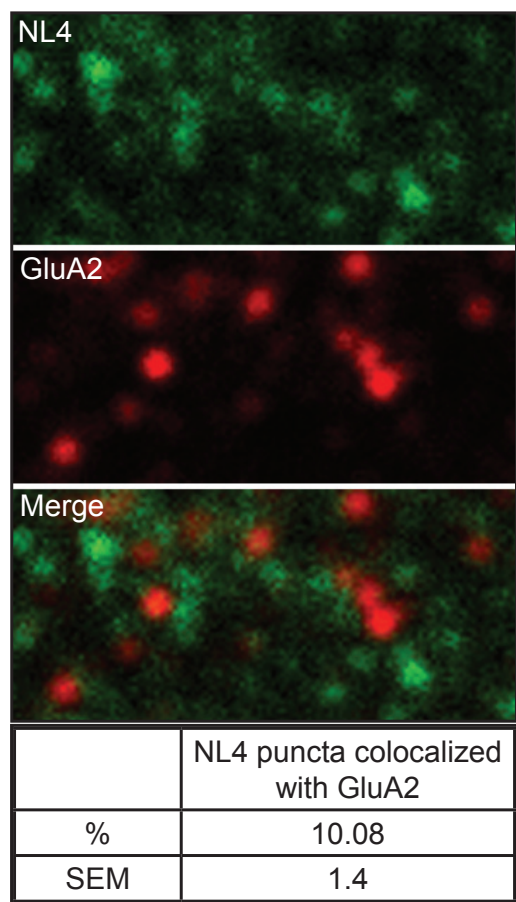

C

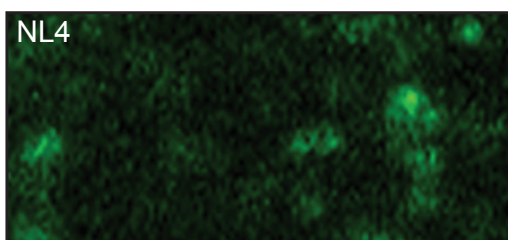

\section{VGIUT 2}

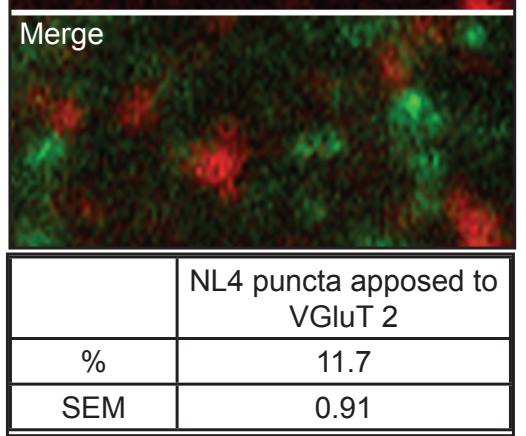

$\mathbf{F}$

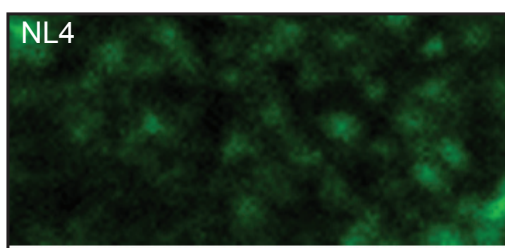

\section{GluN1}

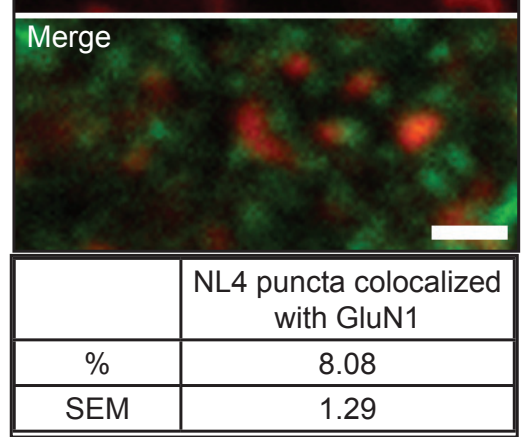

Figure 3.5: Localization of NL4 in the globus pallidus. To further characterize the distribution of NL4 in the globus pallidus, immunolabelings were carried out for NL4 together with inhibitory (VIAAT) (A) and excitatory (B, C) (VGluT1 and 2) presynapse markers, as well as with inhibitory $\left(\mathrm{GABA}_{\mathrm{A}} \mathrm{R} \gamma 2\right.$ for the $\left.\mathrm{GABA}_{\mathrm{A}} \mathrm{R}\right)(\mathrm{D})$ and excitatory (GluA2 for AMPAR and GluN1 for the NMDAR) (E, F) postsynapse markers. Tables below figures show quantification of the degree of colocalization, obtained by evaluating NL4 clusters (set by a threshold) and their colocalization with the different synaptic markers tested (set by a threshold) ( $\mathrm{n}=3$ pairs). In the globus pallidus, NL4 is mainly apposed to VIAAT on the 
presynaptic side. At the postsynaptic side, NL4 colocalizes extensively with the ubiquitous $\mathrm{GABA}_{\mathrm{A}}$ receptor subunit $\mathrm{GABA} \mathrm{A} \gamma 2$.

Scale bars: overview $=10 \mu \mathrm{m}$; detail $=1 \mu \mathrm{m}$

A
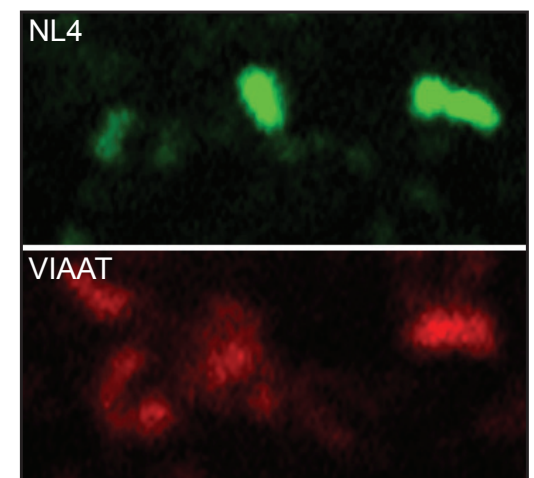

Merge

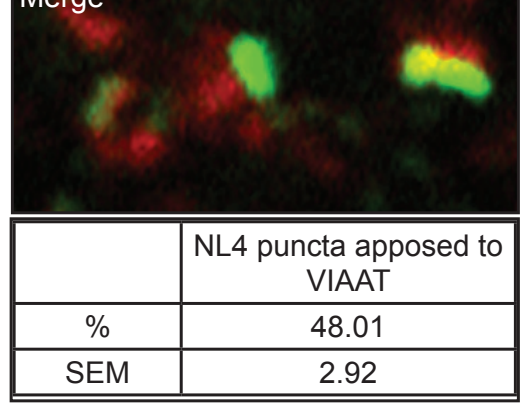

D
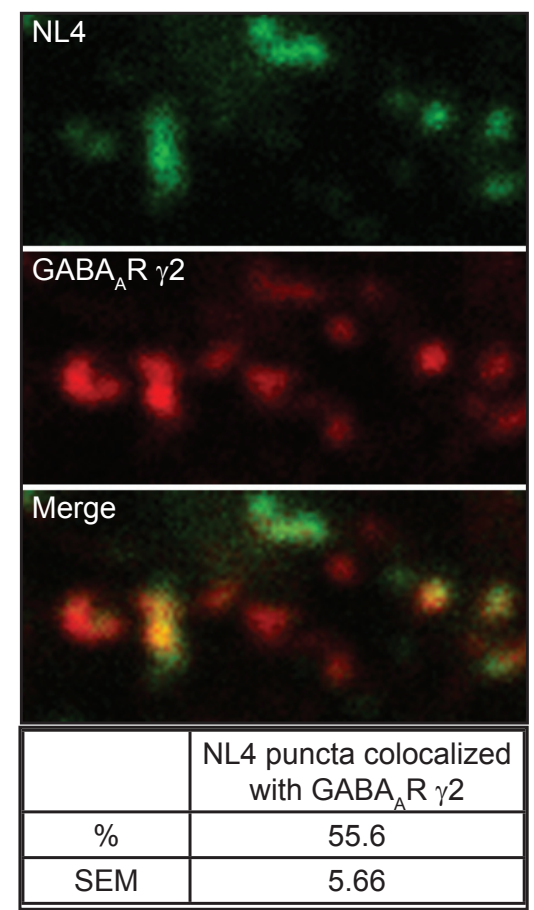

B
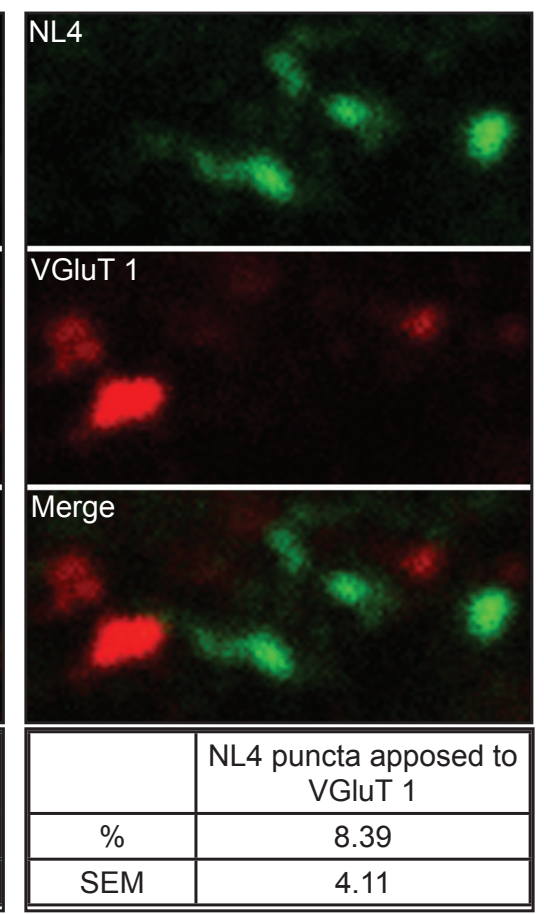

$E$
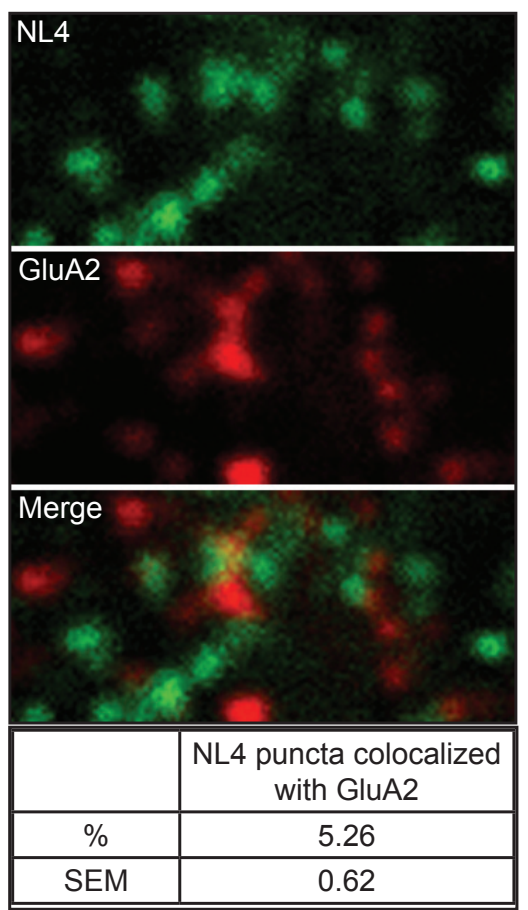

C
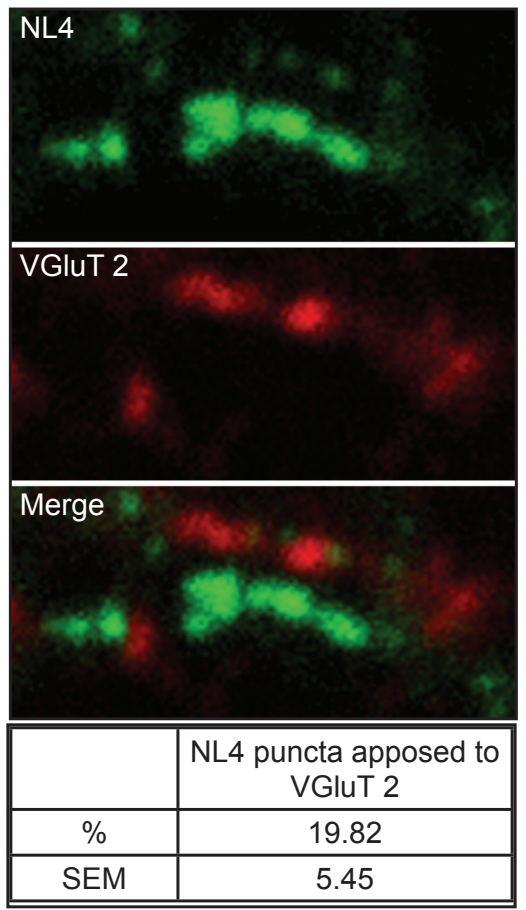

$\mathbf{F}$
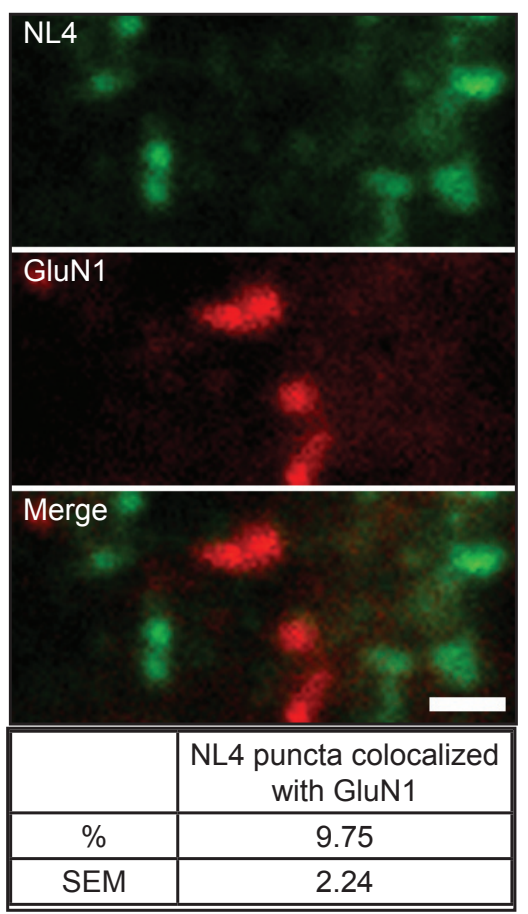

Figure 3.6: Localization of NL4 in the brainstem. To further characterize the distribution of NL4 in the brainstem, immunolabelings were carried out for NL4 together with inhibitory (VIAAT) (A) and excitatory (B, C) (VGluT1 and 2) presynapse markers, as well as with 
inhibitory $\left(\mathrm{GABA}_{\mathrm{A}} \mathrm{R} \gamma 2\right.$ for the GABA $\mathrm{A}$ ) (D) and excitatory (GluA2 for AMPAR and GluN1 for the NMDAR) (E, F) postsynapse markers. Tables below figures show quantification of the degree of colocalization, obtained by evaluating NL4 clusters (set by a threshold) and their colocalization with the different synaptic markers tested (set by a threshold) ( $n=3$ pairs). In the brainstem, NL4 is mainly apposed to VIAAT on the presynaptic side. At the postsynaptic side, NL4 colocalizes extensively with the ubiquitous $\mathrm{GABA}_{\mathrm{A}}$ receptor subunit $\mathrm{GABA}_{\mathrm{A}} \mathrm{R} \gamma 2$.

Scale bars: overview $=10 \mu \mathrm{m}$; detail $=1 \mu \mathrm{m}$

Remarkably, the percentage of NL4 puncta that colocalize with $\mathrm{GABA}_{\mathrm{A}} \mathrm{R}$ was found to be higher in the globus pallidus $(71.01 \% \pm 4.97 \%$ ) (Figure 3.5D) than in the brainstem $(55.6 \% \pm 5.66 \%)$ (Figure 3.6D). Unlike in the globus pallidus, inhibitory neurotransmission in the brainstem is mediated by both GABA and glycine synapses. In those CNS regions, both GABA and glycine can be released by the same synapses (Smith et al., 2000). Therefore, an additional question regarding the brainstem was to what extent NL4 is localized at glycinergic synapses. To identify glycinergic synapses, immunohistochemistry for the glycine receptor (GlyR) was carried out. GlyR are pentameric ionotropic receptors that have four $\alpha$ subunits and a single $\beta$ subunit. In the brainstem, NL4 is present at a major fraction of the $\alpha 1$ subunitcontaining GlyR (Figure 3.7A). Triple labeling for NL4, GABA $R$ and GlyR revealed that in the brainstem, NL4 is mainly colocalized with the GlyR and less with the GABA $\mathrm{R}$ (Figure 3.7B).

A

B

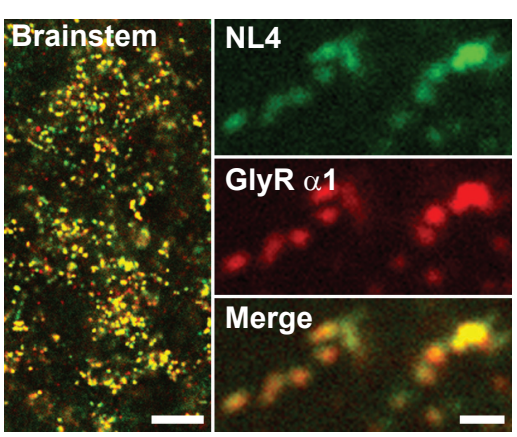

NL4 $\quad$ GABA $_{A} \mathbf{R} 2 \quad$ GlyR $\alpha 1$

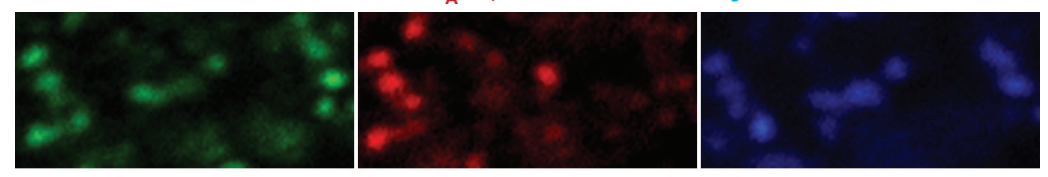

NL4 - GABA ${ }_{A}$ 2 NL4 - GlyR $\alpha 1 \quad$ NL4 - GABA ${ }_{A} R \gamma 2$ - GlyR

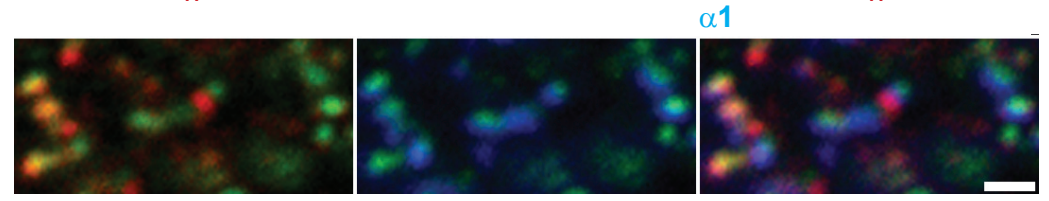

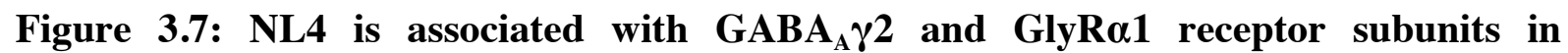
brainstem. Confocal images showing NL4 immunoreactivity in brainstem coronal sections. (A) Combined immunofluorescence detection of GlyR $\alpha 1$ subunit (red) and NL4 (green) shows that NL4 colocalizes extensively with GlyR $\alpha 1$ clusters, one of the subunits of the glycine receptor in the brainstem. (B) Combined immunofluorescence detection of $\mathrm{GABA}_{\mathrm{A}} \mathrm{R} \gamma 2$ (red) and GlyR $\alpha 1$ subunit (blue) with NL4 (green) shows that NL4 colocalizes with $\mathrm{GABA}_{\mathrm{A}}$ receptor and glycine receptor with a preference for the glycine receptor in the brainstem.

Scale bars: $(\mathrm{A})$ overview $=10 \mu \mathrm{m} ;(\mathrm{A}, \mathrm{B})$ detail $=1 \mu \mathrm{m}$ 
In summary, NL4 is present at inhibitory synapses in the brainstem and globus pallidus. The protein was found to be localized preferentially to glycinergic synapses in the brainstem but in the absence of glycinergic synapses, as in the globus pallidus, NL4 preferentially colocalizes with $\mathrm{GABA}_{\mathrm{A}} \mathrm{R}$.

\subsubsection{NL4 is present at excitatory synapses in the barrel cortex}

In the barrel cortex, NL4 labeling was observed as faint puncta (Figure 3.8). NL4 was mostly apposed to VGluT1 $(41.49 \% \pm 12.04 \%$ ) (Figure $3.8 \mathrm{~B})$ as compared to VGluT2 $(10.25 \% \pm 1.32 \%)$ (Figure $3.8 \mathrm{C}$ ) or VIAAT $(8.54 \% \pm 2.58 \%$ ) (Figure 3.8A). Together, these results from the presynaptic marker apposition experiments indicate that NL4 in the barrel cortex is present at excitatory synapses. However, colocalization experiments with postsynaptic markers did not emphasize the presence of NL4 with any of the main synaptic receptors. Only $22.78 \% \pm 4.67 \%$ of the NL4 puncta are localized with $\mathrm{GABA}_{\mathrm{A}} \mathrm{R}$ (Figure 3.8D), $22.14 \% \pm 1.43 \%$ colocalize with AMPAR (Figure 3.8E), and $20.04 \% \pm 1.47 \%$ are present at NMDAR containing structures (Figure 3.8F). These results, together with the PSD95 staining (Figure 3.4A), indicate that NL4 in the barrel cortex is present at a specific but as yet unidentified excitatory subtype of synapses, based on colocalization with PSD95 and VGluT1. Unfortunately, identification of the receptors that colocalize with NL4 in this brain region was not possible. 
A
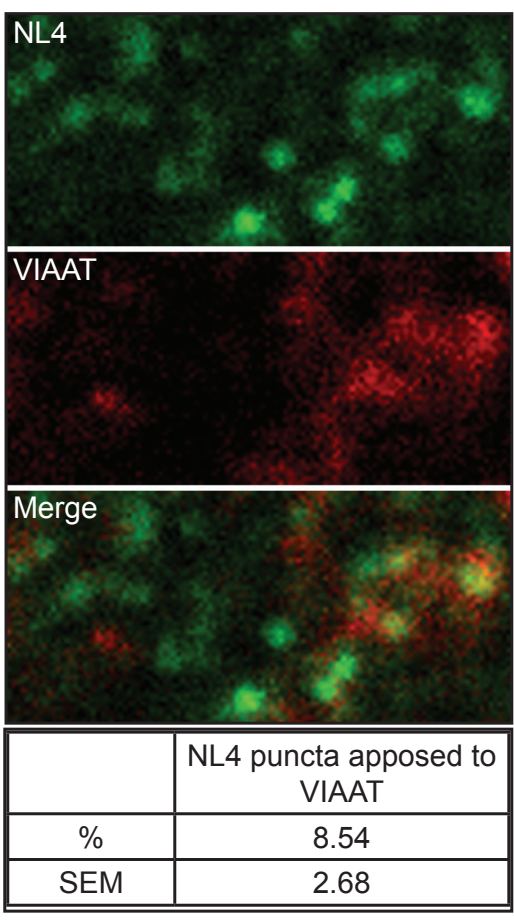

D

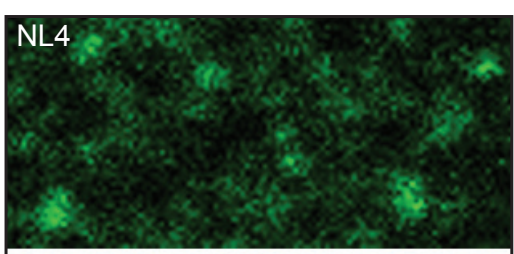

$\mathrm{GABA}_{\mathrm{A}} \mathrm{R} \gamma 2$
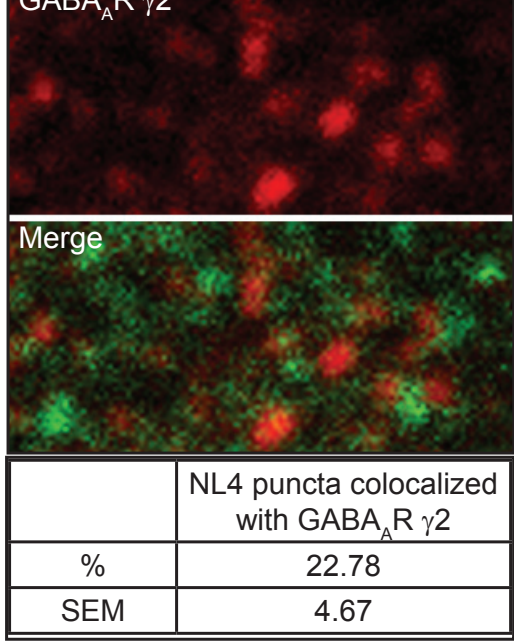

B
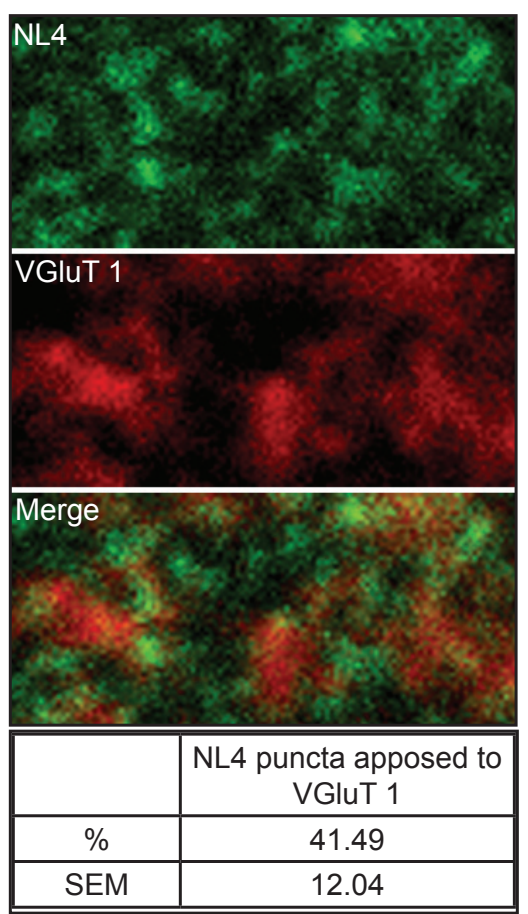

E
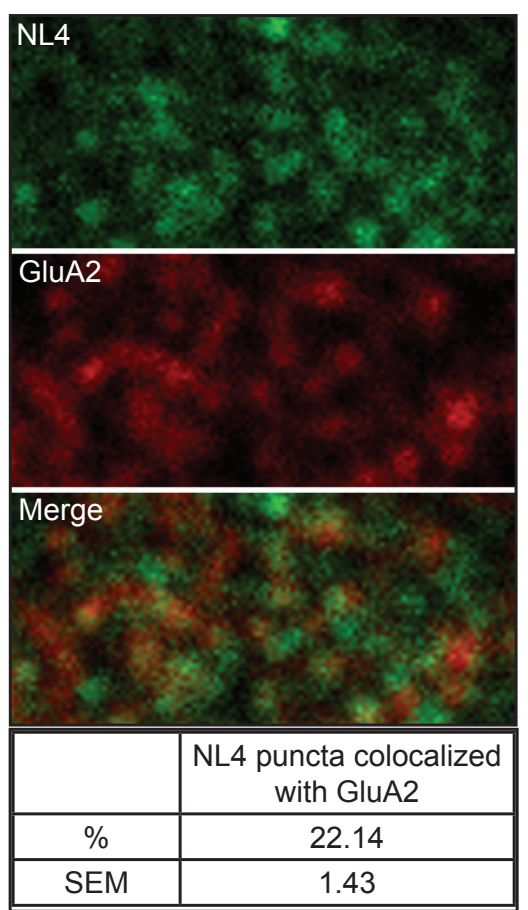

C
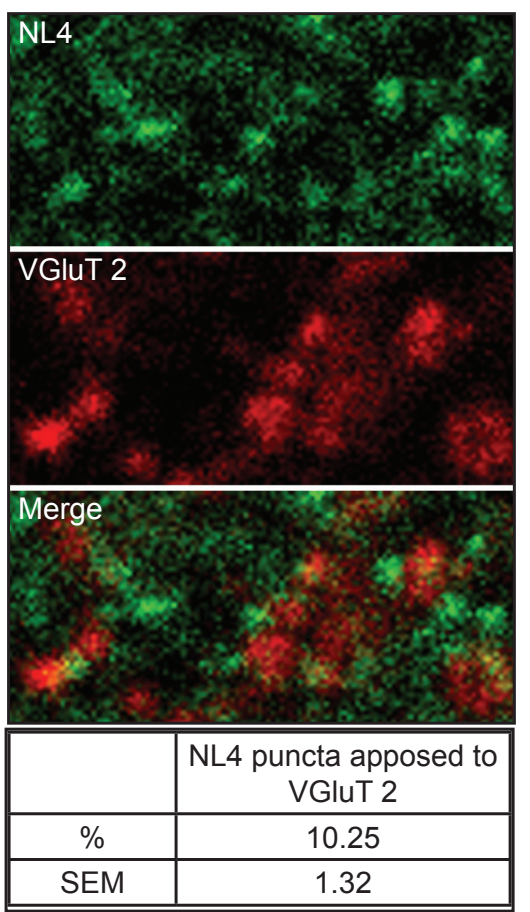

$\mathbf{F}$
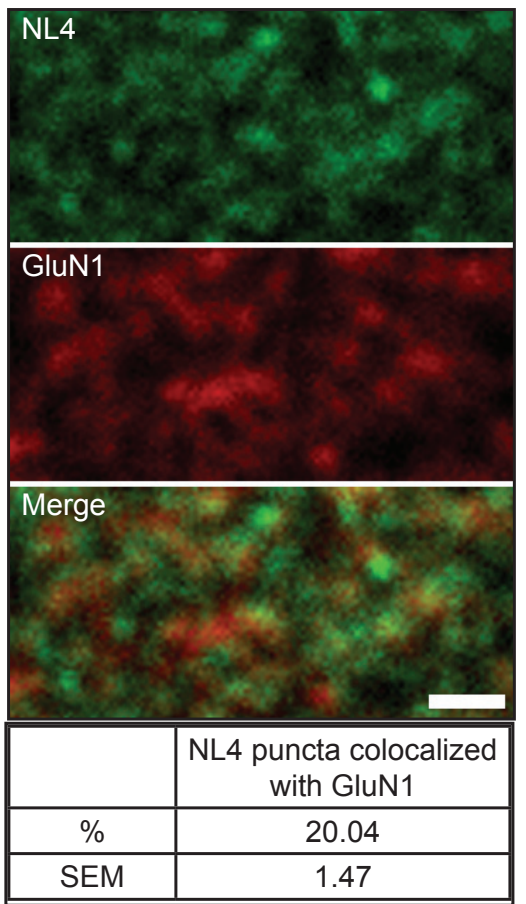

Figure 3.8: Localization of NL4 in the barrel cortex. To further characterize the distribution of NL4 in the barrel cortex, immunolabelings were carried out for NL4 together with inhibitory (VIAAT) (A) and excitatory (B, C) (VGluT1 and 2) presynapse markers, as well as with inhibitory $\left(\mathrm{GABA}_{A} \mathrm{R} \gamma 2\right.$ for the $\left.\mathrm{GABA}_{\mathrm{A}} \mathrm{R}\right)$ (D) and excitatory (GluA2 for AMPAR and GluN1 for the NMDAR) (E, F) postsynapse markers. The tables below the figures show quantification of the degree of colocalization, obtained by evaluating NL4 clusters (set by a threshold) and their colocalization with the different synaptic markers tested (set by a threshold) ( $\mathrm{n}=3$ pairs). In the barrel cortex, NL4 is mainly apposed to VGluT1 as compared to the others presynaptic markers, indicating the presence of NL4 at excitatory 
synapses. None of the labelings for postsynaptic colocalization showed NL4 to be colocalized in this study.

Scale bars: overview $=10 \mu \mathrm{m}$; detail $=1 \mu \mathrm{m}$

\subsubsection{NL4 is present at excitatory and inhibitory synapses in the hippocampus}

NL4 staining was detected in the hippocampus CA1 str. l-m (Figure 3.3C). The labeling was punctate and dense in this layer (Figure 3.9). Double immunolabeling experiments in the hippocampus revealed that NL4 is mainly apposed to VGluT1 $(33.34 \% \pm$ $9 \%$ ) (Figure 3.9B) and VIAAT $(25.35 \% \pm 2.51 \%)$ (Figure 3.9A), as compared to VGluT2 $(4.94 \% \pm 1.68 \%)$ (Figure 3.9C). At the postsynaptic side, NL4 is colocalized to a greater extent with the $\mathrm{GABA}_{\mathrm{A}} \mathrm{R} \gamma 2$ subunit present in $\mathrm{GABA}_{\mathrm{A}} \mathrm{R}(42.23 \% \pm 14.06 \%)$ (Figure 3.9) than with NMDAR $(16.19 \% \pm 1.29 \%)$ (Figure 3.9F) and AMPAR $(4.22 \% \pm 1.9 \%)$ (Figure 3.9E). Together with the PSD-95 and gephyrin labelings, showing that NL4 colocalizes with both markers (Figure 3.4B), these data indicate that in the hippocampus CA1 str. l-m NL4 could is present at both excitatory and inhibitory synapses. As in the barrel cortex, the receptors that equip NL4 excitatory synapses in hippocampus remain to be found. 
A
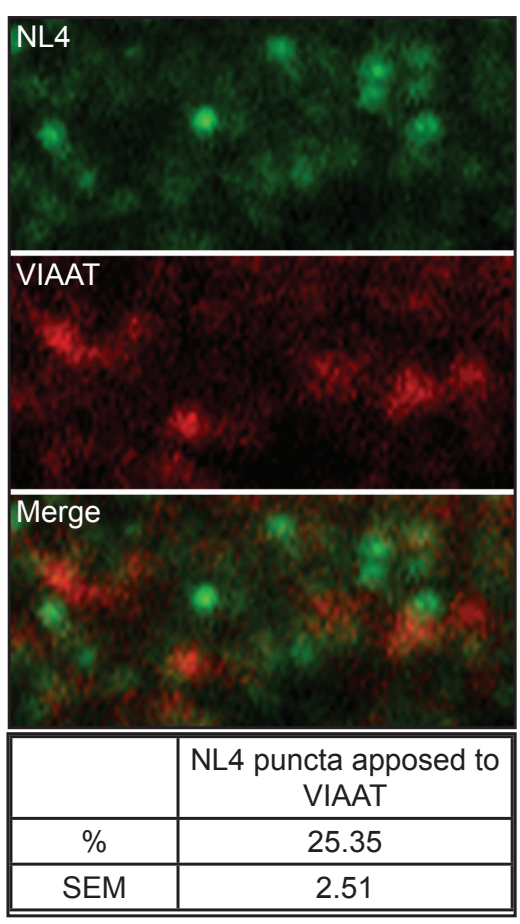

D
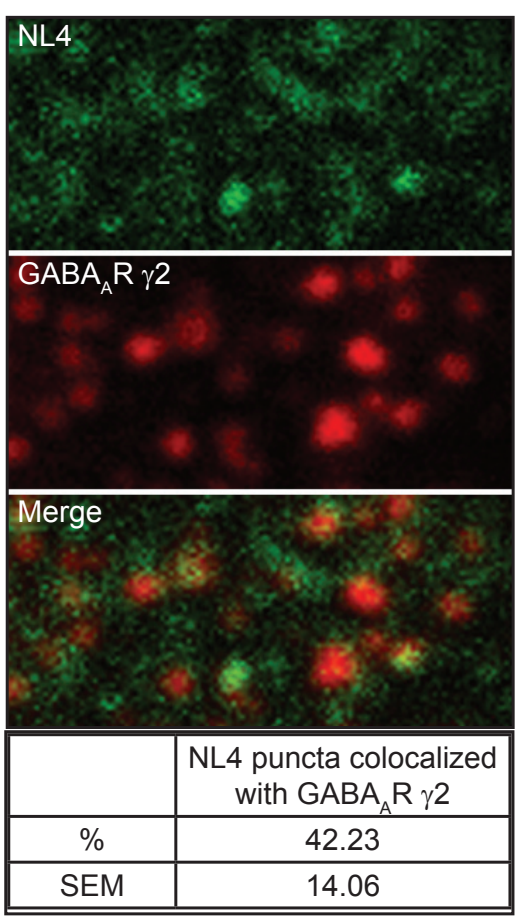

B
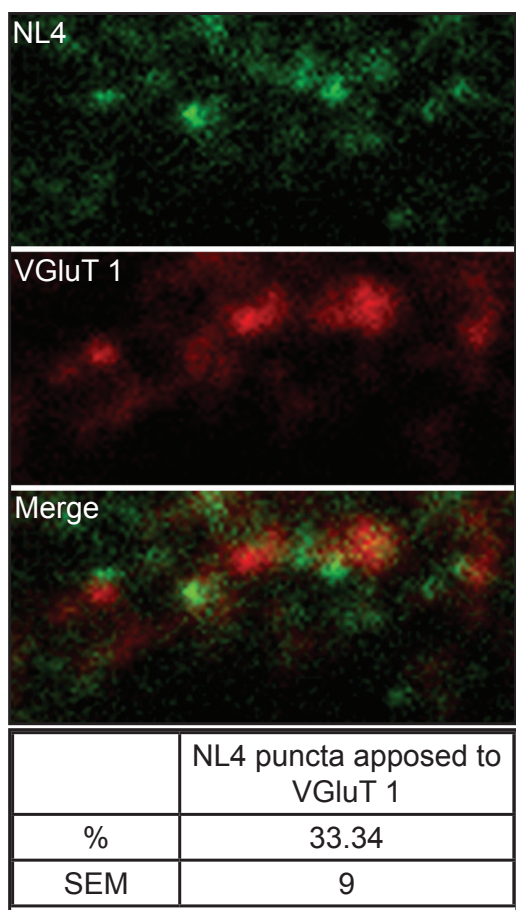

$E$
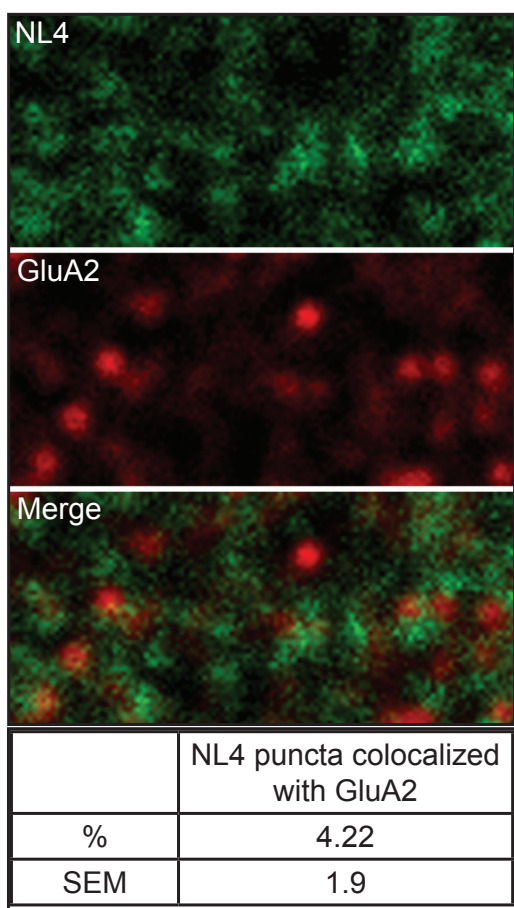

C
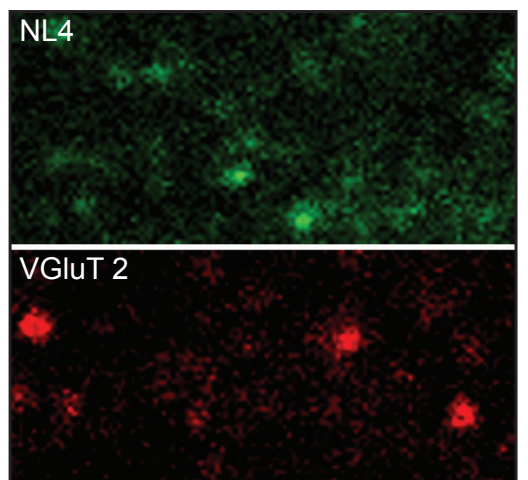

Merge

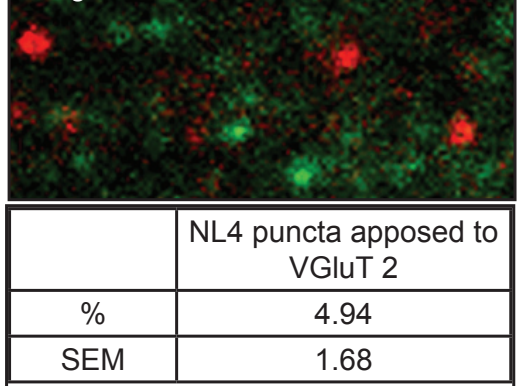

$\mathbf{F}$
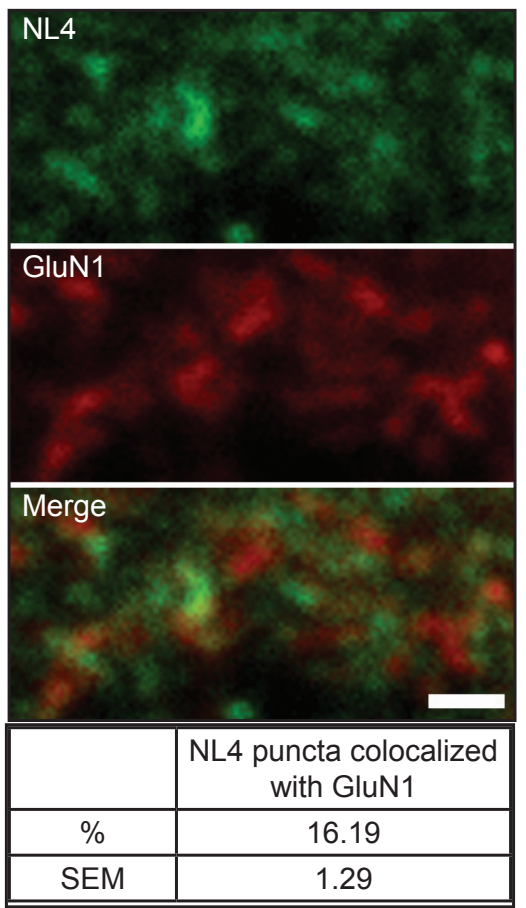

Figure 3.9: Localization of NL4 in the hippocampus (CA1 stratum lacunosum moleculare (str. l-m)). To further characterize the distribution of NL4 in the hippocampus, immunolabelings were carried out for NL4 and inhibitory (VIAAT) (A) and excitatory (B, C) (VGluT1 and 2) presynapse markers, and for inhibitory $\left(\mathrm{GABA}_{\mathrm{A}} \mathrm{R} \gamma 2\right.$ for the $\left.\mathrm{GABA}_{\mathrm{A}} \mathrm{R}\right)$ (D) and excitatory (GluA2 for AMPAR and GluN1 for the NMDAR) (E, F) postsynapse markers. Tables below figures show quantification of the degree of colocalization; obtained by evaluating NL4 clusters (set by a threshold) and their colocalization with the different synaptic markers tested (set by a threshold) ( $n=3$ pairs). In the hippocampus, NL4 is mainly apposed to VGluT1 as compared to the other presynaptic markers, indicating the presence of 
NL4 at excitatory synapses. At the postsynaptic side, I found that approximately half of the NL4 puncta colocalize with the GABA ${ }_{A} R \gamma 2$ subunit of $G_{A B A}$ receptors. Both quantification results indicate that NL4 in the hippocampus is present at excitatory and inhibitory synapses. Scale bars: overview $=10 \mu \mathrm{m}$; detail $=1 \mu \mathrm{m}$

\subsection{Characterization of NL4 deletion mutant mice}

\subsubsection{NL4 knockout mice show no alteration in synaptic circuitry}

To examine if the deletion of NL4 in mice results in aberrant synaptogenesis, a detailed analysis of synapse densities was performed in two regions expressing NL4, the primary somatosensory cortex layer IV (barrel cortex) and CA1 str. l-m. NL4. Synaptic cell adhesion proteins are thought to be involved in initial synaptogenesis and in the maturation of synapses. If NL4 were involved in initial synaptogenesis, its deletion would lead to a change in synapse density in specific brain regions. To quantify synapse density, immunolabelings utilizing postsynaptic markers were carried out in pairs of WT and NL4-KO mice (Figure 3.10). Specifically, immunostaining of barrel cortex and CA1 str. 1-m for the postsynaptic markers gephyrin and PSD-95 was carried out (Figure 3.10A). No differences were observed in the density of PSD-95 (54 puncta/100 $\mathrm{m}^{2}$ for WT and 58 puncta/100 $\mu \mathrm{m}^{2}$ for the NL4-KO) and gephyrin puncta (44 puncta/ $100 \mu \mathrm{m}^{2}$ for the WT and 46 puncta/100 $\mu \mathrm{m}^{2}$ for the NL4-KO) in the barrel cortex (Figure 3.10B). Similarly, in the hippocampus, no significant difference was observed in PSD-95 (92 puncta/100 $\mu^{2}$ for the WT and 87 puncta/100 $\mu \mathrm{m}^{2}$ for the NL4KO) or in gephyrin puncta (63 puncta/ $100 \mu \mathrm{m}^{2}$ for the WT and 77 puncta/100 $\mu \mathrm{m}^{2}$ for the NL4KO) (Figure 3.10B). Both synaptic markers were present at equal quantities in the NL4-KO and WT mice (Figure 3.10B). Therefore, the loss of NL4 does not appear to affect synaptogenesis in the barrel cortex or in the hippocampus.

The ratio of excitatory to inhibitory synapses is crucial for neuronal network function. In the barrel cortex, the ratio of PSD95 puncta to gephyrin puncta was $1.23 \pm 0.13$ in the WT and $1.28 \pm 0.2$ in the NL4-KO animals (Figure 3.10D). In the hippocampus the corresponding ratio was $1.53 \pm 0.2$ in the WT and $1.23 \pm 0.17$ in the NL4-KO mouse (Figure 3.10D). Even though a trend towards lower ratios was found in the hippocampus, no significant differences were observed. It is possible, though, that with an increased sample number a significant difference might be obtained. 
A
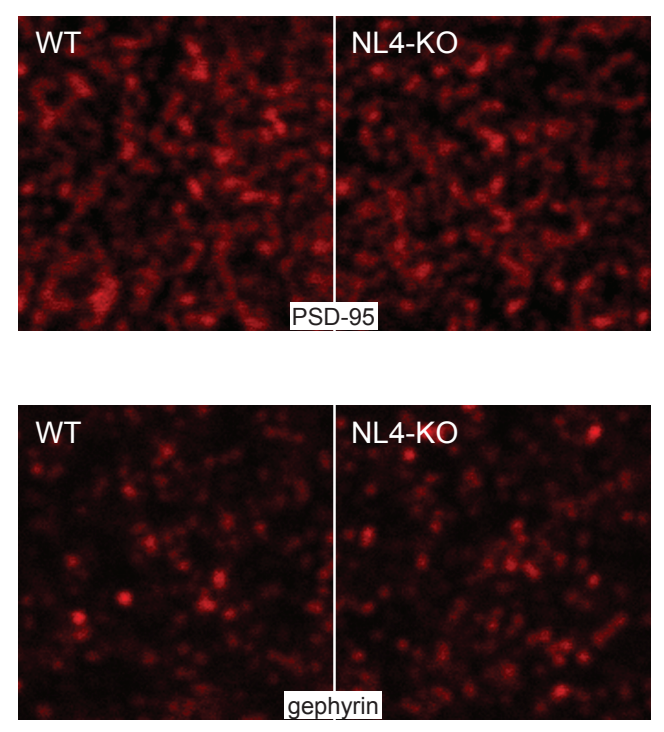

C

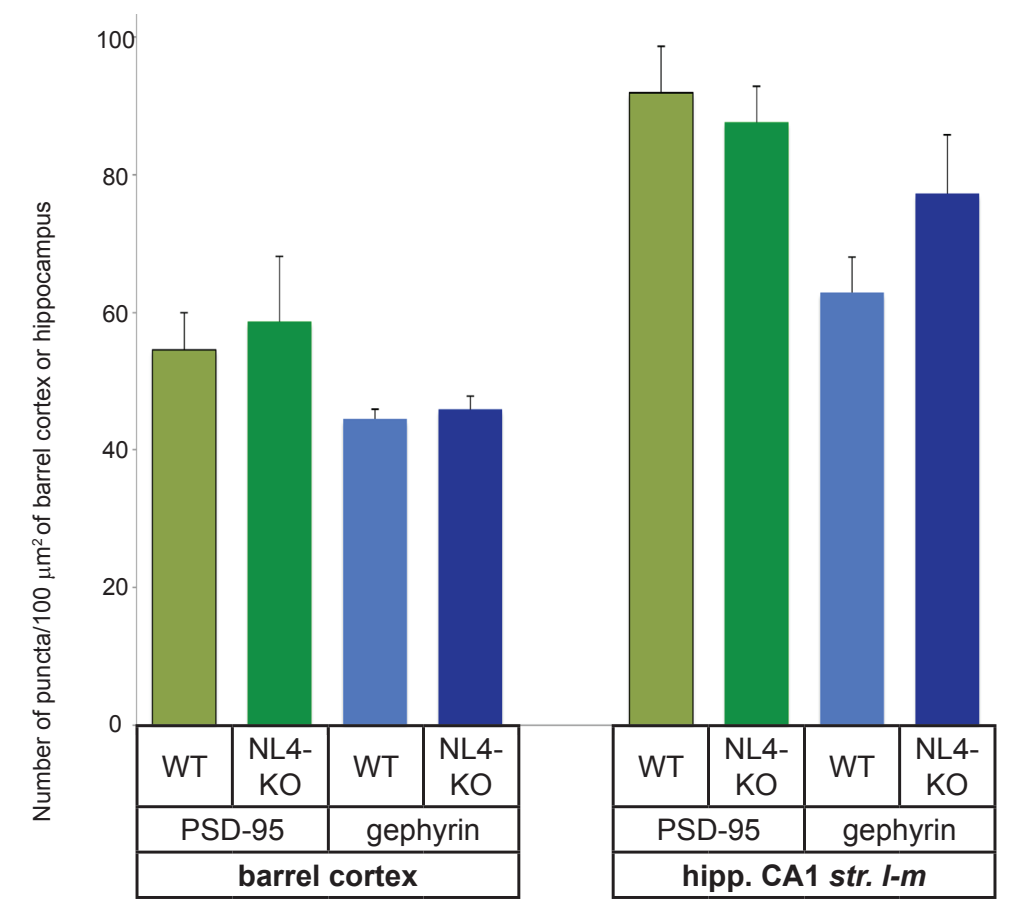

B
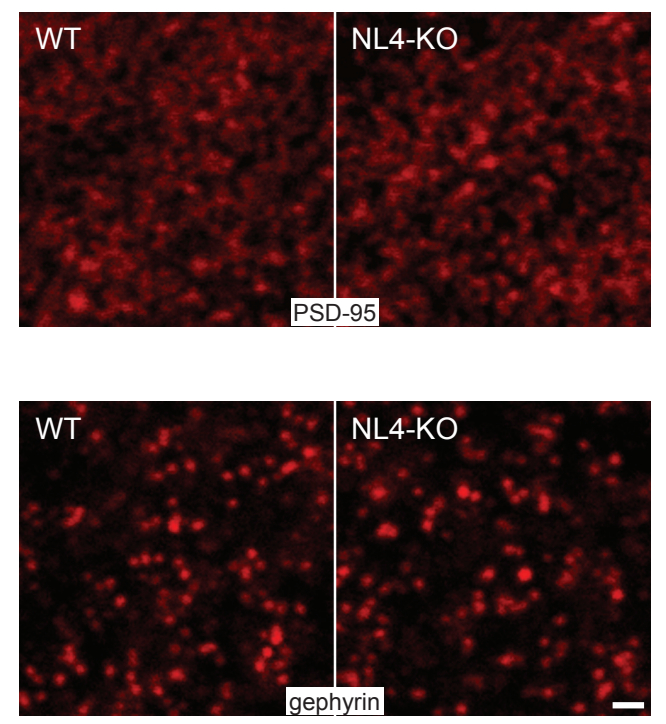

D

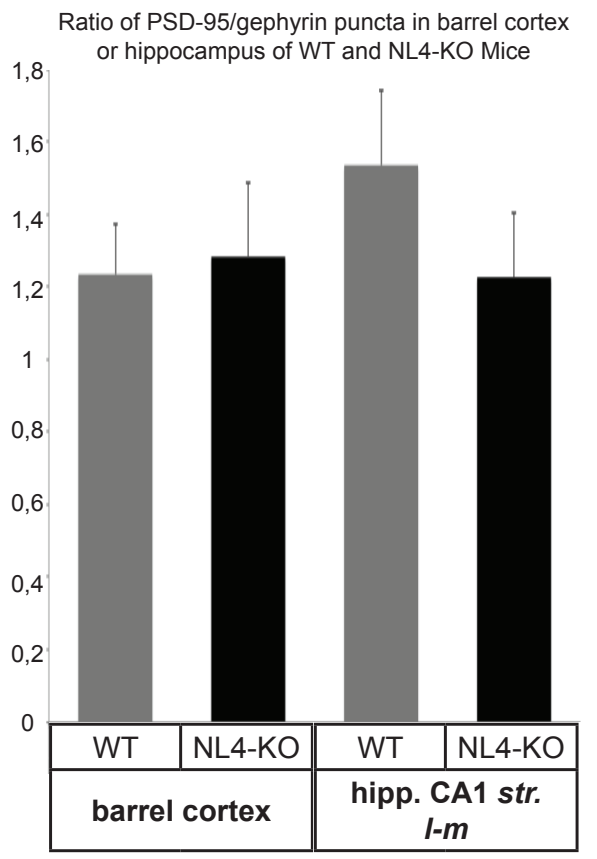

Figure 3.10: No alterations in excitatory and inhibitory synapse density in the NL4-KO barrel cortex or hippocampus. Confocal images showing PSD-95 and gephyrin immunoreactivity in the barrel cortex and CA1 stratum lacunosum moleculare of hippocampal coronal sections. (A, B) Immunostaining of PSD-95 for excitatory synapses and gephyrin for inhibitory synapses was used to quantify the synapse density in barrel cortex (A) and hippocampus (B) of WT and NL4-KO mice. (C) No significant changes were observed in the density of PSD-95 or gephyrin puncta in the barrel cortex and hippocampus of NL4 knockout mice, or (D) in the ratio of PSD-95 puncta versus gephyrin puncta in these brains regions ( $n=6$ pairs) Error bars represent SEM.

Scale Bar $=1 \mathrm{~mm}$ 
In the retina, a small decrease of GlyR $\alpha 1$ positive puncta was observed in the NL4KO mice (Hoon et al., 2011). Here, the same analysis was carried out in the spinal cord to investigate the consequences of NL4 deletion because NL4 is strongly immunoreactive in the spinal cord (Figure 3.1A). Immunolabelings for the GlyR $\alpha 1$ subunit did not show any major changes in the staining between WT and NL4-KO (Figure 3.11A). Furthermore, no changes were observed in the glycinergic synapse density between the WT (15 puncta/100 $\mu^{2}$ ) and the NL4-KO (16 puncta/100 $\mu^{2}$ ) mice (Figure 3.11B).

A

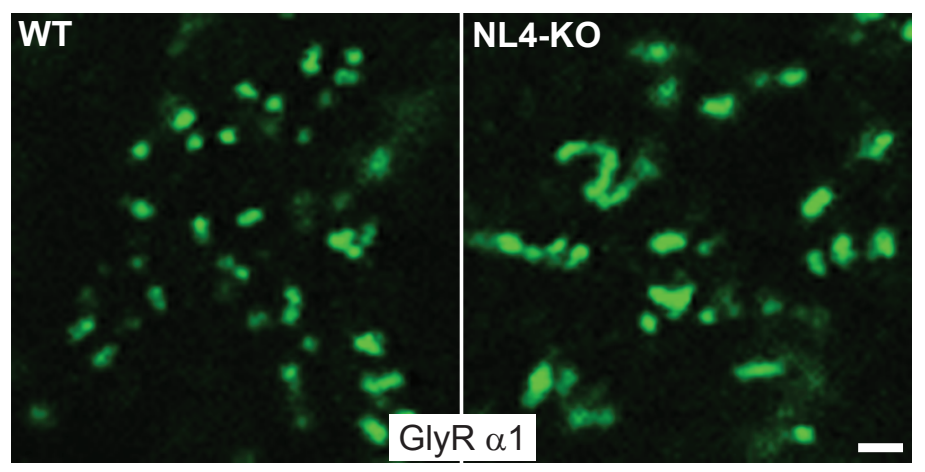

B

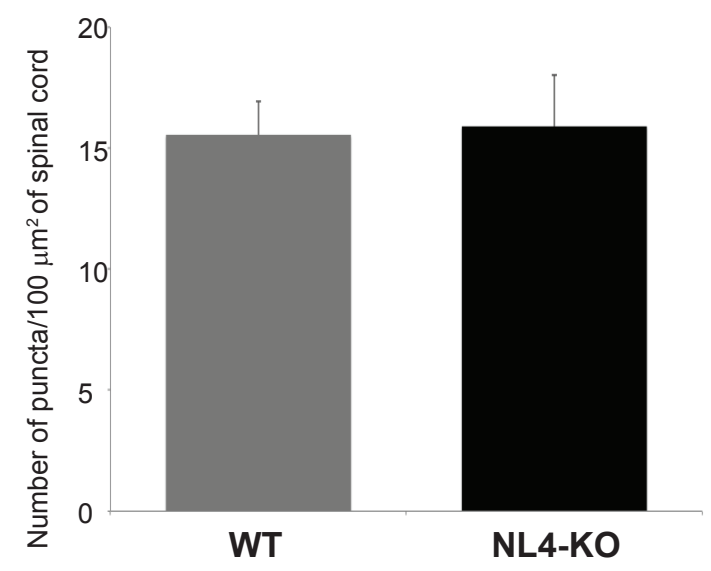

Figure 3.11: No alterations in glycinergic synapse density in the NL4-KO spinal cord. (A) Representative labeling of a subset of GlyR synapses (GlyR $\alpha 1)$ used to compare the synapse density in WT and NL4-KO mice in the dorsal horn of the spinal cord. (B) No significant changes were observed in the glycinergic synapse number in the spinal cord of NL4 knockout mice (n = 5 pairs). Error bars represent SEM. Scale Bar $=1 \mathrm{~mm}$ 


\subsubsection{Upregulation of neuroligins isoforms as a consequence of the NL4 knockout}

To test if the loss of NL4 in KO mice is compensated by an upregulation of the expression of other NLs, I performed a quantitative immunoblot using NL isoform-specific antibodies and tissue from adult WT and NL4-KO mice (illustrated Figure 3.12A). The levels of NL1-3 protein expression were the same in globus pallidus and brainstem of NL4-KO and control mice. However, in the hippocampus, a trend towards an upregulation of NL2 was observed $(114.8 \% \pm 7.6 \%$ in the NL4-KO, normalized to the WT levels) (Figure 3.12B). In the barrel cortex, a small but significant upregulation of NL2 $(117.6 \% \pm 3.6 \%)$ and NL3 $(119.6 \% \pm 6.6 \%)$ was observed in NL4-KO compared to the control levels $(\mathrm{p}<0.05$, paired Student's test) (Figure 3.12B).

A

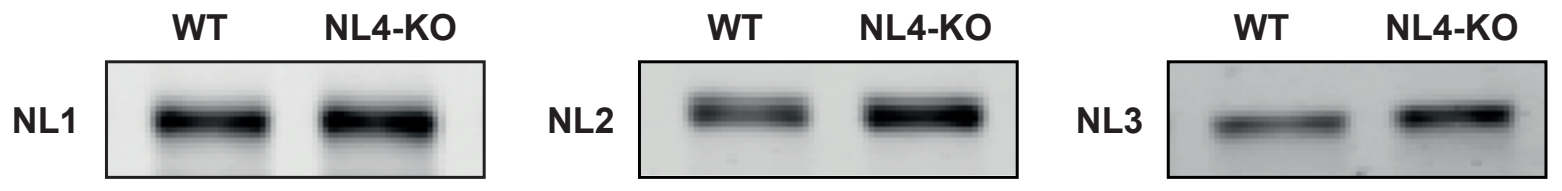

B

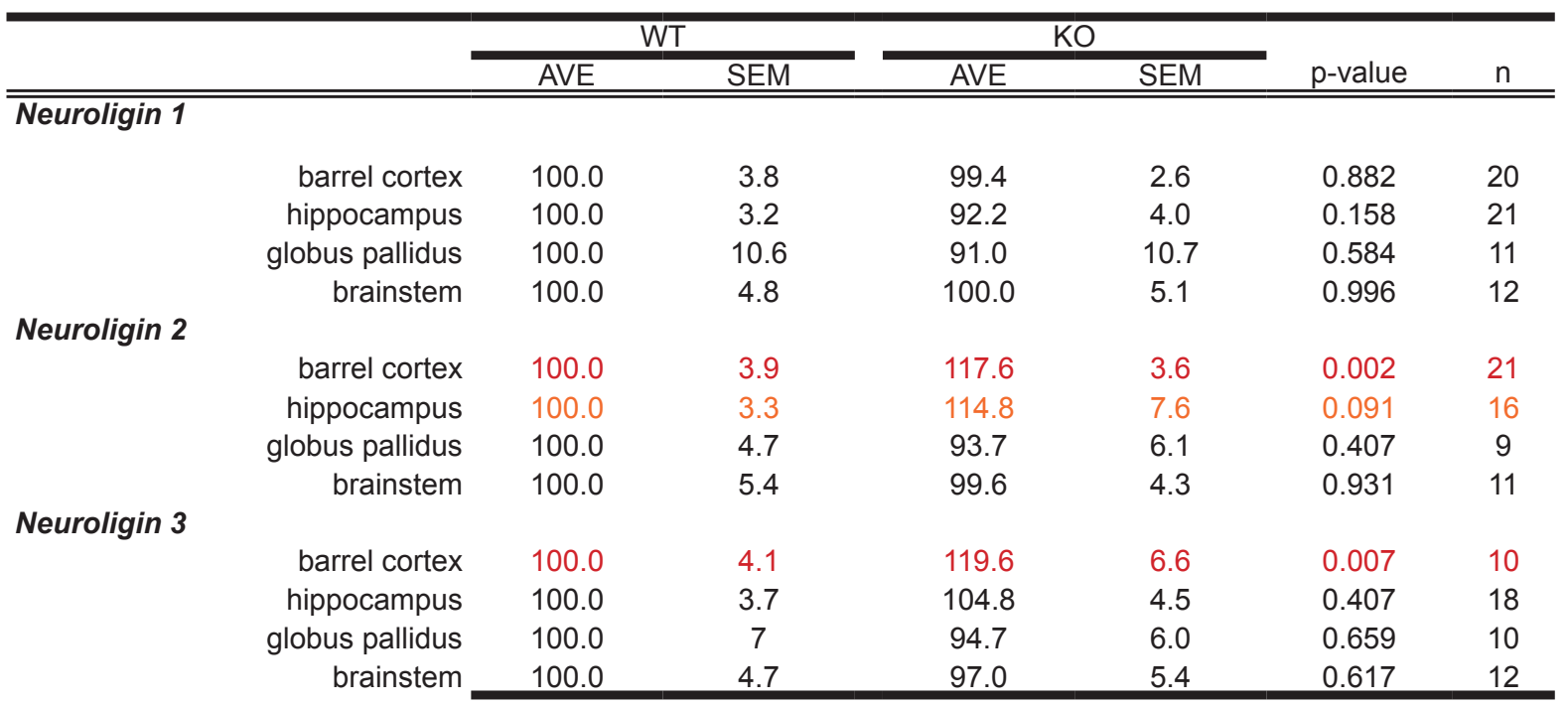

Figure 3.12: Upregulation of NL2 and NL3 in the absence of NL4. Quantitative immunoblot analysis of neuroligin isoforms 1-3 in tissue from adult WT and NL4-KO mice. (A) Sample immunoblotting of NL1, NL2 and NL3 in barrel cortex of WT and NL4-KO mice. (B) Quantification of NL1-3 in different brain regions of WT and NL4-KO mice, normalized to WT levels. Significant differences ( $\mathrm{p}<0.05$, paired Student's t-test) are highlighted in red; trends ( $\mathrm{p}<0.1$, paired Student's t-test) are highlighted in orange. ' $\mathrm{n}$ ' refers to the number of WT/NL4-KO pairs analyzed. 


\subsubsection{NL4-KO mice have strongly reduced gamma oscillations in CA3}

The above results indicate that NL4 might not play a role in initial synaptogenesis. Another role that has been proposed for NLs is in the maturation of synapses (Varoqueaux et al., 2006). According to this model, NL4 might influence the composition of synapses by affecting receptor clustering (Hoon et al., 2011), and NL4 deletion might therefore disturb synapse function and network activity, e.g. in the hippocampus and the barrel cortex. Based on the fact that NL4-KOs show no signs of aberrant synapse formation or of alterations in glutamatergic or GABAergic postsynapses in the brain (Figure 3.10). I expected that effects of NL4 deletion at the level of synaptic function would be difficult to reliably assess electrophysiologically. Consequently, electrophysiological analysis was focused on network function in hippocampus. In this brain region, global neuronal activity can be recorded by inducing oscillations such as the ones belonging to the gamma range. Gamma oscillations in the hippocampus can be induced by the application of kainate. In the present study, the generation and maintenance of kainate-induced gamma oscillations was analyzed in NL4-KO animals and WT littermate controls.

Oscillation measurements were done under interface conditions, in which the slice was kept at the interface below a flow of artificial cerebral spinal fluid (ACSF). Oscillations in the gamma frequency range were induced pharmacologically by kainate application in the ACSF solution (100 $\mathrm{nM}$ kainate). Under these conditions, oscillations were induced within $10 \mathrm{~min}$ after drug application. Long-lasting and stable rhythmic activity was induced in the hippocampal slices and recorded in CA3 (Figure 3.13A) and CA1. Gamma oscillations induced in CA1 region in the hippocampus are known to be weaker than the ones present in CA3, and under the conditions used in this study, the amplitude of oscillations in CA1 was too low to allow for reliable quantification after baseline subtraction. Therefore, only data for oscillations in area CA3 are shown.

Oscillatory activity in CA3 could be induced with $100 \mathrm{nM}$ kainate with an amplitude of around $200 \mu \mathrm{V}$. Application of $100 \mathrm{nM}$ kainate induced gamma oscillations with significantly smaller power in the NL4-KO mice compared to the control (Figure 3.13B). The power of the maximum peak was $43.93 \pm 12.9 \mu \mathrm{V}^{2} / \mathrm{Hz}$ for the NL4-KO mice and $119.75 \pm$ $8,36 \mu \mathrm{V}^{2} / \mathrm{Hz}$ for the control ( $\mathrm{n}=6$ pairs, 3 slices per animal, $\mathrm{p}<0.05$, paired Student's test) (Figure 3.12C). Furthermore, the frequency of the maximum peak in the power spectrum was not significantly different in the NL4-KO mice as compared to the control $(33.56 \pm 1.44 \mathrm{~Hz}$ in 
NL4-KO, $33.79 \pm 1.14 \mathrm{~Hz}$ for WT) (Figure 3.13D). These data indicate that the neuronal network involved in hippocampal gamma oscillation is impaired in NL4-KO mice.

A

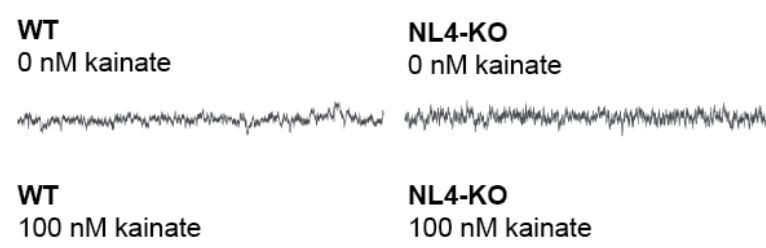

C

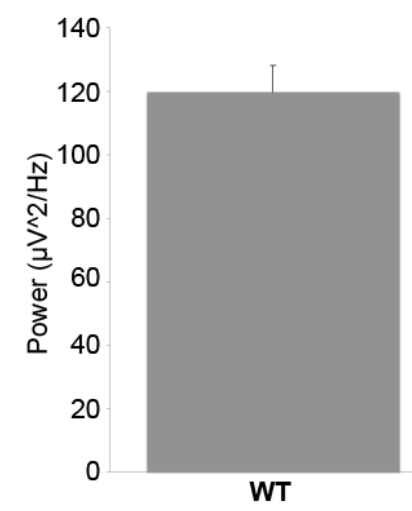

B

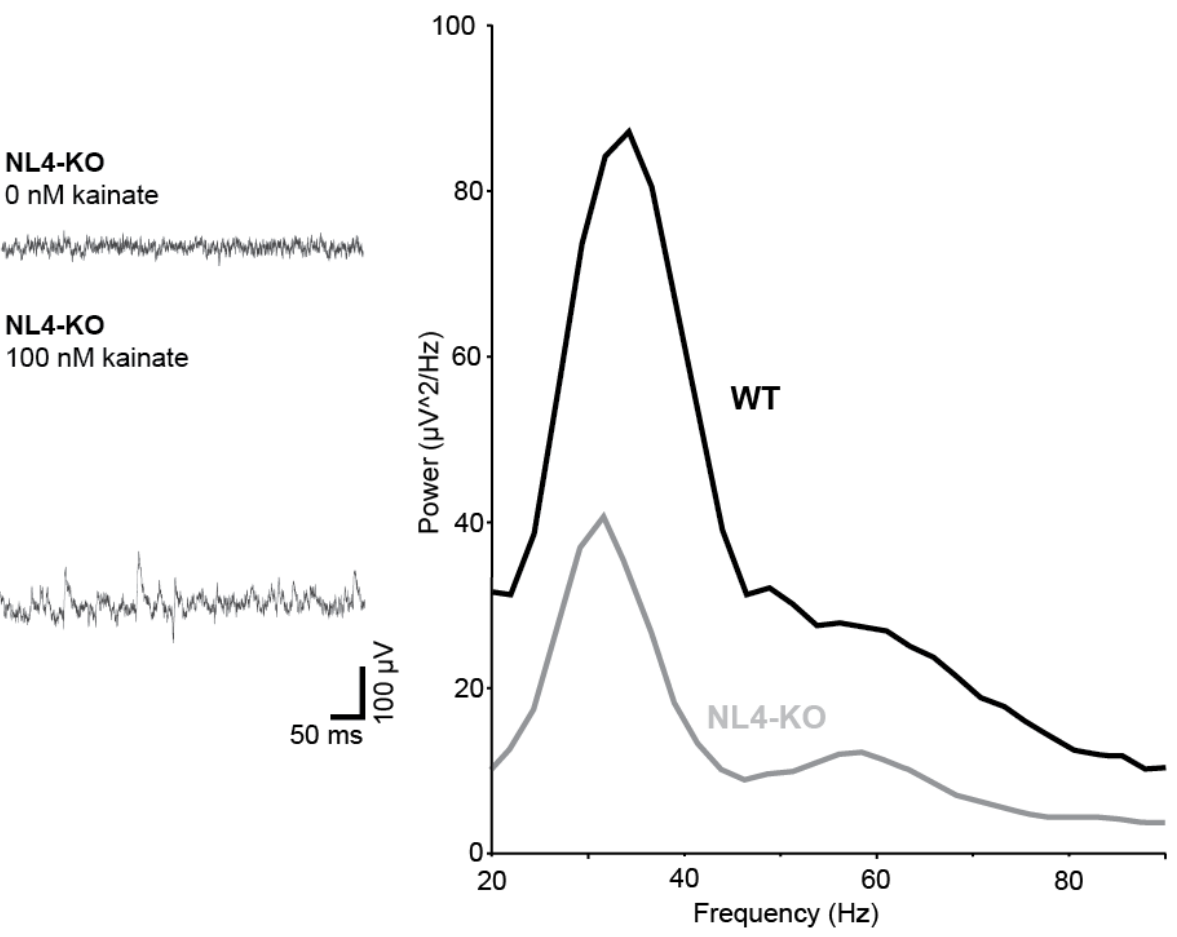

D

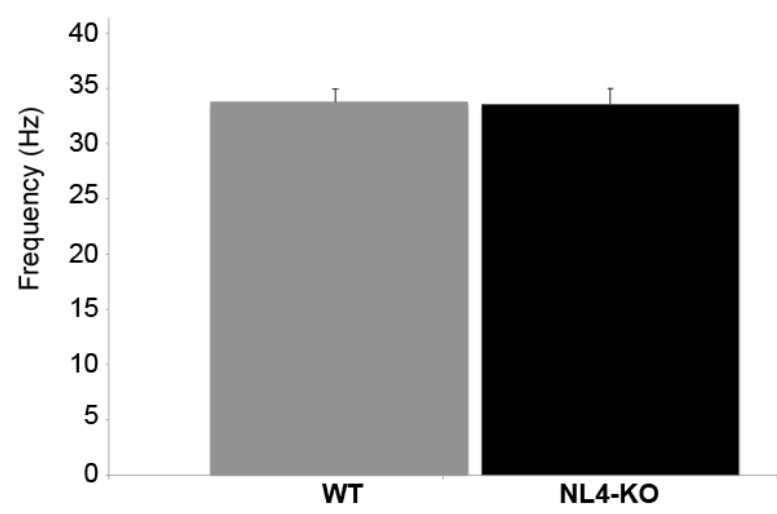

Figure 3.13: Reduced hippocampal gamma oscillations in NL4 knockout mice. (A) Gamma oscillations were induced by the application of $100 \mathrm{nM}$ kainate under interface conditions. (B) The power of the oscillations in NL4-KO hippocampal slices was reduced as by the power spectrum from wild type (WT) (grey) and NL4 mutant mice (black). The power spectrum shows the reduced gamma oscillation power in the NL4 KO as recorded from the CA3b region of the hippocampus in the presence of $100 \mathrm{nM}$ kainate. (C) Quantification of the power of the maximum peak revealed a significant decrease in power in mutant slices (black) as compared to control (grey) animals. Significant differences ( $p<0.05$, paired Student's t- 
test) are marked with an asterisk. (D) The mean frequency of the maximum power peak was not changed in the NL4-KO slices as compared to control slices ( $n=6$ pairs, average of 3 slices per animal). Error bars represent SEM. 


\section{Discussion}

NLs are ubiquitous postsynaptic adhesion proteins that are believed to be essential for synapse maturation and function. Mutations of NL4 have been associated with autism-related disorders in humans. Furthermore, NL4-KO mice were found to show specific behavioral phenotypes that mimic those observed in autism in humans (Jamain et al., 2008). In the present study, the distribution of NL4 was characterized in the mouse CNS. The highest NL4 protein expression levels were observed in brainstem and spinal cord (Figure 3.1). Detailed analysis demonstrated that NL4 is localized to a majority of glycinergic postsynapses in the brainstem and spinal cord (Figure 3.7) (Hoon et al., 2011). Moreover, NL4 was found to be localized at inhibitory, GABAergic synapses in the globus pallidus (Figure 3.5). Interestingly, NL4 is not exclusively localized to inhibitory synapses. Instead, its synaptic localization and corresponding interaction partners appear to be dependent on the brain region. Specifically, NL4 was also found at excitatory synapses in the barrel cortex (Figure 3.8), and in the hippocampus NL4 is localized to both inhibitory and excitatory synapse types (Figure 3.9).

To investigate the role of NL4 at synapses, the consequences of NL4 deletion on synaptogenesis were studied. In the barrel cortex and hippocampus, the loss of NL4 did not influence the density of excitatory or inhibitory synapses (Figure 3.10). Although it was shown previously that the density of glycinergic receptors slightly decreases in the retina of NL4-KO mice (Hoon et al., 2011), no such change was found in the spinal cord (Figure 3.11). Since no alterations in synaptic density were observed in any of the screened brain regions, it is likely that other NL isoforms or cell adhesion proteins may compensate for the deletion of NL4. Accordingly, a localized compensatory change in the expression of NL2 and NL3 in the barrel cortex of NL4-KO mouse was found (Figure 3.12). Since the lack of NL4 did not appear to have an effect on the total number of synapses, the next step of investigation was to look at the maturation and functionality of the existing synapses. To this end, gamma oscillations were induced in the hippocampus. Interestingly, in the absence of NL4, the power of gamma oscillations in the hippocampus was greatly reduced (Figure 3.13). Together, these data provide new important insights into the physiological consequences of NL4 loss in autism spectrum disorders. 


\subsection{Characterization of NL4 distribution by immunohistochemistry}

The determination of the NL4 immunoreactivity pattern is a key step towards the characterization of NL4 function and a prerequisite for the identification of the brain regions involved in the ASD-related functions of NL4. In order to achieve this, morphological analyzes were performed to examine the distribution pattern of NL4 throughout the brain and spinal cord (Figure 3.1) using immunohistochemistry.

\subsubsection{Methodological considerations for NL4 immunohistochemistry}

Studies based on immunohistochemistry are critically dependent on the quality of antibodies available and on the exact immunostaining protocols used. Prior to this study, no protocols had been established for the use of NL4 antibodies in immunostaining procedures. It was therefore necessary to first characterize and validate the methodology applied here. The antibody used was a polyclonal antibody directed against the extracellular domain of NL4. Like many antibodies against postsynaptic proteins (Schneider Gasser et al., 2006), this antibody did not work with conventionally perfused tissue sections. Instead, a fast brain extraction without perfusion was used, followed by weaker fixation using either methanol or PFA and subsequent antigen retrieval. The validity of these labelings was established by the absence of a specific signal in NL4 mutant mice (Figure 3.1). For all subsequent experiments, a NL4 knock out control was used in parallel to the WT tissue to ensure specificity. Image processing parameters were set such that no signal was visible in the NL4-KO section, and the same settings were applied to WT brain sections (including signal amplification value for confocal image acquisition and threshold for the quantification) (Figure 3.1). This stringent approach ensured that specific NL4 staining could be reliably identified even in those brain regions where the signal was relatively faint and diffuse [such as in some areas as barrel cortex and hippocampus (Figure 3.4A-B)].

One complication arising from the fixation conditions required to obtain reliable NL4 staining was that these conditions were not optimal for co-labeling with certain other markers, such as VIAAT and VGluT. The fixation required for the optimal NL4 immunostaining, i.e. methanol fixation, was not adequate for labeling with presynaptic markers and some postsynaptic markers. Compromises were found that permitted the observation of NL4 with a presynaptic marker. However, these compromises inevitably resulted in some sacrifices 
regarding the quality of the immunostaining. Quantification of data from the colocalization experiments in some cases resulted in a small percentage of artificially or non-specifically apposed or colocalized puncta due to these experimental limitations. Nevertheless, results obtained from these experiments provided important data on the colocalization or apposition of presynaptic markers with NL4 as discussed below.

\subsubsection{Distribution of neuroligin 4 in regions of the central nervous system that are relevant for ASDs}

In autistic individuals, anatomical abnormalities have been reported in several brain areas, such as reduced numbers of Purkinje cells in the cerebellum and increased cell packing density in the limbic system (Bauman and Kemper, 2005). Correlating NL4 protein immunoreactivity with equivalent regions in the mouse brain would be an important first step in elucidating the role of NL4 in ASDs.

One of the most prominent areas that display clear NL4 labeling is layer IV of the cortex (Figure 3.2B-D), with an intense staining observed in the barrel field of the primary somatosensory area. The barrel cortex in rodents is the region of the somatosensory cortex that processes sensory information arriving from the whiskers. Whisking allows rodents to screen their habitat and to perform complex orientation and discrimination tasks (Brecht et al., 1997; Diamond et al., 2008), and tactile information from the whiskers is relayed through defined pathways to the primary somatosensory cortex. The neurons of layer IV are arranged in functional units called barrels, each of which processes tactile input principally from a single whisker. This sensory pathway offers unique opportunities for linking the architecture of specific synaptic microcircuits to cortical sensory processing, and it may provide the opportunity to study the function of NL4 in a particular microcircuit.

The observation that NL4 is immunoreactive highly in somatosensory cortex is interesting, since individuals with autism are known to have alterations in sensory processing. Using psychophysical ratings and functional magnetic resonance imaging (fMRI) to investigate responses to somatosensory stimulation in humans, it was shown that patients with ASD have diminished responses to pleasant and neutral stimuli and exaggerated responses to unpleasant stimuli (Cascio et al., 2012). Therefore, the barrel cortex in NL4-KO mice may be 
a useful model system for studying correlations between alterations in synaptic architecture and functionality and disturbances in somatosensory processing in future studies.

Another region that showed prominent NL4 labeling was the hippocampus. Interestingly, several post-mortem studies on autistic brains have emphasized the hippocampus as being one of the seats of morphological changes in ASD (Bauman and Kemper, 2003; Schumann et al., 2004; Halpain et al., 2005). Moreover, mice with an autismrelated NL3 knock-in $\left[\mathrm{NL} 3 \mathrm{R}^{451} \mathrm{C}\right]$ mutation show a distinct phenotype in the CA1 region of the hippocampus, including an increased dendritic extension, altered structure of synapses in the str. rad, increased AMPAR-mediated excitatory synaptic transmission, a dramatic alteration of NMDAR -mediated synaptic response kinetics and a twofold up-regulation of NMDARs containing GluN2B subunits (Etherton et al., 2011). These results indicate that specific circuitry impairments in the hippocampus, and specifically those due to mutation of NLs, may be involved in the pathogenesis of ASDs.

Strikingly, NL4 is specifically localized in the CA1 str. $l-m$, and is much less abundant in the rest of the hippocampus (Figure 3.2D). A previous study showed strong NL4 gene expression in the pyramidal cell layer of hippocampus (Jamain et al., 2008). Together these data emphasize a role of NL4 at the distal dendrites of pyramidal cells in the hippocampus.

CA1 pyramidal cells receive two different inputs in the str. 1-m. One of these is the excitatory input from the perforant path of the entorhinal cortex. CA1 pyramidal cells activated by the perforant path will in turn activate various inhibitory interneurons in area CA1. One class of interneurons, the O-LM cells, receive excitatory EPSPs from pyramidal cell axons and Schaffer collateral branches in the str. or (Lacaille et al., 1987; Ali and Thomson, 1998), and in turn innervate the distal dendrites of pyramidal cells in the str. $l-m$ to evoke IPSPs (Maccaferri et al., 2000). Essentially, the direct cortical input from the perforant path excites CA1 pyramidal cells (Yeckel and Berger, 1990; Colbert and Levy, 1992; Yeckel and Berger, 1995), and at the same time activates interneurons present in the str. $l-m$, which in turn project to CA1 pyramidal cells (Empson and Heinemann, 1995a; 1995b; Remondes and Schuman, 2002). Given the importance of the prominent localization of NL4 within this circuitry, it will be interesting to determine what the effect of loss of NL4 is on synapse density and function in this region, and whether these changes may play any role in the genesis of autism-related behavioral phenotypes. Indeed, previous studies showed NL2 to be localized at perisomatic 
synapses of pyramidal cells in the CA1 str. pyr, and deletion of NL2 induced a decrease in the inhibitory synaptic density and decreased synaptic transmission from fast-spiking interneurons (Gibson et al., 2009; Poulopoulos et al., 2009). Since NL2 plays a role in the perisomatic inhibition in the hippocampus but has no effect on the synapses on distal dendrites of pyramidal cells, NL4 could be a new candidate that modulates synaptogenesis or synapse maturation on distal dendrites.

A third forebrain region that showed noticeable NL4 staining was the globus pallidus (Figure 3.2B). The globus pallidus is a part of the basal ganglia, a group of nuclei that act as a cohesive unit in voluntary motor control and procedural learning, and that are affected in disorders such as obsessive compulsive disorder and Tourette syndrome. A significant number of studies have linked structural and functional abnormalities in the basal ganglia and associated corticostriatal circuits to the repetitive behaviors observed in individuals with autism. Most of these studies have focused on the caudate and putamen, rather than on the globus pallidus specifically. However, a recent study in a group of children with ASD showed that repetitive and stereotyped behavior was associated with an increased volume in several brain regions, including the right globus pallidus (with a trend for the left hemisphere) and left and right putamen (Estes et al., 2011). Therefore, an understanding of the role of NL4 at synapses in the globus pallidus may be highly relevant to the investigation of repetitive and stereotyped behaviors in the NL4 mouse model of autism.

In addition, from a basic neuroscience perspective, one of the interesting aspects of studying NL4 in the globus pallidus is its unusually high density of inhibitory synapses. Most of the pallidal neurons are very large, PV-positive GABAergic interneurons, with very large dendritic arborisations. The primary inputs to the globus pallidus are GABAergic inhibitory afferents from the putamen, and therefore the vast majority of the synapses present in the globus pallidus are GABAergic (Difiglia and Rafols, 1988). There is very little local connectivity, and the excitatory glutamatergic inputs from the subthalamic nucleus are sparse. Less than $10 \%$ of synapses onto the globus pallidus are excitatory (Falls et al., 1983; Kita and Kitai, 1987; Shink and Smith, 1995). Given that NL4 is almost exclusively localized to inhibitory synapses in the retina, spinal cord and brainstem, the globus pallidus provides the perfect system to study the role of NL4 at inhibitory synapses in the forebrain. 
Perhaps the most prominent and intense NL4 staining in the CNS was observed in the brainstem (Figure 3.2F). Among its many functions, the brainstem is a relay for auditory information that transits through the cochlear nuclei and superior olivary complex of the brainstem. Among other sensory disturbances, autistic patients often exhibit a hypersensitivity to auditory stimuli (Rubenstein and Merzenich, 2003). In part, these disturbances are thought to be due to the poor development of cortical tonotopic maps in these patients, but it is likely that other relays in the auditory pathway are also altered. For example, an investigation of neuronal morphology in the auditory brainstem of individuals with ASDs revealed that neurons in the medial superior olive were smaller and rounder than in controls (Kulesza et al., 2011). In the same study, analysis of the human superior olivary complex, an important auditory brainstem center, revealed a decreased number of neurons in five nuclei of the complex in individuals with ASD (Kulesza et al., 2011).

In rodents, the hearing system is very well studied. In the brainstem, most of the synapses present are inhibitory and co-release GABA and glycine from the same presynaptic terminals in the superior olivary complex (Smith et al., 2000). Therefore, studying the role of NL4 in the brainstem may provide a useful model for the study of abnormalities in sensory processing in autism. For example, it is conceivable that the abnormalities in communication observed in NL4-KO mice, such as the longer response latency to female calls (Jamain et al., 2008), may be partially due to altered sensory processing. The selective vocalization impairment could potentially be explained by a different signal modulation in the hearing system of the brainstem. In the brainstem, the superior paraolivary nucleus is involved in encoding rhythmic sound patterns important for the detection of communication, such as animal vocalizations and speech signals (Felix et al., 2011). Any defect in the process of encoding a sound could lead to misinterpretation of the signal and cause the selective impairment found in NL4-KO mice. Such selective and local impairment was previously observed in the retina, in which NL4 deletion causes a subtle impairment in the visual circuit (Hoon et al., 2011).

Interestingly, NL4 protein was not detectable by immunostaining in the cerebellum (Figure 3.2F) and amygdala (Figure 3.2C-D), two brain regions that have also been implicated in the pathogenesis of autism. In the cerebellum, a wide range of morphological alterations have been reported in autistic patients (Bauman and Kemper, 2005), and in at least 
a subset of individuals with ASDs, screening of ASD gene candidates identified a number of cerebellar genes. Moreover, abnormalities involving GABA, glutamate and acetylcholine have been observed in the cerebellum of subjects with autism (Fatemi et al., 2012). The second important area devoid of NL4 staining is the amygdala, a component of the limbic system that has a primordial role in the processing and memory of emotional reactions. Disturbances in an amygdala-based network may contribute to autistic deficits in socioemotional learning and memory (Goh and Peterson, 2012).

There are several possible explanations for the apparent absence of NL4 from these autismrelated brain regions. It is conceivable that NL4 is present at levels too low to be detectable by immunostaining with the methodology described here. Alternatively, NL4 may not be relevant for those aspects of the autism phenotype that involve the cerebellum and amygdala. Given the phenotypic heterogeneity of the autism spectrum, it is very likely that different molecular mechanisms are responsible for different autism traits. Other proteins such as NL3 or SHANK2 might play a key role in the cerebellum and the amygdala instead.

\subsubsection{Correlation between localization of NL4 protein and mRNA}

One surprising aspect that arose from the global characterization of NL4 immunostaining was that the pattern of NL4 protein immunoreactivity appears to be substantially different from the pattern of NL4 mRNA expression and regional protein levels as determined previously by immunoblotting (Figure 3.1) (Jamain et al., 2008). The NL4-KO mice used in this study include a gene trap insertion within the first intron of NL4 that leads to the expression of beta-galactosidase under the control of the endogenous NL4 promoter. Using XGAL staining in these mice, it was shown that the highest level of NL4 gene expression is in olfactory bulb, striatum, cortex and hippocampus, with lower expression in the brainstem. These results were supported by immunoblotting using the same antibody as the one used here for immunohistochemistry (Jamain et al., 2008).

In contrast, immunostaining using a NL4 specific antibody revealed robust labeling in the brainstem, and lower immunoreactivity levels in olfactory bulb, striatum, cortex and hippocampus (Figure 3.1). Therefore, while the overall expression pattern of mRNA and protein is consistent, there was little correlation between the labeling intensities observed 
upon X-Gal staining in the NL4-KO and NL4 stainings. There are several possibilities to explain these findings. It is known that mRNA and protein levels do not always correlate, since protein levels can be regulated at the posttranscriptional level through regulation of local protein synthesis or protein turnover (Greenbaum et al., 2003). Moreover, the lacZ reporter gene may not entirely reflect the normal gene expression levels. The relationship between mRNA and protein immunoreactivity is very likely to be different between soluble $\mathrm{X}-\mathrm{Gal}$ and a membrane protein such as NL4. To further address this question, the localization of endogenous NL4 mRNA would need to be investigated by in situ hybridization. As regards the Western blot based NL4 localization data, the threshold level used to image specific brain regions might be too low in our immunostaining due to the low protein concentration, resulting in no detection of the protein.

\subsection{Postsynaptic architecture of NL4 positive synapses}

After identifying the regional distribution of NL4, the localization of NL4 was analyzed at the level of neuronal microarchitecture. Based on the data presented here, it appears that NL4, like the other NLs, shows a predominantly synaptic localization. It is important to note that the possibility cannot be excluded that a small fraction of NL4 might be expressed in non-neuronal cells or in extrasynaptic areas of neurons, which would explain why two types of NL4 labeling were found at high magnification, a faint and diffuse staining on the one hand versus dense punctate labeling on the other hand. However, the immunohistochemistry data presented here, showing that the majority of NL4 protein is present at synaptic structures, are in accordance with findings from a previous study, showing that NL4 was enriched in synaptic plasma membranes preparations from whole brain homogenates (Jamain et al., 2008).

A prerequisite to understanding the function of NL4 at synapses is to identify the synaptic subset that NL4 is localized to. The first key question regarding the precise synaptic localization of NL4 was to determine whether NL4 is present primarily at excitatory or inhibitory synapses. The relative proportion of excitatory and inhibitory synaptic input received by a neuron, or excitatory/inhibitory (E/I) balance, determines its level of activity, and thus its function within a given neural circuit. In recent years, a number of neuropsychiatric diseases, including ASDs and some forms of mental retardation, have been 
attributed to shifts in E/I balance (Rubenstein and Merzenich, 2003; Cornew et al., 2011). It is known that some NL isoforms localize selectively to excitatory or inhibitory synapses, with NL1 present only at excitatory synapses (Song et al., 1999) and NL2 at inhibitory synapses (Varoqueaux et al., 2004). The NL3 isoform has been shown to be present at both synapse types in vitro (Budreck and Scheiffele, 2007; Levinson et al., 2010) and an in vivo study on NL3 mutant mice demonstrated effects of the mutation on both excitatory and inhibitory synapses (Etherton et al., 2011). The particular localization of the different NLs isoforms is very likely to be associated with subtle alterations of synapse microarchitecture in the respective knockouts animals, underlining the importance of localizing the NL4 protein at the synaptic level.

In order to determine whether NL4 is localized at excitatory vs. inhibitory synapses, a selection of marker proteins was used to identify specific synaptic pathways and synaptic components. Presynaptic vesicular neurotransmitter transporters (VGluT1, VGluT2, VIAAT) were used to distinguish excitatory from inhibitory presynapses. PSD-95 was used as a marker protein to reveal the presence of NL4 at excitatory postsynapse specializations. Gephyrin served as a corresponding marker for inhibitory postsynapses. To further characterize the NL4-containing synapses, the colocalization of NL4 with postsynaptic receptors was determined, such as AMPAR (specifically GluA2), NMDAR (specifically GluN1) and $\mathrm{GABA}_{\mathrm{A}} \mathrm{R}$ (specifically the $\gamma 2$ subunit).

\subsubsection{Global immunoreactivity pattern of NL4 in relationship to gephyrin and PSD95}

One striking observation from the co-staining of NL4 with gephyrin and PSD95 was that, at low magnification, the global pattern of immunoreactivity of NL4 corresponded very closely to that of gephyrin (Figure 3.3). In contrast, in the regions of highest NL4 labeling, the globus pallidus (Figure 3.3A) and the brainstem (Figure 3.3B), PSD-95 is almost absent. These data would indicate at first glance that NL4 immunoreactivity correlates with the density of inhibitory synapses, but not the density of excitatory synapses. 


\subsubsection{NL4 in the globus pallidus and brainstem}

In the globus pallidus, NL4 clusters are preferentially colocalized with gephyrin puncta (75\%) (Figure 3.4C) and are absent from the PSD-95 puncta. In accordance with these findings, NL4 puncta are mainly apposed to VIAAT (28\%) as compared to VGluT2 (11\%), and they colocalize with $\mathrm{GABA}_{\mathrm{A}} \mathrm{R}(71 \%)$ (Figure 3.5). Similarly, in the brainstem, NL4 is present at inhibitory synapses and mainly colocalizes with gephyrin (80\%) (Figure 3.4D) and $\mathrm{GABA}_{\mathrm{A}} \mathrm{R} \gamma 2$ (55\%) (Figure 3.6D). It should be noted that the data on the VIAAT and VGluT apposition with NL4 were not entirely convincing in terms of percentage colocalization throughout this study due to experimental limitations. As described above, compromise fixation conditions were necessary for colocalization of NL4 and presynaptic markers, and under these conditions, immunostaining was sub-optimal for all markers. Nevertheless, results from this experiment provide a trend regarding the presynaptic marker apposed to NL4.

These findings are consistent with results obtained previously for NL4 localization in other brain regions. NL4, like all others NLs, contains a PDZ-binding domain (Irie et al., 1997; Poulopoulos et al., 2009) as well as a gephyrin-binding motif (Poulopoulos et al., 2009) and can therefore, in principle associate with excitatory and inhibitory postsynaptic scaffolds. However, it is known that other, more selective binding partners can determine the specific localization of individual NLs. For example, NL2 can specifically activate the small GTPase collybistin, which specifically binds to the inhibitory postsynaptic scaffold, and complexes formed by the interaction of NL2, gephyrin and collybistin are sufficient for the clustering of inhibitory neurotransmitter receptors such as $\mathrm{GABA}_{\mathrm{A}} \mathrm{R}$ (Poulopoulos et al., 2009). In the same study, it was demonstrated that NL4, but not NL1 or NL3, shares with NL2 the ability to bind to collybistin, a feature likely related to their specific role at inhibitory postsynapses in the spinal cord and the brainstem (Poulopoulos et al., 2009; Hoon et al., 2011).

In the brainstem, only half of the $\mathrm{GABA}_{\mathrm{A}} \mathrm{R}$ colocalize with NL4 whereas almost all of the NL4 puncta are localized at glycinergic synapses (Figure 3.7). The same finding was observed in different CNS regions such as the spinal cord, superior colliculus and the retina (Hoon et al., 2011). The fact that NL4 in the globus pallidus is localized purely to GABAergic synapses is presumably due to the fact that glycine receptors are absent from or only sparsely present in this region (Sato et al., 1991; Waldvogel et al., 2007). Together these data emphasize the fact that NL4, like NL2, is able to recruit and cluster GABA receptors at the 
postsynaptic membrane, but that NL4 preferentially recruits glycine receptors in some specific brains regions. NL4 might be involved in a mechanism underlying the preferential recruitment of GABA vs. glycine receptors to the inhibitory postsynaptic side. Further analysis of the brainstem and the globus pallidus would be helpful to understand the selective recruitment of the two main inhibitory receptors at the synapse.

\subsubsection{NL4 in the barrel cortex}

Based on the results described above, it was expected that NL4 would be localized at inhibitory synapses throughout the forebrain. Surprisingly, however, high magnification images of the barrel cortex showed NL4 to be mainly colocalized with PSD-95 (72\%) (Figure 3.4A) and VGluT1 (41\%) (Figure 3.7B). This result was particularly unexpected in light of the matching global immunoreactivity patterns of gephyrin and NL4 in the barrel field. PSD95 labeling in the somatosensory cortex was homogeneous in all the cortical layers, whereas gephyrin, like NL4, was present primarily in the barrel field in layer IV (Figure 3.3B). This finding emphasizes the importance of a detailed analysis of NL4 localization at the synaptic level, since the global pattern of immunoreactivity does not necessarily correlate with the local, synaptic localization of NL4 in the barrel cortex.

Unfortunately, NL4 did not colocalize with any of the excitatory postsynaptic markers that were tested. Indeed, NL4 did not colocalize with AMPAR or NMDAR (Figure 3.7E-F) or with other excitatory postsynaptic markers tested, such as metabotropic glutamate receptors, or with cell adhesion proteins specific for excitatory synapses such as leucine-rich repeat transmembrane protein 2 (LRRTM2) (Ko et al., 2009). Therefore, a detailed characterization of the synapses expressing NL4 in the barrel cortex was not possible in this context.

Layer IV of the somatosensory cortex is composed of excitatory neurons, which make numerous synaptic interconnections (Feldmeyer et al., 1999; Schubert et al., 2003), and of different subtypes of inhibitory neurons, which are known to form inhibitory networks (Gibson et al., 1999; Beierlein et al., 2000; 2003). Both excitatory and inhibitory neurons in the barrel cortex are activated by thalamocortical inputs (Agmon and Connors, 1991; 1992; Kim et al., 1995b; Castro-Alamancos and Connors, 1997; Porter et al., 2001; Beierlein et al., 2003; Murphy et al., 2012). The synaptic architecture of the barrels makes them a good area 
to study the functional involvement of NL4 at a specific synaptic microcircuit depending on sensory information.

Functional characterization of NL4 in the barrel cortex, in collaboration with the laboratory of Prof. Staiger (University of Göttingen), was recently initiated based on the observation that NL4 is present at excitatory synapses there. Preliminary experiments using patch clamp analysis in spiny stellate cells and pyramidal cells of the somatosensory cortical layer IV showed that the spontaneous activity of these cells is disturbed in NL4-KO barrel field (Prof. Jochen Staiger, personal communication). While these results do not contribute to confirming the presence of NL4 at excitatory synapses in the barrel cortex, they indicate a functional effect of NL4 deletion.

It was previously shown that another NL mutation that has been linked to ASDs, the NL3 R451C mutation, also results in an impairment of the somatosensory cortex by increasing inhibitory synaptic transmission in the layer II/III (Tabuchi et al., 2007). Neurons present in a cortical column are interconnected, and an increased inhibition in layer II/III may lead to changes in excitatory synaptic transmission in layer IV and vice versa. The identification of the localization and function of NL4 in the barrel cortex underscores the central position of the somatosensory cortex, in addition to changes in the E/I synaptic balance, in studies on ASDs. Nevertheless, the exact localization of NL4 at excitatory synapses remains to be determined.

One final possibility that remains to be explored is that NL4 could be localized at a specific subset of excitatory synapses involved in cholinergic transmission. Cholinergic neurotransmission has been implicated in neuronal plasticity (Zhu and Waite, 1998), and in the neocortex, cholinergic inputs are considered to enhance the excitability of pyramidal neurons (Krnjević et al., 1971; McCormick and Prince, 1986; Metherate et al., 1992; HajDahmane and Andrade, 1996). Thus, NL4 present at excitatory cholinergic synapses might influence the excitability of layer IV pyramidal cells, potentially leading to an increase in inhibitory transmission in layer II/III. 


\subsubsection{NL4 in the hippocampus}

Perhaps even more surprising than the localization of NL4 at excitatory synapses in barrel cortex was the observation that in the hippocampus, NL4 appears to be localized to both excitatory and inhibitory synapses. Several lines of evidence support the localization of endogenous NL4 at both glutamatergic and GABAergic synapses. Firstly, the global pattern of NL4 immunoreactivity, with strong localization in the CA1 str. l-m, corresponds to the localization of gephyrin (Figure 3.3C). While PSD-95 staining was weaker in the str. $l-m$ as compared to the str. rad, the perforant path forms excitatory synapses onto the CA1 pyramidal cells in the str. $l-m$, indicating that PSD-95 must be localized there. Secondly, at high magnification, NL4 puncta are partly colocalized with PSD-95 and partly with gephyrin (Figure 3.4B). Furthermore, in the same area, 30\% of the NL4 puncta are apposed to VGluT1, an excitatory presynapse marker (Figure 3.9B), while $42 \%$ of the NL4 clusters colocalize with $\mathrm{GABA}_{\mathrm{A}} \mathrm{R} \gamma 2$ (Figure 3.9D). Taken together, our findings indicate an association of NL4 with both GABAergic synapses and excitatory synapses. Interestingly, it has previously been proposed that NL4 in cultured hippocampal neurons is localized primarily at glutamatergic synapses (Graf et al., 2004). The data presented here expand those observations by showing that in vivo, NL4 can additionally be found at inhibitory synapses in the hippocampus. It is also important to note that the pattern of gephyrin localization corresponds to NL4 in all observed areas except for CA3, from which NL4 is absent (Figure 3.3C). NL2 might occupy the gephyrin positive clusters instead of NL4 in this particular region, although this would imply that NL2, which is exclusively present at perisomatic synapses in CA1, shows a different pattern of localization in CA3 (Poulopoulos et al., 2009).

The excitatory postsynaptic receptors present with NL4 have yet to be identified, and it is possible that NL4 might be localized to the same unknown subset of excitatory synapses as in the barrel cortex. Interestingly, the $\alpha 7$ nicotinic acetylcholine receptor subunit $(\alpha 7$ nAChRs) is present in the hippocampus, especially in the str. 1-m (Fabian-Fine et al., 2001), and double immunolabeling experiments indicate that, like NL4, $\alpha 7 \mathrm{nAChRs}$ are localized to both glutamatergic as well as GABAergic synapses (Fabian-Fine et al., 2001). In GABAergic interneurons, $\alpha 7$ nAChRs mediate cholinergic synaptic input (Frazier et al., 1998) and enhance GABAergic synapses (Frazier et al., 2003). At excitatory synapses, $\alpha 7 \mathrm{nAChRs}$ might contribute to the activation of the glutamatergic synapses and, together with the AMPAR mediate postsynaptic excitation (Levy and Aoki, 2002). The $\alpha 7$ nAChRs are 
considered as targets for cognitive enhancers (Cincotta et al., 2008) and contribute to cognitive functions study as social recognition (Van Kampen et al., 2004) and attention (Young et al., 2007). Therefore, it would be very interesting to examine the potential colocalization of $\alpha 7 \mathrm{nAChRs}$ and NL4 at hippocampal synapses and determine whether there is any connection between these two systems.

\subsection{Effect of the loss of NL4 on synapse number and distribution}

\subsubsection{Gephyrin and PSD-95 containing synapses are unchanged in the hippocampus and the barrel cortex in NL4-KO mice}

In previous studies, deletion or overexpression of various neuroligin isoforms differentially affected the composition of the postsynaptic compartment. Overexpression of NL1 in hippocampal neuron culture was shown to increase the synapse density (Chubykin et al., 2007), while NL2 deletion led to a localized decrease in gephyrin puncta around the neuronal somata (Poulopoulos et al., 2009). Based on a previous study showing that NL4 may play a role in synapse development in the retina (Hoon et al., 2011), it is reasonable to assume that NL4 may potentially play a similar role in the brain regions investigated above. To address this question, the density of excitatory and inhibitory synapses was investigated in different brains regions by comparing the density of PSD-95 and gephyrin puncta, respectively, between NL4-KO mice and WT littermates.

In the CA1 str. 1-m of the hippocampus and in layer IV of the barrel cortex, the number of PSD-95 puncta did not differ between WT and NL4-KO mice (Figure 3.10). Similarly, the number of gephyrin puncta did not differ in the barrel cortex or in the CA1 str. l-m of hippocampus, although a trend towards an increase in the density of gephyrin containing synapses was observed in the latter region (Figure 3.10). Preliminary findings from quantitative immunoblot analysis of PSD-95 and gephyrin levels in whole hippocampus and the primary somatosensory cortex indicate that there are no significant changes in the NL4-KO as compared to the WT (data not shown). These findings indicate that NL4 might not play a role in the formation of synapses in those brain regions, since alterations in synaptogenesis as a consequence of NL4 deletion should result in a change in synapse density. Alternatively, the lack of change in synapse density after the loss of NL4 may result 
from a compensatory effect of other synaptic adhesion molecules, such as others NL isoforms, as discussed below.

\subsubsection{Unchanged number of glycine receptor containing synapses in the spinal cord of NL4-KO mice}

Loss of NL4 causes a selective reduction in glycine receptor number in the retina (Hoon et al., 2011). Specifically, a 17\% decrease of the GlyR $\alpha 1$ puncta in the NL4-KO retina was observed as compared to WT (Hoon et al., 2011). This led to the question as to whether loss of NL4 may also lead to a loss of GlyR $\alpha 1$ in the CNS, where NL4 is strongly colocalized with GlyR $\alpha 1$, i.e. in the brainstem and the spinal cord (Figure 3.7) (Hoon et al., 2011).

The spinal cord contains a significant number of glycinergic synapses, especially in the lamina II of the dorsal horn, making this region particularly suitable for synapse quantification. The cells present there mainly consist of interneurons that have tiny cell bodies as compared to the large cell bodies of the motor neurons. This feature makes any irregularity in the perisomatic synapse density easier to analyze. Therefore, this region was chosen to evaluate the consequences of NL4 deletion on GlyR $\alpha 1$ puncta density.

Although NL4 puncta perfectly matched the GlyR $\alpha 1$ clusters in the WT lamina II spinal cord, no significant differences were observed between NL4-KO and WT in the glycinergic synapse density in the spinal cord (Figure 3.11). There are several possible explanations for this result. It is possible that, unlike in the retina, NL4 is not responsible for the localization of GlyR $\alpha 1$ to glycinergic synapses in the spinal cord. Alternatively, it may be that the spinal cord is a less optimal system for the detection of subtle synaptic changes than the retina. The retina is a good model to detect subtle impairments in synapse composition and function due to its highly organized laminar structure, and even under these conditions, the degree of changes observed were very small. Thus, a slight decrease of glycinergic puncta in the spinal cord might not be seen with the quantification protocol used for this study.

In summary, no changes in synapse density were observed in the NL4-KO mice in any of the CNS regions investigated. This indicates that NL4 is not required for the initial formation of synaptic contacts, but may rather play a role in synapse maturation and function. This would be in accordance with observations made in NL1-2-3 triple KOs (Varoqueaux et 
al., 2006), in which no changes in synapse density were observed but the functionality of the synapses was impaired. Unfortunately, light microscopy does not allow for the quantification of the number of receptors at synapses, which would be the measure necessary to identify a direct impact of the NL4 deletion on the functionality of synapses. On the other hand, such differences can be identified using electrophysiology, as discussed below.

\subsubsection{Functional redundancy of NLs isoforms in NL4-KO mice}

Deletion of any of the NL isoforms individually is not lethal in mice. One possible explanation for this observation may be that other NL isoforms or other synaptic adhesion proteins can compensate for the loss of any given NL isoform. In the retina, an increase in NL4 clusters was observed in NL2-KO mice, indicating that NL4 maybe able to replace NL2 in a subset of synapses that would otherwise contain NL2 alone or in combination with NL4. To test the expression levels of other NL isoforms (NL1-3) in different brain areas, total protein levels in homogenates of NL4-KOs were compared to levels in WT mice by quantitative immunoblotting. This analysis showed that NL3 and NL2 are upregulated in the primary somatosensory cortex of NL4-KOs (Figure 3.11).

The two NLs isoforms that are upregulated in NL4-KO mice are involved in inhibitory synapse formation and function, which is somewhat surprising given our finding that NL4 is present at excitatory synapses in the barrel cortex. One possible explanation could be that the deletion of NL4 does not directly result in compensatory upregulation of other NLs at the same synapses, but that the increase in NL2 and NL3 is a secondary effect of altered network activity due to impaired excitatory synaptic transmission. Decrease of the synaptic excitatory transmission would presumably decline the excitability of pyramidal cells present in layer IV, which could in turn silence interneurons that regulate the activity of other inhibitory interneurons. Thus, NL4 deletion might cause a complex network effect leading to a shift in the E/I balance in favor of inhibitory transmission, which would in turn induce the recruitment of NL isoforms present at inhibitory synapses. The fact that no difference was observed in the other brain regions of NL4-KOs might be because the barrel cortex is the only region in which NL4 was found to be mainly present at excitatory synapses. In addition, the specific synaptic organization of somatosensory cortex may allow compensatory changes to be observed that are not present in the other brain regions examined. Alternatively, other 
synaptic adhesion molecules could be involved in the compensation in response to NL4 deletion in regions other than the somatosensory cortex, or the NL4 protein concentration in these regions could be so low that any compensation that occurs would be too small to be reliably quantifiable (Varoqueaux et al., 2006). In the future, quantitative immunoblots on purified synaptosomes or synaptic plasma membrane fractions might reveal a compensatory effect of proteins levels in NL4-KO mice.

\subsection{Decreased gamma oscillations in NL4-KO mice}

A crucial function of NLs in synapse maturation appears to be to establish and maintain the balance of excitatory versus inhibitory transmission (Varoqueaux et al., 2006). An imbalance of excitatory and inhibitory transmission has emerged as a popular model to explain the functional deficits found in autism (Rubenstein and Merzenich, 2003; Levinson and El-Husseini, 2005; Murcia et al., 2005; Cornew et al., 2011). Therefore, an analysis of the effects of the loss of NL4 on the excitatory/inhibitory balance is an important step towards understanding the role of NL4 in autism-related phenotypes.

Alterations in the excitatory or in the inhibitory circuit in the hippocampus have been shown to affect the properties of gamma oscillations in this region (Fuchs et al., 2007). Therefore, the induction of gamma oscillations is a useful initial tool to study the effects of NL4 deletion on the excitatory/inhibitory balance in the hippocampus. To this end, gamma oscillations were induced in vitro by kainate application in acute brain slices under interface conditions. It was found that deletion of NL4 leads to a robust reduction of the power of hippocampal gamma oscillations (Figure 3.13), indicating that balanced excitatory and inhibitory network activity is substantially altered in these mice.

One interesting observation arising from these results is that the difference in the power of oscillations is observed in area CA3, despite the fact that NL4 levels are very low in the CA3 area of the hippocampus (Figure 3.3B). NL4 is generally weakly expressed (Varoqueaux et al., 2006), and NL4 protein expression in CA3 may simply be too low to be detected by conventional immunofluorescence staining. In support of this notion, XGAL staining in the NL4-KO revealed the NL4 gene to be expressed in the CA3 pyramidal cell layer (Jamain et al., 2008). The fact that the NL4 gene is expressed in CA3, but not highly 
enough to be detected at the protein level using immunostaining, indicates that NL4 may be involved in modulatory effects on gamma oscillations. Another possibility is that the altered oscillatory activity in the NL4-KO is the consequence of a complex response of the hippocampal circuit. NL4 is present in the CA1 str. 1-M, where the excitability of the pyramidal neurons might be modulated. Principal cells in CA1 are closely connected with an enormous variety of interneurons (Somogyi and Klausberger, 2005), and interneurons such as back-projection cells and hippocampo-septal cells can innervate CA3 (Sik et al., 1994; Freund and Buzsáki, 1996; Gulyás et al., 2003). Any modification in the synaptic transmission of these neurons might modulate a specific neuronal type in CA3 that modulates the gamma oscillations induced by kainate application.

The power of gamma oscillations in the frequency range from $25-45 \mathrm{~Hz}$ when induced with $100 \mathrm{nM}$ kainate is reduced by around 60\% in the CA3 region of NL4-KO animals as compared to gamma oscillations recorded in control slices (Figure 3.13). Interestingly, the frequency of the maximum peak in the calculated power spectrum did not change, which emphasizes the fact that the inhibitory connections are mostly intact in the NL4-KO mice (Traub et al., 2000; Atallah and Scanziani, 2009; Mann and Mody, 2010) and that the impairment observed is probably due to synaptic dysfunction. The present study did not reveal any impairment in the hippocampal synapse density of NL4-KO mice, which would indicate that there is no modification in the hippocampal connectivity of these mice. The defect observed in gamma oscillations properties could instead be explained by a defect in synapse function. A major question arising from these findings is now which synapses in the inhibitory circuitry are affected in NL4-KOs, and why. While the data presented here do not provide any direct evidence to answer this question, several potential explanations arise based on published results.

For example, it was previously shown that, like the NL4-KO mice, mice lacking the extrasynaptic subunit $\alpha 5$ of $\mathrm{GABA}_{\mathrm{A}}$ receptors also have an increase in the power of gamma oscillations in the hippocampus with no changes in the frequency (Towers et al., 2004). This may indicate a role for NL4 at a particular subset of extrasynaptic sites in the hippocampus, since $\mathrm{GABA}_{\mathrm{A}} \mathrm{R}$ subunit $\alpha 5$ is responsible for inhibition in CA3 pyramidal cells (Glykys et al., 2008). This hypothesis could be tested in the future by assessing the degree of colocalization 
of NL4 and the $\mathrm{GABA}_{\mathrm{A}} \mathrm{R} \alpha 5$ subunit and by examining the expression and localization of $\mathrm{GABA}_{\mathrm{A}} \mathrm{R} \alpha 5$ in NL4-KO hippocampus.

Another possibility is that NL4 deletion leads to a reduction of the GABA-mediated response from PV-positive interneurons. PV-positive interneurons in the hippocampus are implicated in the generation and regulation of gamma oscillations in vitro (Gulyás et al., 2010). PV-expressing neurons include basket cells and axo-axonic cells, which inhibit the axon initial segment of pyramidal cells (Gloveli et al., 2005; Gulyás et al., 2010),(Hájos et al., 2004). PV-positive basket cells form perisomatic synapses onto pyramidal cells, and perisomatic feedback inhibition onto pyramidal cells has been implicated in the generation of gamma oscillations (Mann et al., 2005a). Thus, PV basket cells are in a perfect position to regulate the activity of pyramidal cells (Freund and Katona, 2007). The network connections formed between PV positive basket cells, combined with their diverse innervation of pyramidal neurons, allows them to spread neuronal activity within a cellular network (Cobb et al., 1995; Sik et al., 1995; Gulyás et al., 1999; Bartos et al., 2007). If NL4 were positioned in a manner to affect the physiological properties of these PV basket cells or their downstream target neurons, this would explain the effect of NL4 deletion on gamma oscillations in the hippocampus.

Together with PV basket cells, Cholecystokinin (CCK)-expressing basket cells provide perisomatic inhibition to pyramidal cells (Freund and Katona, 2007). In contrast, to PV cells, CCK-positive cells have been suggested to provide a modulatory input, rather than supplying the driving force for the induction of gamma oscillations (Freund and Katona, 2007). In the NL4-KO mice, kainate-induced gamma oscillations are decreased but not abolished. The same result was found in response to application of an agonist of the CB1 receptor, which reduced the power of gamma oscillations dramatically (Hájos et al., 2000). The CB1 receptor is exclusively expressed by CCK positive cells and not by the PV containing cells (Katona et al., 1999; Marsicano and Lutz, 1999), which can be taken as evidence for a more prominent role of the CCK basket cells in generating the NL4-KO phenotype described here. However, it should be noted that the reduction in gamma oscillation power in response to the $\mathrm{CB} 1$ receptor agonist might also be due to the presence of CB1 receptors on pyramidal cells, which causes the inhibition of glutamate release (Marsicano and Lutz, 1999; Domenici et al., 2006; Katona et al., 2006). 
Interestingly, it has been reported that CCK containing basket cells in the hippocampus participate in the generation of gamma oscillations in response to kainate application, but not in response to cholinergic stimulation in the hippocampus in vitro (Gulyás et al., 2010). Therefore, if CCK-positive interneurons play a role in the NL4-KO phenotype observed for the kainate-induced gamma oscillations, one would expect there to be no effect of NL4 deletion on gamma oscillations induced by cholinergic stimulation.

Gamma oscillations induced by kainate application have been shown to correlate with gamma oscillations produced in vivo during spatial reference memory tests (Lu et al., 2011). Specifically, kainate-induced oscillations correlate with in vivo recording of local field potentials during Barnes circular platform performance and object location recognition, and in vitro oscillations recordings were larger in mice that recognized the aversive context in a passive avoidance task ( $\mathrm{Lu}$ et al., 2011). It will be interesting to determine whether the oscillation phenotype found in the NL4-KO mice leads to alterations in spatial reference memory in tasks such as those described above, which would provide a useful model system for studying the mechanisms underlying learning impairments in ASDs. 


\section{Summary and Conclusions}

Neuroligins (NLs) are postsynaptic cell adhesion proteins involved in synaptogenesis and synapse maturation. This study analyzed the distribution and the function of one NL isoform, NL4. The distribution of NL4 was characterized throughout the central nervous system, together with the synaptic composition of NL4-containing synapses in the relevant brain regions. The role of NL4 in the maintenance of the synaptic circuitry was investigated by analyzing the density of excitatory and inhibitory synapses in NL4-KO mice. In addition, potential compensatory effects by other NL isoforms in response to the deletion of NL4 were analyzed in selected brain regions. Finally, functional studies were performed to characterize the contribution of NL4 in the generation of oscillatory activity in the hippocampus.

NL4 is distributed ubiquitously in the brainstem, spinal cord and globus pallidus, and locally in the cortex and the hippocampus. At the synaptic level, NL4 is present at excitatory and inhibitory synapses depending on the brain region. In the globus pallidus, spinal cord and the brainstem, NL4 is present at inhibitory postsynapses, localizing preferentially to glycinergic synapses in the brainstem and the spinal cord and exclusively to GABAergic synapses in the globus pallidus. Strikingly, NL4 is localized to both inhibitory and excitatory synapses in the hippocampus. In the barrel cortex, NL4 was found to mainly localize to excitatory synapses. Loss of NL4 is not accompanied by a modification of the synapse density in the hippocampus, barrel cortex or spinal cord. However, a local, potentially compensatory, upregulation of NL2 and NL3 was observed in the barrel cortex of NL4-KO mice. Finally, the deletion of NL4 induced a selective impairment in gamma oscillations generated by kainate in the hippocampus.

Together, these data show that NL4 immunoreactivity is present throughout the CNS in brains regions related to ASD, confirming the relevance of studying this protein in the context of neurodevelopmental disorders. The synaptic localization of NL4 was found to be dependent on the specific brain region, reflecting a complex role of the protein at the synapse, although the functional mechanisms remain unknown. Studies on protein function using the 
NL4-KO revealed that NL4 is necessary for the integrity of local neural circuits, and hence for system homeostasis. Any defects in this homeostasis may disturb the balance of excitatory and inhibitory transmission, an effect that has been reported in numerous cases of ASD. 


\section{Outlook}

This study is the first characterization of the NL4 distribution and function in the central nervous system, and reveals diverse roles of the protein throughout the central nervous system. However, a number of questions remain to be addressed in order to fully understand the role of NL4 in synapse development and function, as well as its relevance to ASD.

In order to confirm the exact localization of NL4 in the barrel cortex and the hippocampus, it will be necessary to raise new antibodies targeted against NL4 so as to perform better light microscopy and immunogold studies electron microscopy in those brains regions. These experiments were attempted in the context of this thesis work using the only specific antibody available in our laboratory (and in the world), but the fixation conditions necessary for the NL4 antibody were not compatible with those required for light microscopy and electron microscopy experiments in the barrel cortex and hippocampus. Immunogold labeling and electron microscopic analyzes will be necessary to confirm the exact localization of NL4 at excitatory (type 1) or inhibitory synapses (type 2) in the barrel cortex and the hippocampus. Such data would then guide further analysis by electrophysiology.

In the hippocampus, NL4 immunoreactivity was found in the str. $l-m$, and alterations in the power of gamma oscillations were observed in the NL4-KO mice, reflecting a selective impairment of the hippocampal neural network. An important future experiment will be to analyze the precise role of NL4 in the O-LM-pyramidal cell circuit in area CA1. One method to achieve this would be to conduct patch clamp recordings such as paired recordings of one pyramidal cell and one inhibitory interneuron. Extracellular stimulation of the perforant path, combined with recording of the O-LM interneurons or the CA1 pyramidal neurons, would be interesting to determine the effect of loss of NL4 on synapse function in this region, and to investigate whether these changes play any role in the genesis of autism-related behavioral phenotypes. O-LM cells do not fire phase locked to gamma oscillations, and therefore they may not contribute to the generation of gamma oscillations. However, they fire at the trough of the theta cycle (Klausberger et al., 2003), which could implicate NL4 in an as yet unknown oscillatory system. 


\section{References}

Agmon A, Connors BW (1991) Thalamocortical responses of mouse somatosensory (barrel) cortex in vitro. NSC 41:365-379.

Agmon A, Connors BW (1992) Correlation between intrinsic firing patterns and thalamocortical synaptic responses of neurons in mouse barrel cortex. J Neurosci 12:319329.

Ali AB, Thomson AM (1998) Facilitating pyramid to horizontal oriens-alveus interneurone inputs: dual intracellular recordings in slices of rat hippocampus. J Physiol (Lond) 507 ( Pt 1):185-199.

Arac D, Boucard AA, Ozkan E, Strop P, Newell E, Sudhof TC, Brunger AT (2007) Structures of neuroligin-1 and the neuroligin-1/neurexin- 1 beta complex reveal specific proteinprotein and protein-Ca2+ interactions. Neuron 56:992-1003.

Arstikaitis P, Gauthier-Campbell C, Huang K, El-Husseini A, Murphy TH (2011) Proteins that promote filopodia stability, but not number, lead to more axonal-dendritic contacts. Plos One 6:e16998.

Asperger H (1944) Asperger: Die Autistischen Psychopaten in Kindesalter.

Atallah BV, Scanziani M (2009) Instantaneous modulation of gamma oscillation frequency by balancing excitation with inhibition. Neuron 62:566-577.

Baio J (2012) Prevalence of Autism Spectrum Disorders-Autism and Developmental Disabilities Monitoring Network, 14 Sites, United States, 2008. cdcgov

Barrow SL, Constable JR, Clark E, El-Sabeawy F, McAllister AK, Washbourne P (2009) Neuroligin1: a cell adhesion molecule that recruits PSD-95 and NMDA receptors by distinct mechanisms during synaptogenesis. Neural Dev 4:17.

Bartos M, Vida I, Jonas P (2007) Synaptic mechanisms of synchronized gamma oscillations in inhibitory interneuron networks. Nat Rev Neurosci 8:45-56.

Baudouin S, Scheiffele P (2010) SnapShot: Neuroligin-neurexin complexes. Cell 141:908, 908.e1.

Bauman ML, Kemper TL (2003) The neuropathology of the autism spectrum disorders: what have we learned? Novartis Found Symp 251:112-22-discussion122-8-281-97.

Bauman ML, Kemper TL (2005) Neuroanatomic observations of the brain in autism: a review and future directions. Int J Dev Neurosci 23:183-187. 
Beierlein M, Gibson JR, Connors BW (2000) A network of electrically coupled interneurons drives synchronized inhibition in neocortex. Nature Neuroscience 3:904-910.

Beierlein M, Gibson JR, Connors BW (2003) Two dynamically distinct inhibitory networks in layer 4 of the neocortex. Journal of Neurophysiology 90:2987-3000.

Berkel S, Marshall CR, Weiss B, Howe J, Roeth R, Moog U, Endris V, Roberts W, Szatmari P, Pinto D, Bonin M, Riess A, Engels H, Sprengel R, Scherer SW, Rappold GA (2010) Mutations in the SHANK2 synaptic scaffolding gene in autism spectrum disorder and mental retardation. Nature Publishing Group 42:489-491.

Blundell J, Blaiss CA, Etherton MR, Espinosa F, Tabuchi K, Walz C, Bolliger MF, Sudhof TC, Powell CM (2010) Neuroligin-1 deletion results in impaired spatial memory and increased repetitive behavior. Journal of Neuroscience 30:2115-2129.

Bolliger MF, Pei J, Maxeiner S, Boucard AA, Grishin NV, Sudhof TC (2008) Unusually rapid evolution of Neuroligin-4 in mice. Proc Natl Acad Sci USA 105:6421-6426.

Boucard AA, Chubykin AA, Comoletti D, Taylor P, Sudhof TC (2005) A splice code for trans-synaptic cell adhesion mediated by binding of neuroligin 1 to alpha- and betaneurexins. Neuron 48:229-236.

Bourgeron T (2009) A synaptic trek to autism. Current Opinion in neurobiology 19:231-234.

Bragin A, Jandó G, Nádasdy Z, Hetke J, Wise K, Buzsáki G (1995) Gamma (40-100Hz) oscillation in the hippocampus of the behaving rat. J Neurosci 15:47-60.

Brecht M, Preilowski B, Merzenich MM (1997) Functional architecture of the mystacial vibrissae. Behav Brain Res 84:81-97.

Budreck EC, Scheiffele P (2007) Neuroligin-3 is a neuronal adhesion protein at GABAergic and glutamatergic synapses. Eur J Neurosci 26:1738-1748.

Butz S, Okamoto M, Sudhof TC (1998) A tripartite protein complex with the potential to couple synaptic vesicle exocytosis to cell adhesion in brain. Cell 94:773-782.

Buxbaum JD (2009) Multiple rare variants in the etiology of autism spectrum disorders. Dialogues Clin Neurosci 11:35-43.

Buxhoeveden DP, Semendeferi K, Buckwalter J, Schenker N, Switzer R, Courchesne E (2006) Reduced minicolumns in the frontal cortex of patients with autism. Neuropathol Appl Neurobiol 32:483-491.

Cascio CJ, Moana-Filho EJ, Guest S, Nebel MB, Weisner J, Baranek GT, Essick GK (2012) Perceptual and Neural Response to Affective Tactile Texture Stimulation in Adults with Autism Spectrum Disorders. Autism Res.

Castro-Alamancos MA, Connors BW (1997) Thalamocortical synapses. Prog Neurobiol 51:581-606.

Chen L, Chetkovich DM, Petralia RS, Sweeney NT, Kawasaki Y, Wenthold RJ, Bredt DS, Nicoll RA (2000) Stargazin regulates synaptic targeting of AMPA receptors by two 
distinct mechanisms. Nature 408:936-943.

Chen SX, Tari PK, She K, Haas K (2010) Neurexin-neuroligin cell adhesion complexes contribute to synaptotropic dendritogenesis via growth stabilization mechanisms in vivo. Neuron 67:967-983.

Chen X, Liu H, Shim AHR, He PJFX (2007) Structural basis for synaptic adhesion mediated by neuroligin-neurexin interactions. NATURE STRUCTURAL \& MOLECULAR BIOLOGY:1-7.

Chéry N, de Koninck Y (1999) Junctional versus extrajunctional glycine and GABA(A) receptor-mediated IPSCs in identified lamina I neurons of the adult rat spinal cord. Journal of Neuroscience 19:7342-7355.

Chih B, Afridi SK, Clark L, Scheiffele P (2004) Disorder-associated mutations lead to functional inactivation of neuroligins. Human Molecular Genetics 13:1471-1477.

Chubykin AA, Atasoy D, Etherton MR, Brose N, Kavalali ET, Gibson JR, Sudhof TC (2007) Activity-dependent validation of excitatory versus inhibitory synapses by neuroligin-1 versus neuroligin-2. Neuron 54:919-931.

Chubykin AA, Liu X, Comoletti D, Tsigelny I, Taylor P, Sudhof TC (2005) Dissection of synapse induction by neuroligins: effect of a neuroligin mutation associated with autism. J Biol Chem 280:22365-22374.

Cincotta SL, Yorek MS, Moschak TM, Lewis SR, Rodefer JS (2008) Selective nicotinic acetylcholine receptor agonists: potential therapies for neuropsychiatric disorders with cognitive dysfunction. Curr Opin Investig Drugs 9:47-56.

Cobb SR, Buhl EH, Halasy K, Paulsen O, Somogyi P (1995) Synchronization of neuronal activity in hippocampus by individual GABAergic interneurons. Nature 378:75-78.

Colbert CM, Levy WB (1992) Electrophysiological and pharmacological characterization of perforant path synapses in CA1: mediation by glutamate receptors. Journal of Neurophysiology 68:1-8.

Comoletti D, De Jaco A, Jennings LL, Flynn RE, Gaietta G, Tsigelny I, Ellisman MH, Taylor $P$ (2004) The Arg451Cys-neuroligin-3 mutation associated with autism reveals a defect in protein processing. Journal of Neuroscience 24:4889-4893.

Comoletti D, Flynn R, Jennings LL, Chubykin A, Matsumura T, Hasegawa H, Sudhof TC, Taylor P (2003) Characterization of the interaction of a recombinant soluble neuroligin-1 with neurexin-1beta. J Biol Chem 278:50497-50505.

Comoletti D, Grishaev A, Whitten AE, Tsigelny I, Taylor P, Trewhella J (2007) Synaptic arrangement of the neuroligin/beta-neurexin complex revealed by X-ray and neutron scattering. Structure 15:693-705.

Cornew L, Roberts TPL, Blaskey L, Edgar JC (2011) Resting-State Oscillatory Activity in Autism Spectrum Disorders. J Autism Dev Disord.

Courchesne E, Carper R, Akshoomoff N (2003) Evidence of brain overgrowth in the first year 
of life in autism. JAMA 290:337-344.

Courchesne E, Pierce K, Schumann CM, Redcay E, Buckwalter JA, Kennedy DP, Morgan J (2007) Mapping early brain development in autism. Neuron 56:399-413.

Couteaux R, Pécot-Dechavassine M (1973) [Ultrastructural and cytochemical data on the mechanism of acetylcholine release in synaptic transmission]]. Arch Ital Biol 111:231262.

Craig AM, Kang Y (2007) Neurexin-neuroligin signaling in synapse development. Current Opinion in neurobiology 17:43-52.

Dahlhaus R, Hines RM, Eadie BD, Kannangara TS, Hines DJ, Brown CE, Christie BR, ElHusseini A (2010) Overexpression of the cell adhesion protein neuroligin-1 induces learning deficits and impairs synaptic plasticity by altering the ratio of excitation to inhibition in the hippocampus. Hippocampus 20:305-322.

Dean C, Scholl FG, Choih J, DeMaria S, Berger J, Isacoff E, Scheiffele P (2003) Neurexin mediates the assembly of presynaptic terminals. Nature Neuroscience 6:708-716.

Diamond ME, Heimendahl von M, Arabzadeh E (2008) Whisker-mediated texture discrimination. PLoS Biol 6:e220.

Difiglia M, Rafols JA (1988) Synaptic Organization of the Globus Pallidus. J Electron Microsc Tech:1-17.

Domenici MR, Azad SC, Marsicano G, Schierloh A, Wotjak CT, Dodt H-U, Zieglgänsberger W, Lutz B, Rammes G (2006) Cannabinoid receptor type 1 located on presynaptic terminals of principal neurons in the forebrain controls glutamatergic synaptic transmission. Journal of Neuroscience 26:5794-5799.

Dong N, Qi J, Chen G (2007) Molecular reconstitution of functional GABAergic synapses with expression of neuroligin-2 and GABAA receptors. Mol Cell Neurosci 35:14-23.

Dölen G, Bear MF (2008) Role for metabotropic glutamate receptor 5 (mGluR5) in the pathogenesis of fragile X syndrome. J Physiol (Lond) 586:1503-1508.

Durand CM et al. (2007) Mutations in the gene encoding the synaptic scaffolding protein SHANK3 are associated with autism spectrum disorders. Nat Genet 39:25-27.

Empson RM, Heinemann U (1995a) Perforant path connections to area CA1 are predominantly inhibitory in the rat hippocampal-entorhinal cortex combined slice preparation. Hippocampus 5:104-107.

Empson RM, Heinemann U (1995b) The perforant path projection to hippocampal area CA1 in the rat hippocampal-entorhinal cortex combined slice. J Physiol (Lond) 484 ( Pt 3):707-720.

Essrich C, Lorez M, Benson JA, Fritschy J-M, Lüscher B (1998) Postsynaptic clustering of major GABAA receptor subtypes requires the gamma 2 subunit and gephyrin. Nature Neuroscience 1:563-571. 
Estes A, Shaw DWW, Sparks BF, Friedman S, Giedd JN, Dawson G, Bryan M, Dager SR (2011) Basal ganglia morphometry and repetitive behavior in young children with autism spectrum disorder. Autism Res 4:212-220.

Etherton M, Földy C, Sharma M, Tabuchi K, Liu X, Shamloo M, Malenka RC, Sudhof TC (2011) Autism-linked neuroligin-3 R451C mutation differentially alters hippocampal and cortical synaptic function. Proc Natl Acad Sci USA 108:13764-13769.

Etherton MR, Blaiss CA, Powell CM, Sudhof TC (2009) Mouse neurexin-1alpha deletion causes correlated electrophysiological and behavioral changes consistent with cognitive impairments. Proc Natl Acad Sci USA 106:17998-18003.

Fabian-Fine R, Skehel P, Errington ML, Davies HA, Sher E, Stewart MG, Fine A (2001) Ultrastructural distribution of the alpha7 nicotinic acetylcholine receptor subunit in rat hippocampus. Journal of Neuroscience 21:7993-8003.

Fabrichny IP, Leone P, Sulzenbacher G, Comoletti D, Miller MT, Taylor P, Bourne Y, Marchot P (2007) Structural analysis of the synaptic protein neuroligin and its betaneurexin complex: determinants for folding and cell adhesion. Neuron 56:979-991.

Falls WM, Park MR, Kitai ST (1983) An intracellular HRP study of the rat globus pallidus. II. Fine structural characteristics and synaptic connections of medially located large GP neurons. J Comp Neurol 221:229-245.

Fatemi SH et al. (2012) Consensus Paper: Pathological Role of the Cerebellum in Autism. Cerebellum.

Feldmeyer D, Egger V, Lübke J, Sakmann B (1999) Reliable synaptic connections between pairs of excitatory layer 4 neurones within a single "barrel" of developing rat somatosensory cortex. J Physiol (Lond) 521 Pt 1:169-190.

Felix RA, Fridberger A, Leijon S, Berrebi AS, Magnusson AK (2011) Sound rhythms are encoded by postinhibitory rebound spiking in the superior paraolivary nucleus. Journal of Neuroscience 31:12566-12578.

Fisahn A, Contractor A, Traub RD, Buhl EH, Heinemann SF, McBain CJ (2004) Distinct roles for the kainate receptor subunits GluR5 and GluR6 in kainate-induced hippocampal gamma oscillations. Journal of Neuroscience 24:9658-9668.

Fisahn A, Pike FG, Buhl EH, Paulsen O (1998) Cholinergic induction of network oscillations at $40 \mathrm{~Hz}$ in the hippocampus in vitro. Nature 394:186-189.

Fisahn A, Yamada M, Duttaroy A, Gan J-W, Deng C-X, McBain CJ, Wess J (2002) Muscarinic induction of hippocampal gamma oscillations requires coupling of the M1 receptor to two mixed cation currents. Neuron 33:615-624.

Franklin GPKBJ (2001) The Mouse Brain in Stereotaxic Coordinates. :1-350.

Frazier CJ, Rollins YD, Breese CR, Leonard S, Freedman R, Dunwiddie TV (1998) Acetylcholine activates an alpha-bungarotoxin-sensitive nicotinic current in rat hippocampal interneurons, but not pyramidal cells. J Neurosci 18:1187-1195. 
Frazier CJ, Strowbridge BW, Papke RL (2003) Nicotinic receptors on local circuit neurons in dentate gyrus: a potential role in regulation of granule cell excitability. Journal of Neurophysiology 89:3018-3028.

Freund TF (2003) Interneuron Diversity series: Rhythm and mood in perisomatic inhibition. TRENDS in Neurosciences 26:489-495.

Freund TF, Buzsáki G (1996) Interneurons of the hippocampus. Hippocampus 6:347-470.

Freund TF, Katona I (2007) Perisomatic inhibition. Neuron 56:33-42.

Fritschy J-M, Brünig I (2003) Formation and plasticity of GABAergic synapses: physiological mechanisms and pathophysiological implications. Pharmacol Ther 98:299_ 323.

Fritschy J-M, Mohler H (1995) GABAA-receptor heterogeneity in the adult rat brain: differential regional and cellular distribution of seven major subunits. J Comp Neurol 359:154-194.

Fu Z, Vicini S (2009) Neuroligin-2 accelerates GABAergic synapse maturation in cerebellar granule cells. Mol Cell Neurosci 42:45-55.

Fuchs EC, Zivkovic AR, Cunningham MO, Middleton S, Lebeau FEN, Bannerman DM, Rozov A, Whittington MA, Traub RD, Rawlins JNP, Monyer H (2007) Recruitment of parvalbumin-positive interneurons determines hippocampal function and associated behavior. Neuron 53:591-604.

GARNER CC, Zhai RG, GUNDELFINGER ED, Ziv NE (2002) Molecular mechanisms of CNS synaptogenesis. TRENDS in Neurosciences 25:243-251.

Gauthier J et al. (2011) Truncating mutations in NRXN2 and NRXN1 in autism spectrum disorders and schizophrenia. Hum Genet 130:563-573.

Gauthier J, Bonnel A, St-Onge J, Karemera L, Laurent S, Mottron L, Fombonne E, Joober R, Rouleau GA (2005) NLGN3/NLGN4 gene mutations are not responsible for autism in the Quebec population. Am J Med Genet B Neuropsychiatr Genet 132B:74-75.

Gerrow K, El-Husseini A (2006) Cell adhesion molecules at the synapse. Front Biosci 11:2400-2419.

Gibson JR, Beierlein M, Connors BW (1999) Two networks of electrically coupled inhibitory neurons in neocortex. Nature 402:75-79.

Gibson JR, Huber KM, Sudhof TC (2009) Neuroligin-2 deletion selectively decreases inhibitory synaptic transmission originating from fast-spiking but not from somatostatinpositive interneurons. Journal of Neuroscience 29:13883-13897.

Gillies MJ, Traub RD, LeBeau FEN, Davies CH, Gloveli T, Buhl EH, Whittington MA (2002) A model of atropine-resistant theta oscillations in rat hippocampal area CA1. J Physiol (Lond) 543:779-793.

Glickfeld LL, Scanziani M (2006) Distinct timing in the activity of cannabinoid-sensitive and 
cannabinoid-insensitive basket cells. Nature Neuroscience 9:807-815.

Gloveli T, Dugladze T, Saha S, Monyer H, Heinemann U, Traub RD, Whittington MA, Buhl EH (2005) Differential involvement of oriens/pyramidale interneurones in hippocampal network oscillations in vitro. J Physiol (Lond) 562:131-147.

Glykys J, Mann EO, Mody I (2008) Which GABA(A) receptor subunits are necessary for tonic inhibition in the hippocampus? Journal of Neuroscience 28:1421-1426.

Goh S, Peterson BS (2012) Imaging evidence for disturbances in multiple learning and memory systems in persons with autism spectrum disorders. Dev Med Child Neurol 54:208-213.

Graf ER, Zhang X, Jin S-X, Linhoff MW, Craig AM (2004) Neurexins induce differentiation of GABA and glutamate postsynaptic specializations via neuroligins. Cell 119:10131026.

Gray EG (1959) Axo-somatic and axo-dendritic synapses of the cerebral cortex: an electron microscope study. J Anat 93:420-433.

Greenbaum D, Colangelo C, Williams K, Gerstein M (2003) Comparing protein abundance and mRNA expression levels on a genomic scale. Genome Biol 4:117.

Gulyás AI, Hájos N, Katona I, Freund TF (2003) Interneurons are the local targets of hippocampal inhibitory cells which project to the medial septum. Eur J Neurosci 17:1861-1872.

Gulyás AI, Megías M, Emri Z, Freund TF (1999) Total number and ratio of excitatory and inhibitory synapses converging onto single interneurons of different types in the CA1 area of the rat hippocampus. Journal of Neuroscience 19:10082-10097.

Gulyás AI, Szabó GG, Ulbert I, Holderith N, Monyer H, Erdélyi F, Szabó G, Freund TF, Hájos N (2010) Parvalbumin-containing fast-spiking basket cells generate the field potential oscillations induced by cholinergic receptor activation in the hippocampus. Journal of Neuroscience 30:15134-15145.

Haj-Dahmane S, Andrade R (1996) Muscarinic activation of a voltage-dependent cation nonselective current in rat association cortex. J Neurosci 16:3848-3861.

Halpain S, Spencer K, Graber S (2005) Dynamics and pathology of dendritic spines. Prog Brain Res 147:29-37.

Harvey RJ, Depner UB, Wässle H, Ahmadi S, Heindl C, Reinold H, Smart TG, Harvey K, Schütz B, Abo-Salem OM, Zimmer A, Poisbeau P, Welzl H, Wolfer DP, Betz H, Zeilhofer HU, Müller U (2004) GlyR alpha3: an essential target for spinal PGE2mediated inflammatory pain sensitization. Science 304:884-887.

Hazlett HC, Poe M, Gerig G, Smith RG, Provenzale J, Ross A, Gilmore J, Piven J (2005) Magnetic resonance imaging and head circumference study of brain size in autism: birth through age 2 years. Arch Gen Psychiatry 62:1366-1376.

Hájos N, Katona I, Naiem SS, Mackie K, Ledent C, Mody I, Freund TF (2000) Cannabinoids 
inhibit hippocampal GABAergic transmission and network oscillations. Eur J Neurosci 12:3239-3249.

Hájos N, Paulsen O (2009) Network mechanisms of gamma oscillations in the CA3 region of the hippocampus. Neural Networks 22:1113-1119.

Hájos N, Pálhalmi J, Mann EO, Németh B, Paulsen O, Freund TF (2004) Spike timing of distinct types of GABAergic interneuron during hippocampal gamma oscillations in vitro. Journal of Neuroscience 24:9127-9137.

Heine M, Thoumine O, Mondin M, Tessier B, Giannone G, Choquet D (2008) Activityindependent and subunit-specific recruitment of functional AMPA receptors at neurexin/neuroligin contacts. Proc Natl Acad Sci USA 105:20947-20952.

Herbert MR (2010) Contributions of the environment and environmentally vulnerable physiology to autism spectrum disorders. Curr Opin Neurol 23:103-110.

Hines RM, Wu L, Hines DJ, Steenland H, Mansour S, Dahlhaus R, Singaraja RR, Cao X, Sammler E, Hormuzdi SG, Zhuo M, El-Husseini A (2008) Synaptic imbalance, stereotypies, and impaired social interactions in mice with altered neuroligin 2 expression. Journal of Neuroscience 28:6055-6067.

Hirao K, Hata Y, Ide N, Takeuchi M, Irie M, Yao I, Deguchi M, Toyoda A, Sudhof TC, Takai Y (1998) A novel multiple PDZ domain-containing molecule interacting with N-methylD-aspartate receptors and neuronal cell adhesion proteins. J Biol Chem 273:2110521110.

Hoon M, Bauer G, FRITSCHY J-M, Moser T, Falkenburger BH, Varoqueaux F (2009) Neuroligin 2 controls the maturation of GABAergic synapses and information processing in the retina. Journal of Neuroscience 29:8039-8050.

Hoon M, Soykan T, Falkenburger B, Hammer M, Patrizi A, Schmidt K-F, Sassoè-Pognetto M, Löwel S, Moser T, Taschenberger H, Brose N, Varoqueaux F (2011) Neuroligin-4 is localized to glycinergic postsynapses and regulates inhibition in the retina. Proc Natl Acad Sci USA 108:3053-3058.

Ichtchenko K, Hata Y, Nguyen T, Ullrich B, Missler M, Moomaw C, Sudhof TC (1995) Neuroligin 1: a splice site-specific ligand for beta-neurexins. Cell 81:435-443.

Ichtchenko K, Nguyen T, Sudhof TC (1996) Structures, alternative splicing, and neurexin binding of multiple neuroligins. J Biol Chem 271:2676-2682.

Irie M, Hata Y, Takeuchi M, Ichtchenko K, Toyoda A, Hirao K, Takai Y, Rosahl TW, Sudhof TC (1997) Binding of neuroligins to PSD-95. Science 277:1511-1515.

Jamain S, Quach H, Betancur C, Råstam M, Colineaux C, Gillberg IC, Soderstrom H, Giros B, Leboyer M, Gillberg C, Bourgeron T, Paris Autism Research International Sibpair Study (2003) Mutations of the X-linked genes encoding neuroligins NLGN3 and NLGN4 are associated with autism. Nat Genet 34:27-29.

Jamain S, Radyushkin K, Hammerschmidt K, Granon S, Boretius S, Varoqueaux F, Ramanantsoa N, Gallego J, Ronnenberg A, Winter D, Frahm J, Fischer J, Bourgeron T, 
Ehrenreich H, Brose N (2008) Reduced social interaction and ultrasonic communication in a mouse model of monogenic heritable autism. Proc Natl Acad Sci USA 105:17101715.

Jedlicka P, Hoon M, Papadopoulos T, Vlachos A, Winkels R, Poulopoulos A, Betz H, Deller T, Brose N, Varoqueaux F, Schwarzacher SW (2011) Increased dentate gyrus excitability in neuroligin-2-deficient mice in vivo. Cereb Cortex 21:357-367.

Jonas P, Bischofberger J, Fricker D, Miles R (2004) Interneuron Diversity series: Fast in, fast out--temporal and spatial signal processing in hippocampal interneurons. TRENDS in Neurosciences 27:30-40.

Jung S-Y, Kim J, Kwon OB, Jung JH, An K, Jeong AY, Lee CJ, Choi Y-B, Bailey CH, Kandel ER, Kim J-H (2010) Input-specific synaptic plasticity in the amygdala is regulated by neuroligin-1 via postsynaptic NMDA receptors. Proc Natl Acad Sci USA 107:4710-4715.

Just MA, Cherkassky VL, Keller TA, Kana RK, Minshew NJ (2006) Functional and Anatomical Cortical Underconnectivity in Autism: Evidence from an fMRI Study of an Executive Function Task and Corpus Callosum Morphometry. Cereb Cortex 17:951-961.

Kalscheuer VM, Musante L, Fang C, Hoffmann K, Fuchs C, Carta E, Deas E, Venkateswarlu K, Menzel C, Ullmann R, Tommerup N, Dalprà L, Tzschach A, Selicorni A, Luscher B, Ropers H-H, Harvey K, Harvey RJ (2009) A balanced chromosomal translocation disrupting ARHGEF9 is associated with epilepsy, anxiety, aggression, and mental retardation. Hum Mutat 30:61-68.

Kanner L (1943) Child psychiatry - Mental deficiency. Am J Psychiat 99:608-610.

Katona I, Sperlágh B, Sik A, Käfalvi A, Vizi ES, Mackie K, Freund TF (1999)

Presynaptically located CB1 cannabinoid receptors regulate GABA release from axon terminals of specific hippocampal interneurons. Journal of Neuroscience 19:4544-4558.

Katona I, Urbán GM, Wallace M, Ledent C, Jung K-M, Piomelli D, Mackie K, Freund TF (2006) Molecular composition of the endocannabinoid system at glutamatergic synapses. Journal of Neuroscience 26:5628-5637.

Kim E, Niethammer M, Rothschild A, Jan YN, Sheng M (1995a) Clustering of Shaker-type $\mathrm{K}+$ channels by interaction with a family of membrane-associated guanylate kinases. Nature 378:85-88.

Kim H-G et al. (2008) Disruption of neurexin 1 associated with autism spectrum disorder. Am J Hum Genet 82:199-207.

Kim HG, Fox K, Connors BW (1995b) Properties of excitatory synaptic events in neurons of primary somatosensory cortex of neonatal rats. Cereb Cortex 5:148-157.

Kins S, Betz H, Kirsch J (2000) Collybistin, a newly identified brain-specific GEF, induces submembrane clustering of gephyrin. Nature Neuroscience 3:22-29.

Kita H, Kitai ST (1987) Efferent projections of the subthalamic nucleus in the rat: light and electron microscopic analysis with the PHA-L method. J Comp Neurol 260:435-452. 
Klausberger T, Magill PJ, Márton LF, Roberts JDB, Cobden PM, Buzsáki G, Somogyi P (2003) Brain-state- and cell-type-specific firing of hippocampal interneurons in vivo. Nature 421:844-848.

Kneussel M, Brandstätter JH, Laube B, Stahl S, Müller U, Betz H (1999) Loss of postsynaptic $\mathrm{GABA}(\mathrm{A})$ receptor clustering in gephyrin-deficient mice. Journal of Neuroscience 19:9289-9297.

Ko J, Fuccillo MV, Malenka RC, Sudhof TC (2009) LRRTM2 functions as a neurexin ligand in promoting excitatory synapse formation. Neuron 64:791-798.

Kolevzon A, Gross R, Reichenberg A (2007) Prenatal and perinatal risk factors for autism: a review and integration of findings. Arch Pediatr Adolesc Med 161:326-333.

Kornau HC, Schenker LT, Kennedy MB, Seeburg PH (1995) Domain interaction between NMDA receptor subunits and the postsynaptic density protein PSD-95. Science 269:1737-1740.

Krnjević K, Pumain R, Renaud L (1971) The mechanism of excitation by acetylcholine in the cerebral cortex. J Physiol (Lond) 215:247-268.

Krueger DD, Tuffy LP, Papadopoulos T, Brose N (2012) The role of neurexins and neuroligins in the formation, maturation, and function of vertebrate synapses. Current Opinion in neurobiology.

Kulesza RJ, Lukose R, Stevens LV (2011) Malformation of the human superior olive in autistic spectrum disorders. Brain Research 1367:360-371.

Lacaille JC, Mueller AL, Kunkel DD, Schwartzkroin PA (1987) Local circuit interactions between oriens/alveus interneurons and CA1 pyramidal cells in hippocampal slices: electrophysiology and morphology. J Neurosci 7:1979-1993.

Landis DM (1988) Membrane and cytoplasmic structure at synaptic junctions in the mammalian central nervous system. J Electron Microsc Tech 10:129-151.

Landrigan PJ (2010) What causes autism? Exploring the environmental contribution. Curr Opin Pediatr 22:219-225.

Laumonnier F, Bonnet-Brilhault F, Gomot M, Blanc R, David A, Moizard M-P, Raynaud M, Ronce N, Lemonnier E, Calvas P, Laudier B, Chelly J, Fryns J-P, Ropers H-H, Hamel BCJ, Andres C, Barthélémy C, Moraine C, Briault S (2004) X-linked mental retardation and autism are associated with a mutation in the NLGN4 gene, a member of the neuroligin family. Am J Hum Genet 74:552-557.

Levinson JN, El-Husseini A (2005) Building excitatory and inhibitory synapses: balancing neuroligin partnerships. Neuron 48:171-174.

Levinson JN, Li R, Kang R, Moukhles H, El-Husseini A, Bamji SX (2010) Postsynaptic scaffolding molecules modulate the localization of neuroligins. Neuroscience 165:782793.

Levy RB, Aoki C (2002) Alpha7 nicotinic acetylcholine receptors occur at postsynaptic 
densities of AMPA receptor-positive and -negative excitatory synapses in rat sensory cortex. Journal of Neuroscience 22:5001-5015.

Liguz-Lecznar M, Skangiel-Kramska J (2007) Vesicular glutamate transporters VGLUT1 and VGLUT2 in the developing mouse barrel cortex. International Journal of Developmental Neuroscience 25:107-114.

Lisman JE, Idiart MA (1995) Storage of 7 +/- 2 short-term memories in oscillatory subcycles. Science 267:1512-1515.

Lu CB, Jefferys JGR, Toescu EC, Vreugdenhil M (2011) In vitro hippocampal gamma oscillation power as an index of in vivo CA3 gamma oscillation strength and spatial reference memory. Neurobiol Learn Mem 95:221-230.

Luscher B, Fuchs T, Kilpatrick CL (2011) GABAA receptor trafficking-mediated plasticity of inhibitory synapses. Neuron 70:385-409.

Lynch JW (2008) Native glycine receptor subtypes and their physiological roles. Neuropharmacology 56:303-309.

Maccaferri G, Roberts JD, Szucs P, Cottingham CA, Somogyi P (2000) Cell surface domain specific postsynaptic currents evoked by identified GABAergic neurones in rat hippocampus in vitro. J Physiol (Lond) 524 Pt 1:91-116.

Mann EO, Mody I (2010) Control of hippocampal gamma oscillation frequency by tonic inhibition and excitation of interneurons. Nature Neuroscience 13:205-212.

Mann EO, Paulsen O (2007) Role of GABAergic inhibition in hippocampal network oscillations. TRENDS in Neurosciences 30:343-349.

Mann EO, Radcliffe CA, Paulsen O (2005a) Hippocampal gamma-frequency oscillations: from interneurones to pyramidal cells, and back. J Physiol (Lond) 562:55-63.

Mann EO, Suckling JM, Hájos N, Greenfield SA, Paulsen O (2005b) Perisomatic feedback inhibition underlies cholinergically induced fast network oscillations in the rat hippocampus in vitro. Neuron 45:105-117.

Marsicano G, Lutz B (1999) Expression of the cannabinoid receptor CB1 in distinct neuronal subpopulations in the adult mouse forebrain. Eur J Neurosci 11:4213-4225.

Maximov A, Sudhof TC, Bezprozvanny I (1999) Association of neuronal calcium channels with modular adaptor proteins. J Biol Chem 274:24453-24456.

McCormick DA, Prince DA (1986) Mechanisms of action of acetylcholine in the guinea-pig cerebral cortex in vitro. J Physiol (Lond) 375:169-194.

Metherate R, Cox CL, Ashe JH (1992) Cellular bases of neocortical activation: modulation of neural oscillations by the nucleus basalis and endogenous acetylcholine. J Neurosci 12:4701-4711.

Meyer G, Kirsch J, Betz H, Langosch D (1995) Identification of a gephyrin binding motif on the glycine receptor beta subunit. Neuron 15:563-572. 
Meyer G, Varoqueaux F, Neeb A, Oschlies M, Brose N (2004) The complexity of PDZ domain-mediated interactions at glutamatergic synapses: a case study on neuroligin. Neuropharmacology 47:724-733.

Miles R, Tóth K, Gulyás AI, Hájos N, Freund TF (1996) Differences between somatic and dendritic inhibition in the hippocampus. Neuron 16:815-823.

Missler M, Sudhof TC (1998) Neurexins: three genes and 1001 products. Trends Genet $14: 20-26$.

Missler M, Sudhof TC, Biederer T (2012) Synaptic Cell Adhesion. Cold Spring Harbor Perspectives in Biology.

Missler M, Zhang W, Rohlmann A, Kattenstroth G, Hammer RE, Gottmann K, Sudhof TC (2003) Alpha-neurexins couple Ca2+ channels to synaptic vesicle exocytosis. Nature 423:939-948.

Mody I, Pearce RA (2004) Diversity of inhibitory neurotransmission through GABA(A) receptors. TRENDS in Neurosciences 27:569-575.

Mondin M, Labrousse V, Hosy E, Heine M, Tessier B, Levet F, Poujol C, Blanchet C, Choquet D, Thoumine O (2011) Neurexin-neuroligin adhesions capture surface-diffusing AMPA receptors through PSD-95 scaffolds. Journal of Neuroscience 31:13500-13515.

Moon IS, Apperson ML, Kennedy MB (1994) The major tyrosine-phosphorylated protein in the postsynaptic density fraction is N-methyl-D-aspartate receptor subunit 2B. Proc Natl Acad Sci USA 91:3954-3958.

Murcia CL, Gulden F, Herrup K (2005) A question of balance: a proposal for new mouse models of autism. Int J Dev Neurosci 23:265-275.

Murphy CM, Deeley Q, Daly EM, Ecker C, O'Brien FM, Hallahan B, Loth E, Toal F, Reed S, Hales S, Robertson DM, Craig MC, Mullins D, Barker GJ, Lavender T, Johnston P, Murphy KC, Murphy DG (2012) Anatomy and aging of the amygdala and hippocampus in autism spectrum disorder: an in vivo magnetic resonance imaging study of Asperger syndrome. Autism Res 5:3-12.

Okabe S (2007) Molecular anatomy of the postsynaptic density. Mol Cell Neurosci 34:503518.

Panzanelli P, Gunn BG, Schlatter MC, Benke D, Tyagarajan SK, Scheiffele P, Belelli D, Lambert JJ, Rudolph U, FRITSCHY J-M (2011) Distinct mechanisms regulate GABAA receptor and gephyrin clustering at perisomatic and axo-axonic synapses on CA1 pyramidal cells. J Physiol (Lond) 589:4959-4980.

Papadopoulos T, Eulenburg V, Reddy-Alla S, Mansuy IM, Li Y, Betz H (2008) Collybistin is required for both the formation and maintenance of GABAergic postsynapses in the hippocampus. Mol Cell Neurosci 39:161-169.

Persico AM, Bourgeron T (2006) Searching for ways out of the autism maze: genetic, epigenetic and environmental clues. TRENDS in Neurosciences 29:349-358. 
Porter JT, Johnson CK, Agmon A (2001) Diverse types of interneurons generate thalamusevoked feedforward inhibition in the mouse barrel cortex. Journal of Neuroscience 21:2699-2710.

Poulopoulos A, Aramuni G, Meyer G, Soykan T, Hoon M, Papadopoulos T, Zhang M, Paarmann I, Fuchs C, Harvey K, Jedlicka P, Schwarzacher SW, Betz H, Harvey RJ, Brose N, Zhang W, Varoqueaux F (2009) Neuroligin 2 drives postsynaptic assembly at perisomatic inhibitory synapses through gephyrin and collybistin. Neuron 63:628-642.

Prange O, Wong TP, Gerrow K, Wang YT, El-Husseini A (2004) A balance between excitatory and inhibitory synapses is controlled by PSD-95 and neuroligin. Proc Natl Acad Sci USA 101:13915-13920.

Radyushkin K, Hammerschmidt K, Boretius S, Varoqueaux F, El-Kordi A, Ronnenberg A, Winter D, Frahm J, Fischer J, Brose N, Ehrenreich H (2009) Neuroligin-3-deficient mice: model of a monogenic heritable form of autism with an olfactory deficit. Genes Brain Behav 8:416-425.

Reissner C, Klose M, Fairless R, Missler M (2008) Mutational analysis of the neurexin/neuroligin complex reveals essential and regulatory components. Proc Natl Acad Sci USA 105:15124-15129.

Remondes M, Schuman EM (2002) Direct cortical input modulates plasticity and spiking in CA1 pyramidal neurons. Nature 416:736-740.

Rubenstein JLR, Merzenich MM (2003) Model of autism: increased ratio of excitation/inhibition in key neural systems. Genes Brain Behav 2:255-267.

Rudenko G, Nguyen T, Chelliah Y, Sudhof TC, Deisenhofer J (1999) The structure of the ligand-binding domain of neurexin Ibeta: regulation of LNS domain function by alternative splicing. Cell 99:93-101.

Sagné C, Mestikawy El S, Isambert MF, Hamon M, Henry JP, Giros B, Gasnier B (1997) Cloning of a functional vesicular GABA and glycine transporter by screening of genome databases. FEBS Lett 417:177-183.

Sankaranarayanan S, Atluri PP, Ryan TA (2003) Actin has a molecular scaffolding, not propulsive, role in presynaptic function. Nature Neuroscience 6:127-135.

Sato K, Zhang JH, Saika T, Sato M, Tada K, Tohyama M (1991) Localization of glycine receptor alpha 1 subunit mRNA-containing neurons in the rat brain: an analysis using in situ hybridization histochemistry. NSC 43:381-395.

Scheiffele P, Fan J, Choih J, Fetter R, Serafini T (2000) Neuroligin expressed in nonneuronal cells triggers presynaptic development in contacting axons. Cell 101:657-669.

Schneider Gasser EM, Straub CJ, Panzanelli P, Weinmann O, Sassoè-Pognetto M, FRITSCHY J-M (2006) Immunofluorescence in brain sections: simultaneous detection of presynaptic and postsynaptic proteins in identified neurons. Nat Protoc 1:1887-1897.

Schubert D, Kötter R, Zilles K, Luhmann HJ, Staiger JF (2003) Cell type-specific circuits of cortical layer IV spiny neurons. Journal of Neuroscience 23:2961-2970. 
Schumann CM, Amaral DG (2006) Stereological analysis of amygdala neuron number in autism. Journal of Neuroscience 26:7674-7679.

Schumann CM, Hamstra J, Goodlin-Jones BL, Lotspeich LJ, Kwon H, Buonocore MH, Lammers CR, Reiss AL, Amaral DG (2004) The amygdala is enlarged in children but not adolescents with autism; the hippocampus is enlarged at all ages. Journal of Neuroscience 24:6392-6401.

Sheng M, Hoogenraad CC (2007) The postsynaptic architecture of excitatory synapses: a more quantitative view. Annu Rev Biochem 76:823-847.

Shink E, Smith Y (1995) Differential synaptic innervation of neurons in the internal and external segments of the globus pallidus by the GABA- and glutamate-containing terminals in the squirrel monkey. J Comp Neurol 358:119-141.

Shipman SL, Schnell E, Hirai T, Chen B-S, Roche KW, Nicoll RA (2011) Functional dependence of neuroligin on a new non-PDZ intracellular domain. Nature Neuroscience:1-11.

Sik A, Penttonen M, Ylinen A, Buzsáki G (1995) Hippocampal CA1 interneurons: an in vivo intracellular labeling study. J Neurosci 15:6651-6665.

Sik A, Ylinen A, Penttonen M, Buzsáki G (1994) Inhibitory CA1-CA3-hilar region feedback in the hippocampus. Science 265:1722-1724.

Smith AJ, Owens S, Forsythe ID (2000) Characterisation of inhibitory and excitatory postsynaptic currents of the rat medial superior olive. J Physiol (Lond) 529 Pt 3:681-698.

Somogyi P, Klausberger T (2005) Defined types of cortical interneurone structure space and spike timing in the hippocampus. J Physiol (Lond) 562:9-26.

Song JY, Ichtchenko K, Sudhof TC, Brose N (1999) Neuroligin 1 is a postsynaptic celladhesion molecule of excitatory synapses. Proc Natl Acad Sci USA 96:1100-1105.

Sudhof TC (2004) The synaptic vesicle cycle. Annu Rev Neurosci 27:509-547.

Sudhof TC (2008) Neuroligins and neurexins link synaptic function to cognitive disease. Nature 455:903-911.

Sumita K, Sato Y, Iida J, Kawata A, Hamano M, Hirabayashi S, Ohno K, Peles E, Hata Y (2007) Synaptic scaffolding molecule (S-SCAM) membrane-associated guanylate kinase with inverted organization (MAGI)-2 is associated with cell adhesion molecules at inhibitory synapses in rat hippocampal neurons. J Neurochem 100:154-166.

Tabuchi K, Blundell J, Etherton MR, Hammer RE, Liu X, Powell CM, Sudhof TC (2007) A neuroligin-3 mutation implicated in autism increases inhibitory synaptic transmission in mice. Science 318:71-76.

Tabuchi K, Blundell J, Etherton MR, Hammer RE, Liu X, Powell CM, Sudhof TC (2009) A Neuroligin-3 Mutation Implicated in

Autism Increases Inhibitory Synaptic 
Transmission in Mice. Science:1-7.

Tabuchi K, Sudhof TC (2002) Structure and evolution of neurexin genes: insight into the mechanism of alternative splicing. Genomics 79:849-859.

Taniguchi H, Gollan L, Scholl FG, Mahadomrongkul V, Dobler E, Limthong N, Peck M, Aoki C, Scheiffele P (2007) Silencing of neuroligin function by postsynaptic neurexins. Journal of Neuroscience 27:2815-2824.

Thomas NS, Sharp AJ, Browne CE, Skuse D, Hardie C, Dennis NR (1999) Xp deletions associated with autism in three females. Hum Genet 104:43-48.

Towers SK, Gloveli T, Traub RD, Driver JE, Engel D, Fradley R, Rosahl TW, Maubach K, Buhl EH, Whittington MA (2004) Alpha 5 subunit-containing GABAA receptors affect the dynamic range of mouse hippocampal kainate-induced gamma frequency oscillations in vitro. J Physiol (Lond) 559:721-728.

Traub RD, Bibbig A, Fisahn A, LeBeau FE, Whittington MA, Buhl EH (2000) A model of gamma-frequency network oscillations induced in the rat CA3 region by carbachol in vitro. Eur J Neurosci 12:4093-4106.

Treves A, Rolls ET (1994) Computational analysis of the role of the hippocampus in memory. Hippocampus 4:374-391.

Tukker JJ, Fuentealba P, Hartwich K, Somogyi P, Klausberger T (2007) Cell type-specific tuning of hippocampal interneuron firing during gamma oscillations in vivo. Journal of Neuroscience 27:8184-8189.

van Hooft JA, Giuffrida R, Blatow M, Monyer H (2000) Differential expression of group I metabotropic glutamate receptors in functionally distinct hippocampal interneurons. Journal of Neuroscience 20:3544-3551.

Van Kampen M, Selbach K, Schneider R, Schiegel E, Boess F, Schreiber R (2004) AR-R 17779 improves social recognition in rats by activation of nicotinic alpha7 receptors. Psychopharmacology (Berl) 172:375-383.

Varoqueaux F, Aramuni G, Rawson RL, Mohrmann R, Missler M, Gottmann K, Zhang W, Sudhof TC, Brose N (2006) Neuroligins determine synapse maturation and function. Neuron 51:741-754.

Varoqueaux F, Jamain S, Brose N (2004) Neuroligin 2 is exclusively localized to inhibitory synapses. Eur J Cell Biol 83:449-456.

Vincent JB, Kolozsvari D, Roberts WS, Bolton PF, Gurling HMD, Scherer SW (2004) Mutation screening of X-chromosomal neuroligin genes: no mutations in 196 autism probands. Am J Med Genet B Neuropsychiatr Genet 129B:82-84.

Waldvogel HJ, Baer K, Allen KL, Rees MI, Faull RLM (2007) Glycine receptors in the striatum, globus pallidus, and substantia nigra of the human brain: an immunohistochemical study. J Comp Neurol 502:1012-1029.

Whittington MA, Traub RD, Jefferys JG (1995) Synchronized oscillations in interneuron 
networks driven by metabotropic glutamate receptor activation. Nature 373:612-615.

Wojcik SM, Brose N (2007) Regulation of membrane fusion in synaptic excitation-secretion coupling: speed and accuracy matter. Neuron 55:11-24.

Wojcik SM, Katsurabayashi S, Guillemin I, Friauf E, Rosenmund C, Brose N, Rhee J-S (2006) A shared vesicular carrier allows synaptic corelease of GABA and glycine. Neuron 50:575-587.

Yan J, Oliveira G, Coutinho A, Yang C, Feng J, Katz C, Sram J, Bockholt A, Jones IR, Craddock N, Cook EH, Vicente A, Sommer SS (2005) Analysis of the neuroligin 3 and 4 genes in autism and other neuropsychiatric patients. Mol Psychiatry 10:329-332.

Yeckel MF, Berger TW (1990) Feedforward excitation of the hippocampus by afferents from the entorhinal cortex: redefinition of the role of the trisynaptic pathway. Proc Natl Acad Sci USA 87:5832-5836.

Yeckel MF, Berger TW (1995) Monosynaptic excitation of hippocampal CA1 pyramidal cells by afferents from the entorhinal cortex. Hippocampus 5:108-114.

Young JW, Crawford N, Kelly JS, Kerr LE, Marston HM, Spratt C, Finlayson K, Sharkey J (2007) Impaired attention is central to the cognitive deficits observed in alpha 7 deficient mice. Eur Neuropsychopharmacol 17:145-155.

Zhang C, Milunsky JM, Newton S, Ko J, Zhao G, Maher TA, Tager-Flusberg H, Bolliger MF, Carter AS, Boucard AA, Powell CM, Sudhof TC (2009) A neuroligin-4 missense mutation associated with autism impairs neuroligin-4 folding and endoplasmic reticulum export. Journal of Neuroscience 29:10843-10854.

Zhu XO, Waite PM (1998) Cholinergic depletion reduces plasticity of barrel field cortex. Cereb Cortex 8:63-72 


\section{Curriculum Vitae}

\section{HAMMER Matthieu}

E-Mail: hammer.matthieu@gmail.com

Date of Birth: January $2^{\text {nd }}, 1983$

\section{ACADEMIC BACKGROUND}

2009-2012
Max-Planck Institute of Experimental Medicine,
Göttingen, Germany

2006-2008

University Louis Pasteur,

Strasbourg, France

\section{3-2006}

University Louis Pasteur,

Strasbourg, France

\section{1-2003}

University Louis Pasteur,

Strasbourg, France

2001

André Maurois Highschool Bischwiller
PhD Student

Master in Science degree

"Cellular and Integrative Neuroscience"

Bachelor in Science degree

"Physiology and Cellular Biology"

First year of the first cycle of Medicine

Scientific Baccalauréat

\section{PROFESSIONAL EXPERIENCES}

January 2009 to June 2012

Department of Molecular Neurobiology, Max Planck Institute for Experimental Medicine, Göttingen, Germany

\section{January to July 2008}

'Institut des Neurosciences

Cellulaires et Intégratives'

Strasbourg, France

\section{Summer 2007}

'Institut des Neurosciences

Cellulaires et Intégratives'

Strasbourg, France

\section{Fall 2007}

University Louis Pasteur,

Strasbourg, France
Doctoral candidate, under the supervision of Prof. Nils Brose and Dr. Frédérique Varoqueaux

\section{Master 2 training,}

Supervised by Dr Matilde Cordero-Erausquin "Characterization of the cholinergic neurons in mice dorsal spinal cord"

\author{
Voluntary Training \\ Supervised by Dr Pierre Veinante
}

\author{
Master 1 Training \\ Supervised by Dr Pierre VEINANTE and Dr Didier \\ PINAULT \\ "Physiology and Pathologies of the thalamo-cortical system"
}




\section{List of Publications}

Hoon M, Soykan T, Falkenburger B, Hammer M, Patrizi A, Schmidt K-F, Sassoè-Pognetto M, Löwel S, Moser T, Taschenberger H, Brose N, Varoqueaux F (2011) Neuroligin-4 is localized to glycinergic postsynapses and regulates inhibition in the retina. Proc Natl Acad Sci USA 108:3053-3058.

Mesnage B, Gaillard S, Godin AG, Rodeau J-L, Hammer M, Engelhardt Von J, Wiseman PW, De Koninck Y, Schlichter R, Cordero-Erausquin M (2011) Morphological and functional characterization of cholinergic interneurons in the dorsal horn of the mouse spinal cord. J Comp Neurol 519:3139-3158. 